\title{
Multilevel feedback architecture for adaptive regulation of learning in the insect brain
}

\author{
Claire Eschbach ${ }^{f, 1}$, Akira Fushiki ${ }^{f, 1,2}$, Michael Winding ${ }^{\wedge, 1}$, Casey M. Schneider-Mizell ${ }^{\wedge, 1,3}$, Mei Shao ${ }^{\wedge, 1}$, \\ Rebecca Arruda ${ }^{1}$, Katharina Eichler ${ }^{1,4}$, Javier Valdes-Aleman ${ }^{1}$, Tomoko Ohyama ${ }^{1,5}$, Andreas S. Thum ${ }^{6}$, \\ Bertram Gerber $^{7}$, Richard D. Fetter ${ }^{1,8}$, James W. Truman ${ }^{1,9}$, Ashok Litwin-Kumar ${ }^{c, 2,10}$, Albert Cardona ${ }^{c, 1,11}$, \\ Marta Zlatic ${ }^{c, 1,12}$
}

Modulatory (e.g. dopaminergic) neurons provide "teaching signals" that drive associative learning across the animal kingdom, but the circuits that regulate their activity and compute teaching signals are still poorly understood. We provide the first synaptic-resolution connectome of the circuitry upstream of all modulatory neurons in a brain center for associative learning, the mushroom body (MB) of the Drosophila larva. We discovered afferent pathways from sensory neurons, as well as an unexpected large population of 61 feedback neuron pairs that provide one- and two-step feedback from MB output neurons. The majority of these feedback pathways link distinct memory systems (e.g. aversive and appetitive). We functionally confirmed some of the structural pathways and found that some modulatory neurons compare inhibitory input from their own compartment and excitatory input from compartments of opposite valence, enabling them to compute integrated common-currency predicted values across aversive and appetitive memory systems. This architecture suggests that the MB functions as an interconnected ensemble during learning and that distinct types of previously formed memories can regulate future learning about a stimulus. We developed a model of the circuit constrained by the connectome and by the functional data which revealed that the newly discovered architectural motifs, namely the multilevel feedback architecture and the extensive cross-compartment connections, increase the computational performance and flexibility on learning tasks. Together our study provides the most detailed view to date of a recurrent brain circuit that computes teaching signals and provides insights into the architectural motifs that support reinforcement learning in a biological system.

\section{Introduction}

To behave adaptively in an ever-changing environment, animals must not only be able to learn new associations between conditioned stimuli (CS) and rewards or punishments (in Pavlovian terms, aversive and appetitive unconditioned stimuli, US), but also continuously update previous memories, depending on their relevance and reliability ${ }^{1-14}$. For example, memories can be consolidated into a persistent form and maintained $^{5,6,8,15-17}$, extinguished $e^{5,7,9,11,14,18}$, or expanded and combined into chains of associations (as in higher-order conditioning $\left.{ }^{7,19,20}\right)$. Furthermore, learning can itself be flexible and depends both on present context and past history ${ }^{14,21,22}$. These are fundamental brain functions across the animal kingdom, but the learning algorithms used by brains and their circuit implementations are still unclear.

${ }^{1}$ HHMI Janelia Research Campus, 19700 Helix Dr., Ashburn, VA 20147. ${ }^{2}$ Dept. of Neuroscience \& Neurology, \& Zuckerman Mind Brain Institute, Columbia Univ., New York, USA. ${ }^{3}$ Allen Institute for Brain Science, Seattle, WA, USA ${ }^{4}$ Institute of Neurobiology, Univ. of Puerto Rico Medical Science Campus. ${ }^{5} \mathrm{McGill}$ Univ., Dept. of Biology, Montreal, QC, Canada. ${ }^{6}$ Dept. of Genetics, Institute for Biology, Univ. of Leipzig, Germany. ${ }^{7}$ Abteilung Genetik von Lernen \& Gedächtnis, Leibniz Institut für Neurobiologie; Otto von Guericke Univ. Magdeburg, Institut für Biologie, Verhaltensgenetik, \& Center for Behavioral Brain Sciences, Magdeburg, Germany. ${ }^{8}$ Molecular Biochemistry \& Biophysics, UCSF. ${ }^{9}$ Dept. of Biology, Univ. of Washington. ${ }^{10}$ Dept. of Neuroscience, Center for Theoretical Neuroscience, \& Zuckerman Mind Brain Institute, Columbia Univ., New York, USA. ${ }^{11}$ Dept. of Physiology, Development \& Neuroscience, Univ. of Cambridge. ${ }^{12}$ Dept. of Zoology, Univ. of Cambridge. ${ }^{f}$ co-first authors; ${ }^{\wedge}$ cosecond authors; ${ }^{c}$ corresponding authors: zlaticm@janelia.hhmi.org, cardonaa@janelia.hhmi.org, ak3625@columbia.edu.
Modulatory neurons (e.g. dopaminergic, DANs) convey information about rewards and punishments and provide the so-called teaching signals for updating the valence associated with CS in learning circuits across the animal kingdom (e.g. in the vertebrate basal ganglia, or in the insect mushroom body, MB) ${ }^{12,14,21,23-25}$. In the simplest models of associative learning, learning is driven by correlations between CS and US, and modulatory neuron activity represents just the received $\mathrm{US}^{26,27}$. To account for more complex behavioral phenomena, theories have been developed in which learning can be regulated by previously formed associations and modulatory neuron responses to CS are adaptively modified by prior learning $4,12,21,28-36$. For example, in reinforcement learning, learning is driven by errors between predicted and actual US (so-called prediction errors $)^{21,28-35}$, which are represented by the activity of modulatory neurons. Indeed, responses of many modulatory neurons have been shown to be adaptive, in monkeys ${ }^{21,31,32,34,37}$, rodents ${ }^{12,25,36,38-41}$, and insects $^{13,23,42-44}$, although the extent to which this is the case in insects has been less extensively investigated. Despite recent progress ${ }^{12-14,41,45-49}$, the basic principles by which modulatory neuron activity is adaptively regulated, and what teaching signals they compute and encode, are not well understood.

A prerequisite for the adaptive regulation of modulatory neuron activity is convergence of afferent pathways that convey information about received rewards and punishments ${ }^{12,21}$ with feedback pathways that convey information about previous experience. In the rodent basal ganglia, DANs have indeed been shown to receive feedback from striatum and striatal neurons have been implicated in prediction error computations ${ }^{12,39,50-52}$. In the Drosophila mushroom body (MB), some DANs have 
also been shown to receive direct feedback input from $\mathrm{MB}$ output neurons and specific output neurons have been implicated in memory updating ${ }^{13,14,46-49,53-55}$. However, we still know very little about the nature of these feedback circuits and the way in which they compute features such as predictions and prediction errors. How much input do modulatory neurons receive from afferent vs. feedback pathways? Do these pathways converge on modulatory neurons themselves, or at multiple levels upstream? Distinct types of memories (e.g. aversive and appetitive, short-term and long-term) are often formed in distinct locations, in both flies ${ }^{14,24,56-58}$ and rodents ${ }^{59-61}$, but are there feedback pathways that enable memories of one type to influence the formation of memories of a different type? How are integrated common-currency predictions across aversive and appetitive memory systems computed? How many distinct types of feedback motifs are there and what computational advantages do they offer? Addressing these questions is essential for understanding how learning algorithms are implemented in neural circuits. However, such a comprehensive characterization of feedback pathways requires a synaptic-resolution connectivity map of the complete set of modulatory neurons, their target output neurons, and of all of their pre- and post-synaptic partners, which has previously been out of reach.

Insects, especially their larval stages, have small and compact brains that have recently become amenable to large-scale electron microscopy (EM) circuit mapping ${ }^{47,62}$. Both adult ${ }^{8,14,27,48,63-67}$ and larval ${ }^{47,57,58,68-70}$ insect stages possess a brain center essential for associative learning, the MB. The MB contains parallel fiber neurons called Kenyon Cells (KCs) that sparsely encode $\mathrm{CS}^{66,71-74}$; MB modulatory neurons (collectively called MBINs) that provide the teaching signals for updating the valence associated with $\mathrm{CS}^{13,14,42,46,47,55-58,74-84}$; and $\mathrm{MB}$ output neurons (MBONs) whose activity represents learnt valences of stimuli ${ }^{14,58,66,85-93}$. Most modulatory neurons are dopaminergic (we called this subset DANs, adding a letter that indicates their target compartment in the MB, e.g. DAN-g1), some are octopaminergic (OANs, e.g. OAN-g1), and some have unidentified neurotransmitters (so we refer to this subset by their generic name, e.g. MBIN-e1). Modulatory neurons and MBONs project axon terminals and dendrites, respectively, onto the $\mathrm{KC}$ axons in a tiled manner, defining MB compartments, in both adult ${ }^{14,45,48,66,75,94}$ and larval ${ }^{47,58,95}$ Drosophila. In adult Drosophila, it has been shown that co-activation of $\mathrm{KCs}$ and DANs reduces the strength of the KC-MBON synapse in that compartment ${ }^{14,55,66,85,90,91,96,97}$. Different compartments have been implicated in the formation of distinct types of memories, for example aversive and appetitive, or shortand long-term $14,24,56-58,66,74,76,81,83,84,89-91,98-100$. However, the extent to which learning in a specific compartment is regulated by the output from its own compartment or from other compartments is still unclear. A few direct anatomical connections from MBONs to modulatory neurons have been identified ${ }^{13,14,45-49,53,54}$, but possible indirect connections via intermediate feedback neurons have not been investigated. In total, despite a good understanding of the structure and function of the core components of the $\mathrm{MB}^{14,47,48,58}$, the circuits presynaptic to modulatory neurons that regulate their activity have re- mained largely uncharacterized, in both adult and larval Drosophila.

We therefore reconstructed all neurons upstream of all modulatory neurons in an EM volume that spans the entire nervous system of a 1st instar Drosophila larva, in which we had previously reconstructed all the core components of the MB (including $145 \mathrm{KCs}, 48 \mathrm{MBONs}$ and 28 modulatory neurons $)^{47}$. Working in the same EM volume enabled us to not only identify all the neuron types upstream of the modulatory neurons, but also to precisely determine which MBONs they receive input from. The present EM reconstruction effort was even larger than reconstructing the core components, because of the large number of neuron types presynaptic to modulatory neurons. We reconstructed a total of 431 previously unknown neurons, of which 102 pairs of homologous neurons (i.e. present in each brain hemisphere) make at least 3 synaptic connections onto the modulatory neurons. We also determined which individual modulatory neurons are activated by punishments and reconstructed their afferent US pathways from nociceptive and mechanosensory neurons. We characterized the neurotransmitter profiles of some of the neurons in the network and functionally confirmed some of the identified structural connections. Finally, we developed a model of the circuit constrained by the connectome, the neurotransmitter data, and the functional data and used it to explore the computational advantages offered by the newly discovered architectural motifs for performing distinct learning tasks.

Surprisingly, we found that the majority of neuron types (61 out of 102) presynaptic to modulatory neurons provide one-step or two-step feedback from MBONs. Furthermore, modulatory neurons received extensive input not only from MBONs in their own compartment, but also from many other compartments. In our model the multilevel and cross-compartment feedback architecture improves computational performance on learning tasks that rely on the adaptation of modulatory neuron responses. These pathways may therefore form the neural architecture that permits previously formed associations to instruct future learning, a critical computation in brain circuits that implement reinforcement learning algorithms.

\section{Results}

\section{Larval MB modulatory neurons for aversive and appetitive memory formation}

To more easily interpret the circuitry for regulating modulatory neuron activity and to better constrain models of the circuit, we explored the functional diversity of larval modulatory neurons and identified individual compartments of the larval MB whose modulatory neuron activation paired with odor can evoke aversive or appetitive memory. Previous studies have already shown that pairing of an odor with activation of all, or individual DANs, which target the MB medial lobe (ML), induces appetitive memory ${ }^{47,57,58}$. Another study has shown that pairing an odor with the activation of all DANs that target the vertical lobe (VL), the lateral appendix (LA), and the peduncle $(\mathrm{P})$ (with a broadly-expressing TH-GAL4 driver line) induces aversive memory ${ }^{56}$. To disentangle the role of individual modulatory neuron types in aversive learning, we 
a

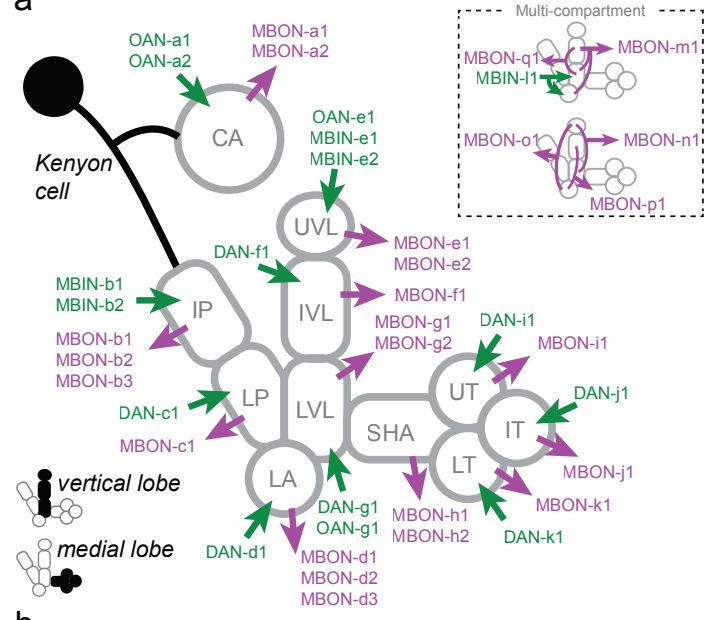

b

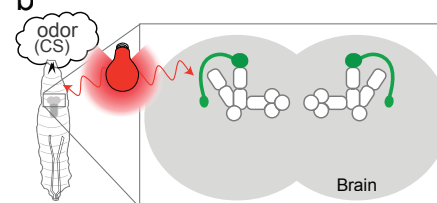

Modulatory neuron > CsChrimson
C

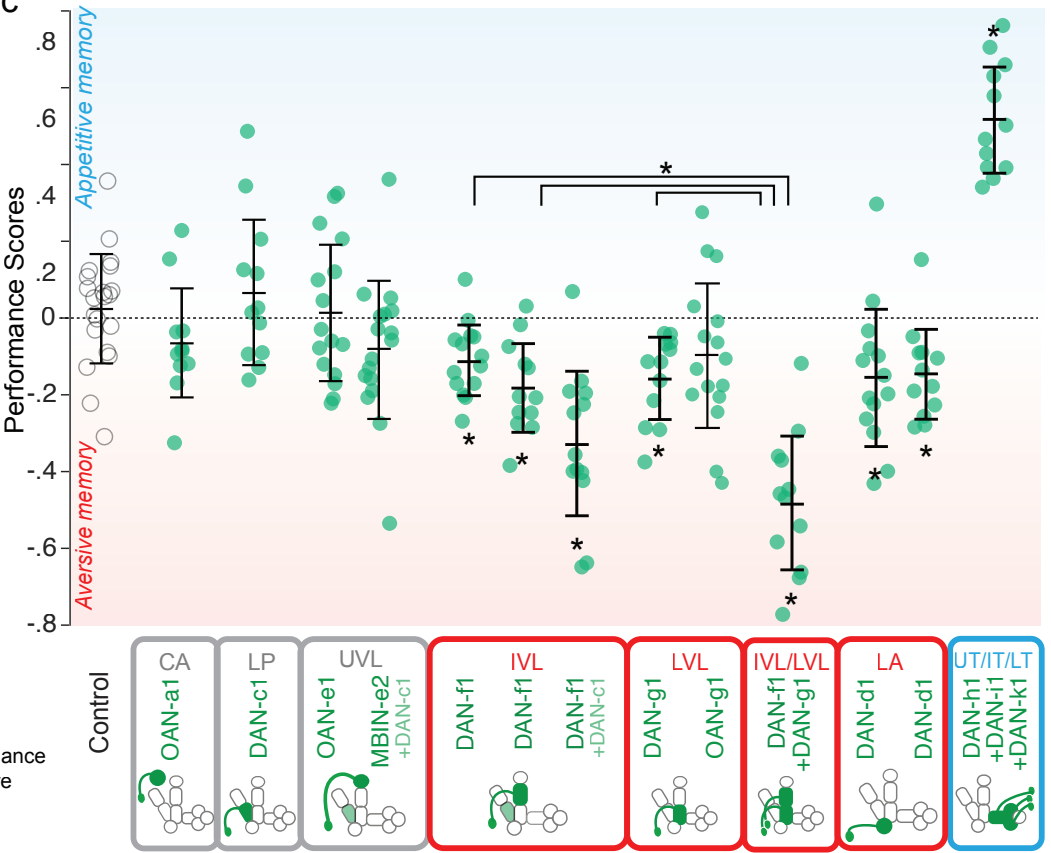

d

Mechanosensory Ch

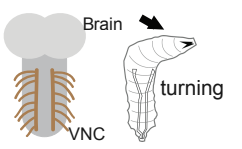

Nociceptive MD IV

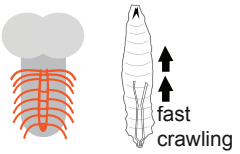

Multisensory Basins

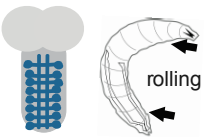

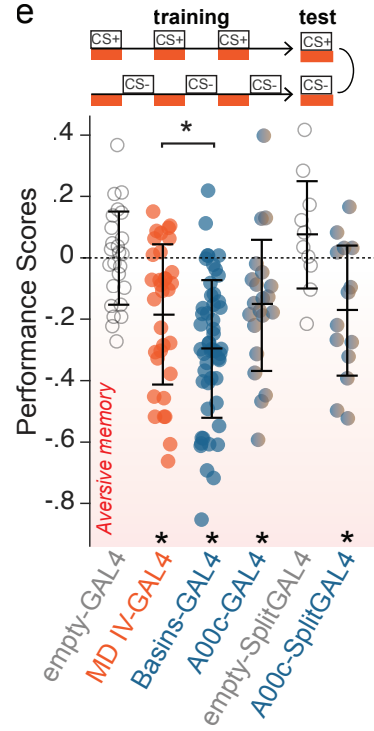

f
Modulatory neuron > GCaMP6f

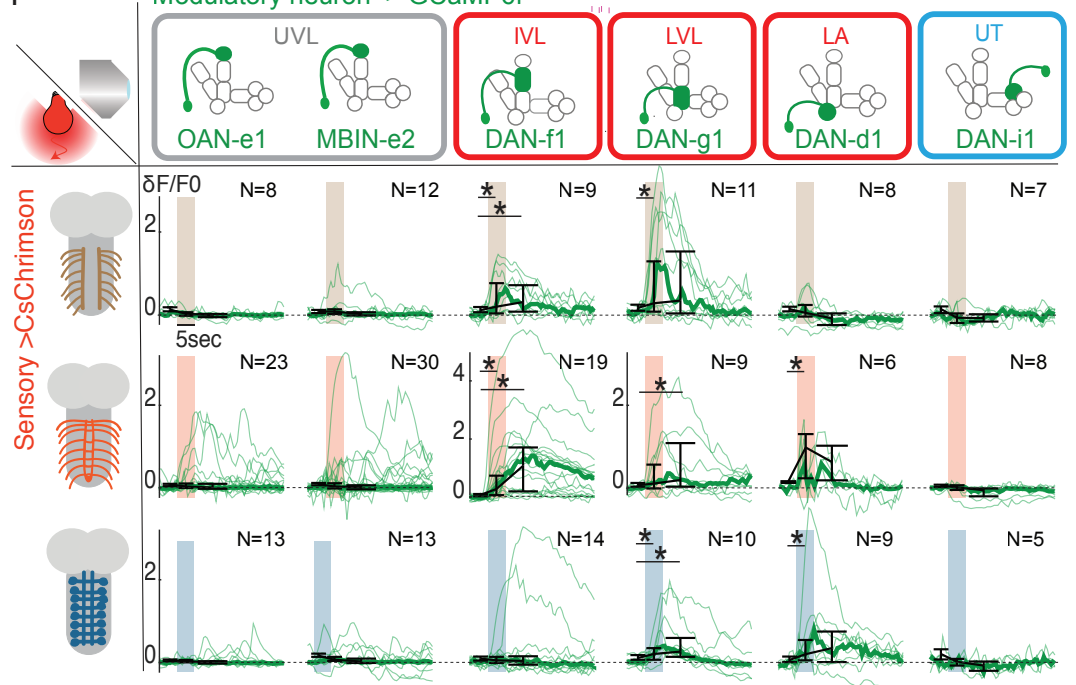

Figure 1 || Individual vertical lobe DANs can induce aversive memory and represent different kinds of punishments in Drosophila larva.

a Schematic diagram of the larval mushroom body (MB) compartments with an example Kenyon cell (black), all Mushroom Body Output Neurons (MBONs, purple) and modulatory neurons (green), named based on neurotransmitter expression and compartmental localisation: dopaminergic neurons (DANs), octopaminergic (OANs), or unknown (MBINs). CA: calyx; LP, IP: lower and intermediate peduncle; LA: lateral appendix; VL: vertical lobe; UVL, IVL, LVL: upper, intermediate and lower vertical lobe; SHA, UT, IT, LT: shaft, upper, intermediate and lower toe of the medial lobe.

b Schematic diagram of the one-odor associative memory optogenetic training protocol consisting of three training trails (3 min each). Larvae from "paired" groups are trained to associate an odor (ethyl acetate) with optogenetic activation (red light) of a pair of modulatory neurons expressing CsChrimson. Larvae from "unpaired" groups undergo bouts of odor presentation intercalated with bouts of modulatory neuron activation. A preference index $(P I)$ was computed for each group: $P I=[N$ (larvae on the odor side) $-N($ larvae on the no-odor side)] / N(total). The learning performance score $(L P S)$ was then computed: $L P S=[P I($ paired $)-P I($ unpaired $)] / 2$. Positive learning performance scores result from a higher fraction of larvae on the odor side in the paired group than in the unpaired group, indicating appetitive memory; negative learning performance scores indicate aversive memory.

c Activation of vertical or medial lobe DANs induces memories of opposite valence. Figure shows learning performance scores obtained with CsChrimson-mediated activation of one or a few pair(s) of modulatory neurons using Split-GAL4 in a one-odor associative memory protocol: OAN-a1 (SS24765, n=11), DAN-c1 (SS02160, $\mathrm{n}=12)$, OAN-e1 $(S S 01958, \mathrm{n}=16)$, combined MBIN-e2 and DANc1 (SS01702, n=18), DAN-f1 $(M B 145 B, \mathrm{n}=14$ and SS02180, $\mathrm{n}=12)$, combined DAN-f1 and DAN-c1 $(M B 065 B, \mathrm{n}=14)$, DAN-g1 (SS01716, $\mathrm{n}=12)$, OAN-g1 (SS04268, $\mathrm{n}=16)$, combined DAN-f1 and DAN-g1 (MB054B, $\mathrm{n}=12)$, DAN-d1 $(M B 143 B, \mathrm{n}=14$ and $M B 328 B$, $\mathrm{n}=12$ ), combined DAN-h1, DAN-i1, DAN-k1 (SS01948, n=12), control $(w ; a t t P 40 ; a t t P 2, n=21)$. Pairing of odor with optogenetic activation of individual DAN-f1, -g1 and -d1 in VL or LA induces aversive memory. In contrast, pairing of odor with the combined optogenetic activation of DAN-h1, -i1 and -k1 in the ML induces appetitive memory. MB compartments for aversive memory (negative performance scores) are outlined in red; compartments for appetitive memory (positive performance scores) are outlined in blue.

Each data point represents the learning performance score for one reciprocal experiment involving one "paired" and one "unpaired" group. Mean and standard deviations are plotted. *: significant difference from the scores obtained in the control group (open circles, Mann-Whitney U test P-values compared to threshold of 0.05 adjusted with a Holm-Bonferroni correction for multiple comparisons). d Different types of somatosensory neurons induce distinct types of innate escape responses. Optogenetic or thermogenetic activation of mechanosensory neurons (chordotonal, Ch, brown), nociceptive multidendritic class IV neurons (MD IV, orange), or multisensory interneurons (Basins, blue) induces turning, fast forward crawling or rolling, respectively (Ohyama et al. 2013, 2015, Jovanic et al. 2016). e Optogenetic activation of nociceptive MD IV (ppk1.9-GAL4, $\mathrm{n}=33$ ), Basins (GMR72F11-GAL4, $\mathrm{n}=52$ ), or the ascending neuron A00c (postsynaptic to Basins, GMR71A10-GAL4,ppk-GAL80,repo(Continues into the next page.) 
(Continues from prior page, Figure 1.)

GAL80, n=21 or SS00883-Split-GAL4, n=14) induces aversive memory when paired with odor (ethyl acetate). Learning performance scores were computed as described in $\mathbf{b}$. The larvae were tested under constant optogenetic activation, as depicted in the schematic diagram, to ensure the expression of aversive memory (See Extended Data Fig. 2 for details). Mean and standard deviations are plotted, as well as significant differences from the scores of the respective control group $(w ; ; a t t P 2, \mathrm{n}=25$ or $w$;attP40;attP2, $\mathrm{n}=10$, according to the driver line, open circles), in the same way as in c.

f DANs whose activation paired with odor induces aversive memory respond consistently to optogenetic activation of somatosensory neurons. DAN-f1 preferentially responds to $\mathrm{Ch}$ and MD IV stimulations, DAN-d1 to MD IV and Basins stimulations, and DAN-g1 responds to all three types of stimulation. Plots show calcium transients

generated Split-GAL4 lines ${ }^{101,102}$ that drive expression selectively in one or two modulatory neurons per hemisphere (Fig. 1a, Extended Data Fig. 1, Supplementary Table 1). We then paired an odor (CS) with Chrimson-mediated optogenetic activation $^{103}$ of these modulatory neurons in a three-trial, one-odor, associative memory paradigm (Fig. 1b). Because we tested larvae immediately after the last training trial, and less than $20 \mathrm{~min}$ after the first training trial, we assume the test reveals mainly short-term memory $^{70}$.

We found that pairing an odor with the activation of DAN-f1 (projecting to the intermediate vertical lobe, IVL), DAN-g1 (projecting to lower vertical lobe, LVL), or DAN-d1 (projecting to LA) established aversive memory (Fig. 1c and Extended Data Fig. 2a). In contrast, and as previously reported $47,57,58$, pairing an odor with the activation of DANs that project to the ML (DAN-h1, DAN-i1, and DAN-k1) led to the formation of an appetitive memory (Fig. 1c and Extended Data Fig. 2a). Thus, similar to findings in the adult fly ${ }^{14,24,66,74,76-78,84,104,105}$, larval DANs that innervate distinct lobes are functionally distinct from each other, in that their activation signals opposite valences. Activation of larval PAM-cluster DANs that innervate the ML signals positive valence, whereas activation of larval DL-cluster ${ }^{95}$ DANs that innervate the VL and the LA signals negative valence. Our results also suggest, in accordance with other studies ${ }^{7,8,13,106}$, that presenting an odor unpaired with the activation of some of these DANs induces memory of opposite valence to the paired presentation (Extended Data Fig. 2b).

We found that pairing of an odor with the activation of DAN-c1 that projects to lower peduncle (LP) induced neither appetitive nor aversive memory (Fig. 1c and Extended Data Fig. 2a). Similarly, no memory was induced by pairing an odor with activation of any OANs, or of MBIN-e2 (which was immunonegative for dopamine, octopamine, acetlylcholine, GABA, and glutamate ${ }^{47}$ ). Thus, DAN-c1, OANs, and MBIN-e2 appear to be functionally distinct from the lobe DANs (with a possible caveat that the GAL4 lines for these neurons may be weaker than the ones for lobe DANs). What role, if any, these neurons play in learning remains to be uncovered in the larva (for some roles of the OAN system see ${ }^{42,56,79,107}$ ). In any case our analysis has revealed at least three functionally distinct classes of compartments in the larval MB: ML compartments whose DANs can induce appetitive memory when their activation is paired with odor; LA, LVL and IVL compartments whose DANs can induce aversive in selected modulatory neurons evoked by optogenetic activation of Ch (iav-LexA>LexAop-CsChrimson, top row), MD IV ( ppk1.9LexA>LexAop-CsChrimson, middle row) and Basin (GMR72F11LexA $>$ LexAop-CsChrimson, bottom row) neurons. GCaMP6f was expressed in OAN-e1 (SS01958), MBIN-e1 (SS01702), DAN-f1 $(M B 145 b)$, DAN-g1 (SS01716), DAN-d1 (MB143b), or DAN-i1 (SS00864) using Split-GAL4. In each plot, thin lines are the averaged responses for one brain, from 3 repeats (triplet trial); thick lines are the median across all animals tested. Black plots indicate the median peak $\delta \mathrm{F} / \mathrm{F} 0$ of the individual curves in the following time windows: 1 sec before, 1 sec during, and 2 sec following the stimulation. Errorbars show the 25 th and 75 th percentile of peak $\delta \mathrm{F} / \mathrm{F} 0$ of the individual curves. *, $\mathrm{P}<0.05$ Mann-Whitney U-test. Outline color corresponds to the type of memory induced by the neuron, as described in $\mathbf{c}$

memory when their activation is paired with odor; and others whose modulatory neurons were not sufficient to induce memory (Fig. 1c).

\section{Punishment encoding across larval MB modula- tory neurons}

Next, we asked whether there is any functional diversity within the population of VL/LA DANs whose activation signals punishment. In principle, multiple DANs that project to distinct VL/LA compartments could represent a functionally uniform population and redundantly signal any type of aversive US, as proposed for DANs that signal reward in the mammalian ventral tegmental area ${ }^{12,40}$. Alternatively, distinct DANs could signal distinct types of aversive US, as proposed for DANs that signal rewards in Drosophila larva and adult $58,74,78,79,82,83,108,109$. In the adult, the same DANs can convey the teaching signals for different aversive stimuli ${ }^{77,81,104,110-112}$, and respond to multiple aversive stimuli ${ }^{75,110-113}$, but some DANs also appear to be preferentially tuned to some aversive stimuli, but not others ${ }^{75,111,112}$. The extent to which individual DANs that signal aversive stimuli are functionally diverse and whether and how punishment quality or punishment salience may be encoded by DANs in Drosophila are therefore open questions.

Larvae sense multiple types of innately aversive somatosensory stimuli that evoke distinct types of innate responses ${ }^{62,114-124}$ (Fig. 1d). Vibration or optogenetic activation of the vibration-sensing mechanosensory neurons evokes hunching (startle) and turning (avoidance) $^{62,114,119,121,123,124}$. Optogenetic activation of nociceptive neurons evokes a more vigorous escape response: fast crawling $62,116,119,123$. Wasp attack that stimulates both nociceptive and mechanosensory neurons, or optogenetic activation of Basin interneurons that integrate mechanosensory and nociceptive inputs, evokes the most vigorous and fastest escape response: rolling $62,115,119,122,125$. Already the mildest of these punishments, vibration, induces aversive associative memory in an olfactory learning paradigm ${ }^{126}$. Fittingly, we found that the stronger forms of punishment, namely, optogenetic activation of the nociceptive sensory neurons, Basins, or the A00c neurons that are directly downstream of Basins, also induce aversive associative memory when paired with odor (Fig. 1e and Extended Data Fig. 2a). We therefore asked how individual modulatory neurons respond to each type of punishment administered by optogenetic activation of specific somatosensory-related 
a

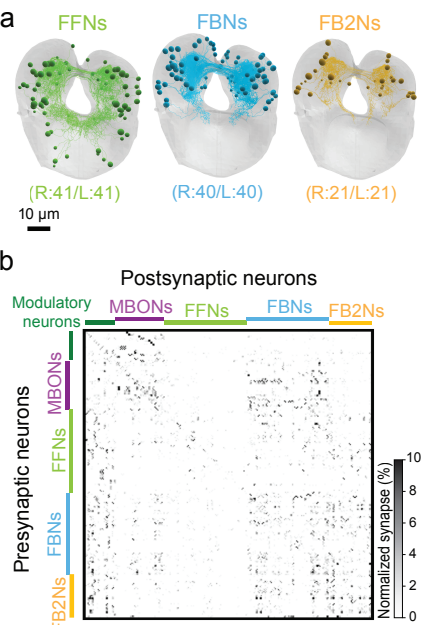

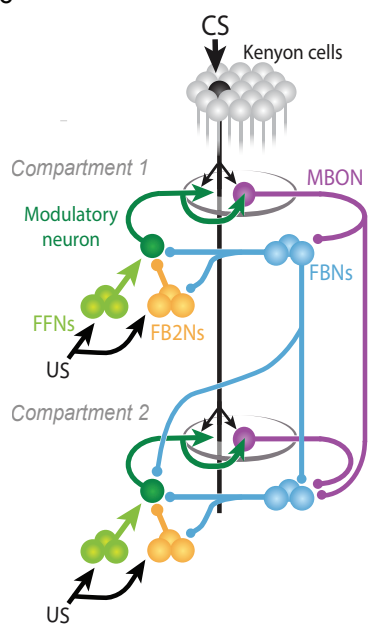

d MBONs FBNs FB2Ns FFN KCs Modulatory neurons Weak connections

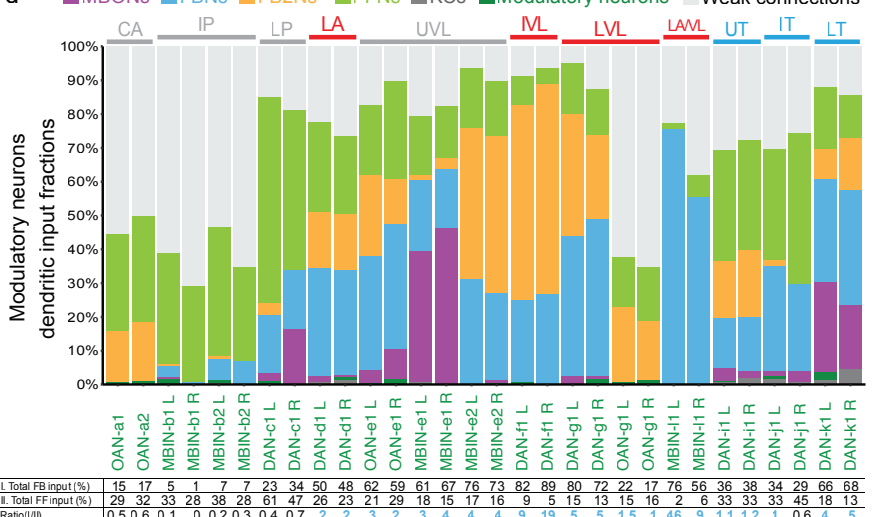

f

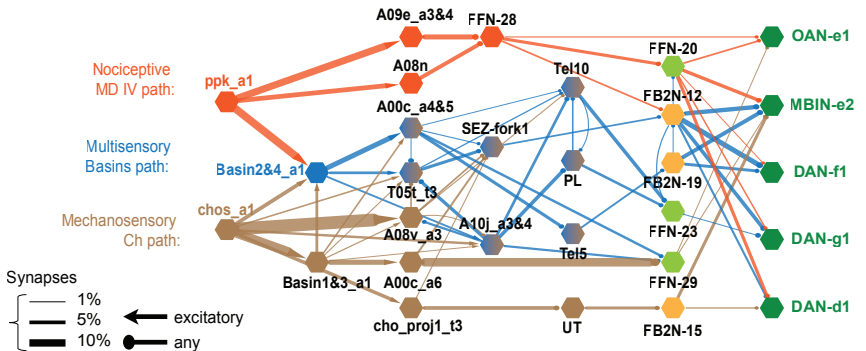
pressed as \% input) each postsynaptic (columns) neuron receives from each presynaptic (rows) neuron in the extended MB circuit, comprising modulatory neurons (green), MBONs (purple), and the different types of pre-modulatory neurons.

The normalization is done by computing the $\%$ of total input a postsynaptic neuron receives from a presynaptic neuron i.e. by dividing the number of synapses from a presynaptic neuron to a postsynaptic neuron by the total number of input synapses of that postsynaptic neuron and multiplying by 100 . The average for left and right homologs is shown. Only reliable connections for which both the left and right homologous connections have at least 3 synapses and their sum is at least 10 are shown.

Note that among the pre-modulatory neurons, FBNs (blue) and FB2Ns (yellow) are more interconnected to each other than to FFNs (light green).

c, Layers of feedback neurons reveal the highly recurrent architecture of the circuits. Figure shows a schematic wiring diagram of the core components of the MB together with the newly discovered components upstream of modulatory neurons and downstream of MBONs. Most modulatory neurons receive one-step and two-step within and cross-compartment feedback.

d Fraction of total dendritic input each modulatory neuron receives from MBONs, FBNs, FB2Ns, FFNs, and from other weakly connected partners (i.e. those that make less than 3 synapses onto a modulatory neuron and less than 10 synapses onto any left-right pair of homologous modulatory neurons). Many modulatory neu-

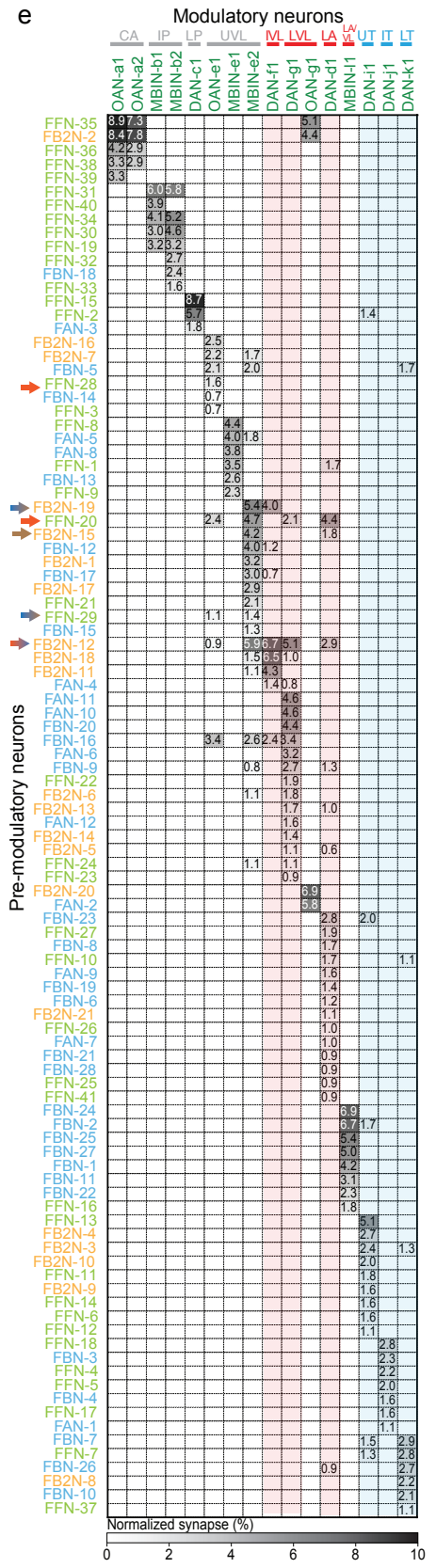

Figure 2 || Comprehensive EM reconstruction of premodulatory neurons reveals a multilayered recurrent architecture for regulating learning.

a-c Comprehensive EM reconstruction of all neurons presynaptic to all modulatory neurons reveals a large previoulsy unknown population of feedback neurons.

a, The newly discovered 102 neuron pairs presynaptic to the modulatory neurons. Of these, the majority (61) relays inputs from mushroom body output neurons (MBONs): 40 first-order feedback neuron pairs (FBNs, light blue) receive direct input from MBONs and provide one-step feedback to modulatory neurons; and 21 second-order feedback neuron pairs (FB2Ns, yellow) receive one-step MBON input via FBNs and provide two-step feedback to modulatory neurons. The remaining 41 are tentatively classified as feedforward neurons (FFNs, light green) that may represent unconditioned stimuli (US). Projections of EM reconstructions of all neurons within each category are shown. The complete set of neurons presynaptic to modulatory neurons amounts to 211 leftright pairs and 5 bilaterally projecting unpaired neurons (not shown). Of these, only 204 neurons (102 pairs) make at least 3 synapses onto any particular modulatory neuron and together as leftright pairs make at least 10 synapses onto any particular left-right pair of homologous modulatory neurons, and are therefore considered reliably and strongly connected.

rons, including most of the DANs, receive more than half of their total dendritic input from the feedback pathways (i.e. from MBONs, FBNs, and FB2Ns), indicating the likely importance of these pathways for governing modulatory neuron activity and thereby learning. The OANs in the calyx (CA), intermediate peduncle (IP) and low vertical lobe (LVL), are drastically different from other modulatory neurons in that they receive a large fraction of input from many weakly connected partners (i.e. neurons that connect to modulatory neurons with very few synapses as explained in a), and relatively little feedback from MBONs. These OANs may therefore encode very different features from the rest of the modulatory neurons. Some DANs extend their dendritic arbors to the KCs which accounts for a small proportion of $\mathrm{KC}$ dendritic input. We also note that $\mathrm{KCs}$ can modulate the output of modulatory neurons through axo-axonic connections (Eichler et al. 2017, Cervantes-Sandoval et al. 2017, Takemura et al. 2017), but the presynaptic modulatory input likely has a very distinct functional role from the dendritic input and we do not consider it further in this study.

Bottom: Table shows percent of inputs onto dendrites of modulatory neurons from (I.) all known feedback types (direct MBON feedback, one-step FBN feedback, and two-step FB2N feedback), (II.) FFNs (likely conveying feedforward input from US pathways), and their ratio. Note that for most modulatory neurons this ratio is greater than 1 (color coded in blue), suggesting feedback input may be at least as important as feedforward input for regulating modulatory neuron activity.

(Continues into the next page.) 
(Continues from prior page, Figure 2.)

e Connectivity matrix showing normalized synaptic input (expressed as $\%$ input, computed as in $\mathbf{b}$ ) each modulatory neuron (columns) receives from each pre-modulatory neuron (rows). Only reliable connections are shown for which both the left and right homologous connections have at least 3 synapses, and their sum is at least 10 . Modulatory neurons are color-coded as in c according to the type of memory they can induce, red for aversive and blue for appetitive). Many pre-modulatory neurons (rows) synapse onto a single modulatory neuron, or onto modulatory neurons of similar function (columns). Functionally distinct modulatory neurons (e.g. DANs whose activation signals positive and negative valence, Fig. 1b) that innervate distinct $\mathrm{MB}$ lobes receive inputs from distinct subsets of pre-modulatory neurons (See also Extended Data Fig. 3). Interestingly, two UVL MBINs (OAN-e1 and MBIN-e2), whose activation paired with odor did not induce memory in our paradigm and that were not significantly activated by fictive punishments share a

neurons, by monitoring their calcium transients (using GCaMP6f ${ }^{127}$ ) (Fig. 1f). We performed these experiments in isolated nervous systems to avoid any movement-related responses $^{55}$.

In each of the VL/LA-DANs whose activation paired with odor induced aversive memory, we found reliable responses to at least two of the three fictive punishment types. Each punishment type evoked reliable and statistically significant responses in at least two out of three VL/LA-DANs (Fig. 1f). Nevertheless, individual VL/LA-DANs differed in terms of which punishment types evoked reliable and statistically significant responses (Fig. 1f). Specifically, DAN-g1 responded non-selectively to all three punishment types, DAN-f1 responded preferentially to mechanosensory and nociceptive neuron activation, and DAN-d1 to nociceptive and Basin neuron activation. Therefore, considering one of these DANs alone would not allow decoding punishment type, but considering all three would. Thus, the three VL/LA DANs could combinatorially encode punishment type or punishment salience.

For comparison, we also tested the response of a few modulatory neurons whose activation paired with odor did not induce aversive memory. As expected, we found that DAN-i1 (projecting to ML) whose activation paired with odor induced appetitive memory was not activated by the fictive punishments (Fig. 1f). Modulatory neurons that project to UVL (OAN-e1 and MBIN-e2) and whose activation paired with odor induced neither aversive nor appetitive memory were not significantly activated by the fictive punishments, although we observed occasional responses to nociceptive neuron activation (Fig. 1f).

\section{Comprehensive EM reconstruction of all input neurons to larval MB modulatory neurons}

To provide a basis for understanding how the activity and function of modulatory neurons is regulated we sought to comprehensively identify all the neurons presynaptic to them. We have previously reconstructed all of the $\mathrm{KCs}$, olfactory projection neurons (PNs), MBONs, and modulatory neurons in an EM volume of a 1st instar larval nervous system ${ }^{47}$. Here we systematically reconstructed all neurons presynaptic to all modulatory neurons in the same EM volume (Fig. 2a-e). We identified 213 left-right homologous pairs and 5 unpaired neurons presynaptic to modulatory neurons (that we collectively higher fraction of their input with the VL/LA DANs than with other modulatory neurons. This raises the possibility that the UVL modulatory neurons map be recruited by similar stimuli to the VL/LA DANs, but only in specific circumstances.

f US pathways converge with feedback pathways from MBONs at multiple levels: at the modulatory neurons themselves, and at FB2Ns. The EM connectivity map shows the shortest identified pathways from distinct types of US pathways from somatosensory neurons to VL modulatory neurons. US pathways target both FFNs and FB2Ns. The hexagonal shape denotes a group of left-right homolog neurons. Connections with less than 10 synapses are not included (see Supplemental Adjacency Matrix for complete connections). Orange, Nociceptive MD IV specific pathway; brown, Mechanosensory Ch specific pathway; blue, multimodal Basins 2\&4 pathway. Thickness of the arrow is proportional to normalized synaptic input that a postsynaptic neuron type receives from a presynaptic neuron type at the source of the arrow, defined as in $\mathbf{b}$.

called pre-modulatory neurons). Out of these, we consider 102 homologous pairs to be reliably connected, since both the left and right homologous connections have at least 3 synapses and their sum is at least 10 (thresholds were chosen to make the likelihood of a false positive connection extremely small ${ }^{62,128}$, Fig. 2a-b and e, Supplementary Adjacency Matrix, Supplementary Atlas, Materials and Methods). We refer to the remaining pre-modulatory neurons as "other weakly connected partners". While they could also influence modulatory neuron activity, especially in combination with each other, we focus our study mainly on the reliably and numerically more strongly connected 102 pre-modulatory neuron pairs.

\section{Relationship between functional diversity and input diversity of MB modulatory neurons}

We wondered how the functional diversity of modulatory neurons relates to their input diversity. As expected, functionally distinct DANs (whose activation leads to aversive or appetitive memory for paired odors; Fig. 1c) that innervate distinct lobes receive inputs from distinct subsets of pre-modulatory neurons (Fig. 2e and Extended Data Fig. 3 ). We found that even some functionally distinct modulatory neurons that innervate the same compartment receive inputs from drastically different subsets of pre-modulatory neurons: for example DAN-g1 and OAN-g1 that express different neuromodulators and differ in their ability to induce memory (e.g. Fig. 1c, 2e and Extended Data Fig. 3 ). Such difference in input structure suggests that some modulatory neurons that innervate the same compartment may be differentially recruited during learning.

In contrast, functionally similar VL/LA-DANs (whose activation leads to aversive memory for paired odors) share a higher fraction of presynaptic partners with each other than with other DANs (Fig. 2e and Extended Data Fig. 3). Nevertheless, even these functionally similar VL/LA DANs receive input from distinct combinations of input neurons (Fig. 2e), potentially explaining why they have similar, but not identical tuning properties to different punishment types (Fig. 1f).

In summary, we found that each modulatory neuron type that is distinguishable based on the compartment it innervates, or based on neurotransmitter expression, receives input from a unique combination of neurons and thus potentially encodes a unique set of features. 
Feedback neurons reveal a highly recurrent architecture for computing teaching signals

We aimed to characterize the pre-modulatory neurons based on the inputs they receive. Specifically, we asked whether they can convey feedback information about previously formed memories (via input originating from MBONs), or about received US (via afferent input from sensory neurons), or both. Surprisingly, we found that the majority $(61 / 102)$ of pre-modulatory neurons receive feedback input from MBONs (Fig. 2a-c, Extended Data Fig. 4a-c). 40 left-right homologous neuron pairs receive reliable (as defined above) direct input from $\mathrm{MBONs}$, providing one-step feedback from MBONs to modulatory neurons (we call these one-step feedback neurons, FBNs, Fig. 2a-c, Extended Data Fig. 4a). Another 21 pre-modulatory neuron pairs receive reliable direct input from FBNs (but not MBONs) and provide two-step feedback from MBONs to modulatory neurons (we call these two-step feedback neurons, FB2Ns, Fig. 2a-c, Extended Data Fig. 4b). The majority of FBNs also receive input from other FBNs, providing two-step, as well as one-step feedback (Fig. 2b-c and Extended Data Fig. 4a). The remaining (41/102) premodulatory input neuron pairs do not receive reliable direct $\mathrm{MBON}, \mathrm{FBN}$, nor FB2N input, so we classified them tentatively as "feedforward neurons" (FFNs, Fig. 2a-c).

To determine the likelihood that MBONs could influence modulatory neuron activity, via the feedback pathways we also analyzed the fraction of total input that FBNs and FB2Ns receive from MBONs, and that modulatory neurons receive from feedback pathways. In previous studies we have demonstrated functional connections even when neurons received $2 \%$ of their input from another neuron ${ }^{62,124}$. We found that individual FBNs receive on average $12 \%$ of their total synaptic input from MBONs and $26 \%$ from MBONs and FBNs combined (Extended Data Fig. 4a and c). Similarly, individual FB2Ns receive on average $17 \%$ of their total synaptic input from FBNs and 28\% from FBNs and FB2Ns combined (Extended Data Fig. $4 \mathrm{~b}$ and $\mathrm{c}$ ). Based on these input fractions we expect that MBONs can significantly influence FBN and FB2N activity. Strikingly, we found that many modulatory neurons receive more than $50 \%$ of their total dendritic input from feedback pathways, including directly from MBONs, one-step, and two-step feedback (Fig. 2d). This suggests that modulatory neuron activity could be strongly modulated by MBON activity, via the newly discovered one-step and two-step feedback neurons.

\section{Multilevel convergence of afferent US pathways with feedback pathways}

We investigated how the feedback pathways from MBONs converge with afferent pathways from US sensory neurons. We focused on the VL/LA-DANs that we identified as responding to nociceptive and/or mechanosensory neuron activation (Fig. 1f) and asked whether they receive the somatosensory and $\mathrm{MBON}$ input via distinct or overlapping pre-modulatory neurons. In the larva, the early portions of the somatosensory circuits that process aversive cues are well characterized ${ }^{62,124,129,130}$. We had previously reconstructed all 1st order PNs downstream of nociceptive and mechanosensory sensory neurons, a subset of 2 nd order $\mathrm{PNs}$, and a few 3rd order $\mathrm{PNs}^{62,124}$.
This enabled us to search for shortest pathways from the nociceptive and mechanosensory sensory neurons to the VL/LA-modulatory neurons. We note that the pathways identified in this way represent only a subset of existing pathways, because not all of the $2 \mathrm{nd}$, 3rd and 4 th order somatosensory PNs have been reconstructed. Nevertheless, we were able to identify two-, three-, and four-step pathways from the nociceptive and mechanosensory sensory neurons to six different pre-modulatory neurons that target the VL/LA-modulatory neurons: three FFNs and three FB2Ns (Fig. 2f, Extended Data Fig. 5, Supplementary Adjacency Matrix, Supplementary Atlas). Thus, the afferent US pathways converge with feedback pathways from MBONs at multiple levels: both onto the modulatory neurons themselves (via FFNs) and onto the premodulatory FB2Ns.

\section{Modulatory neurons receive convergent one-step feedback from multiple MBONs from functionally distinct compartments}

Next, we analyzed in more detail the types of one-step feedback motifs formed by FBNs (Fig. 2a-c, 3a-c, Extended Data Fig. 6a-b, Supplementary Adjacency Matrix, and Supplementary Atlas). Specifically, we asked whether FBNs mostly provide input to their own compartment, or whether they link multiple compartments for forming distinct types of memories. We observed a surprising diversity of one-step FBNs that linked unique combinations of MBONs with unique combinations of modulatory neurons (Fig. 3a and Extended Data Fig. 6a-b). Some (7/40) FBNs provide exclusively withincompartment feedback (Fig. 3a). Some (13/40) provide exclusively cross-compartment feedback (we named individual neurons of this subset of FBNs, FANs, for feedacross neurons, Fig. 3a). Some (8/40) FBNs synapse onto multiple modulatory neurons from multiple compartments (Fig. 3a and Extended Data Fig. 6b). Interestingly, the largest class of FBNs (17/40, Fig. 3a, 3d, and Extended Data Fig. 6a) receives input from multiple MBONs and appears to be well suited for comparing odor drive to functionally distinct compartments of the MB (Fig. 1c). Thus, almost all of these FBNs (at least 13/17, and potentially more, but the neurotransmitters of all MBONs are not known) receive GABAergic (inhibitory) or glutamatergic (potentially also inhibitory ${ }^{131,132}$ in insects) input from MBONs from compartments implicated in memory formation, and cholinergic (excitatory) inputs from MBONs from other compartments (Fig. 3a, 3d, and Extended Data Fig. 6a). The comparison of inhibitory and excitatory input may enable these FBNs to more accurately read out the results of learning-induced plasticity ${ }^{55,85,133}$ in memory compartments, relative to other compartments.

We also found that most modulatory neurons receive input from multiple FBNs. For example, all ML-DANs and VL-DANs capable of evoking olfactory memory (Fig. 1c) received input from at least three different FBNs (Fig. 2e, 3a, and Extended Data Fig. 6b). Clustering FBNs based on the pattern of output to modulatory neurons revealed that some UVL, LVL and LA modulatory neurons stand out as prominent targets of feedback input, receiving significant input from clusters of 7 or more different FBNs (e.g. neurons forming aversive memory DAN-d1, DAN- 
a

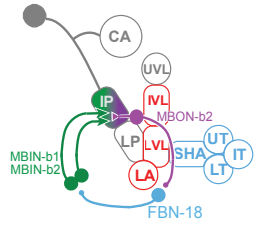

$\underset{\rightarrow \text { Type of connection }}{\rightarrow \text { excitatory (ChAT) }}$ $\rightarrow$ excitatory (ChAT)
$\rightarrow$ inhibitory (GABA)

$\rightarrow$ probably inhibitory (GluT) $\rightarrow$ unknown
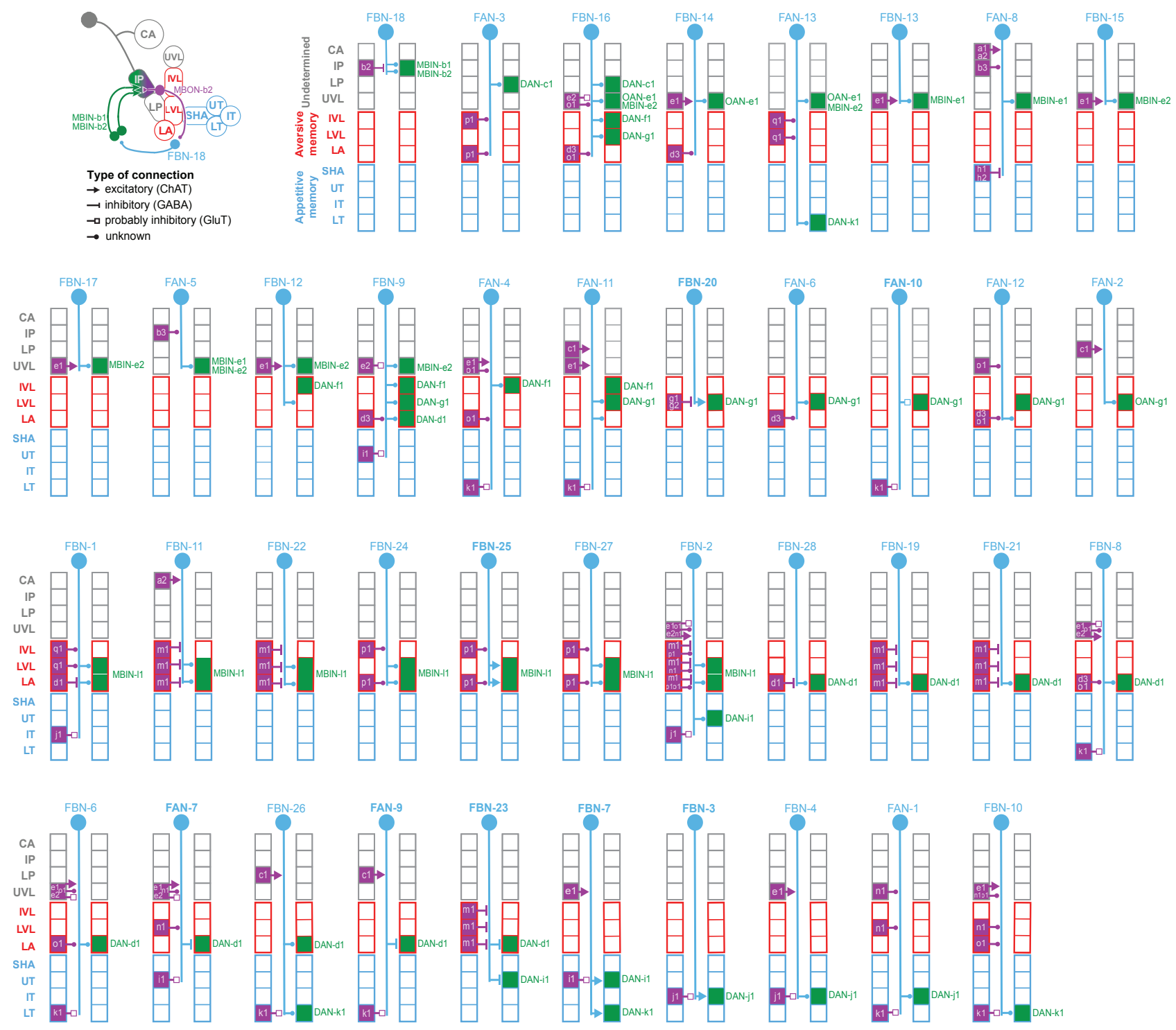

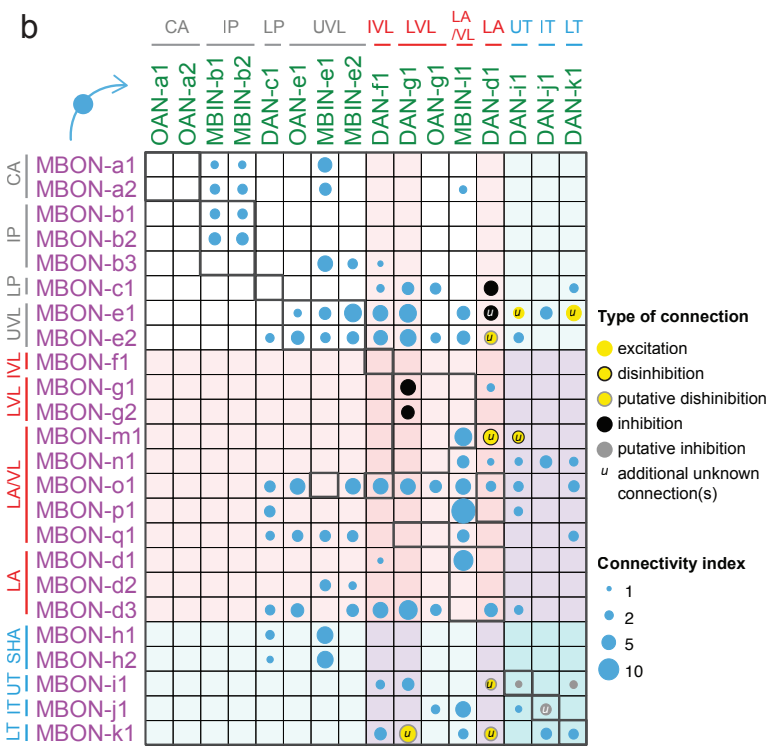

Figure 3 || Modulatory neurons receive convergent one-step feedback from multiple MBONs from functionally distinct compartments

a Connectivity of each of the 40 feedback neuron (FBN) pairs that provide one-step feedback from MBONs to DANs. Each diagram represents the connectivity of a single left-right pair of homologous FBNs. Each box indicates a separate compartment. Purple, compartment(s) of the presynaptic $\operatorname{MBON}(\mathrm{s})$. Green, compartment(s) of the postsynaptic modulatory neuron(s). d

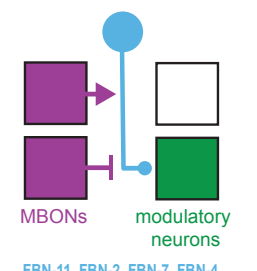

FBN-11, FBN-2, FBN-7, FBN-4,

FAN-9, FAN-8, FAN-4, FBN-10)

e

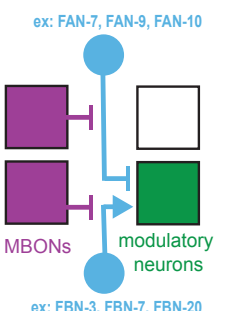

C

FBNs are ordered according to the modulatory neuron they innervate, starting with peduncle modulatory neurons and ending with the medial lobe ones. Classical neurotransmitter profiles of the MBONs and FBNs are indicated by the arrow (cholinergic, excitatory connection), vertical line (GABAergic, inhibitory connection) or square (glutamatergic, probably also inhibitory connection) for the neurons for which they are known from immunostaining (For images, see Extended Data Fig. 10 for FBNs and Eichler et al. 2017 (Continues into the next page.) 
(Continues from prior page, Figure 3.)

Extended Data Fig. $2 b$ for MBONs), or by a circle when they are unknown. 7 FBNs provide exclusively within-compartment feedback. 13 FBNs provide exclusively cross-compartment feedback (named FANs, for feed-across). 8 FBNs synapse onto multiple modulatory neurons from multiple compartments. The largest class of FBNs (17) receives input from multiple MBONs, with the majority (at least 13 and maybe more) receiving input of potentially opposite sign from MBONs from functionally distinct compartments.

b Modulatory neurons receive input from multiple MBONs from functionally distinct compartments via the FBNs. Connectivity matrix shows connections between MBONs and modulatory neurons via the indirect one-step feedback pathways obtained by multiplying the $\mathrm{MBON} \rightarrow \mathrm{FBN}$, and $\mathrm{FBN} \rightarrow$ modulatory neuron connectivity matrices (normalized as in Fig. 2b and including all connections where the presynaptic neuron accounts for at least $1 \%$ of input onto the postsynaptic neuron). A connectivity index was computed by taking the square root of the numbers in the resulting matrix product. A connectivity index of 1, 10, and 100 means that for both connections comprising that indirect feedback pathway the presynaptic neuron accounts for $1 \%, 10 \%$, and $100 \%$ of input onto that postsynaptic neuron, respectively. When the neurotransmitters of both the MBON and the FBN(s) that comprise a connection are known, the circle is color-coded to represent types of connection: excitatory (ChAT and ChAT), disinhibitory (GABA and GABA), probably disinhibitory (GluT and GABA/GluT), inhibitory (GABA and ChAT), probably inhibitory (GluT and ChAT). Color shades represent the valence of the memory formed in a given compartment (red: aversive memory, blue: appetitive memory). True within-compartment feedback connections from an MBON that receives direct synaptic input from that modulatory neuron are boxed in bold (e.g. from MBON-g1 and MBON-g2 onto DAN-g1). Some multicompartment MBONs provide feedback to modulatory neurons from the same compartment that do not synapse onto them directly, and we do not consider these to be true within-compartment feedback connections (e.g from MBON-m1 onto DAN-d1). Note that all four true within-compartment feedback connections with known neurotransmitters are potentially inhibitory: MBON-g1 to DAN-g1, MBONg2 to DAN g2, MBON-i1 to DAN-i1 and MBON-j1 to DAN-j1. In

g1, and neurons of unknown function MBIN-11, MBINe1 and -e2, Extended Data Fig. 6b and 7). Similarly, clustering FBNs based on the input from MBONs revealed that specific VL-MBONs stood out as prominent sources that provide output to clusters of FBNs (for example, the cholinergic UVL MBON-e1 strongly targets a cluster of 13 different FBNs, Extended Data Fig. 6a and 8).

Since most FBNs receive input from multiple MBONs, and most modulatory neurons receive input from multiple FBNs, we analyzed the connections from all MBONs to all modulatory neurons via all possible one-step feedback pathways (by multiplying normalized MBON-to-FBN and FBN-to-modulatory neuron connectivity matrices from Supplementary Adjacency Matrix). We found that most modulatory neurons received indirect one-step feedback from many MBONs (Fig. 3b). Most compartments therefore received one-step feedback from many other compartments (Fig. 3c). All modulatory neurons except those that innervate CA and IP received one-step feedback from each of the three functionally distinct regions of the MB: UVL (unknown function), VL aversive memory compartments, and ML appetitive memory compartments (Fig. $3 \mathrm{~b}-\mathrm{c})$. This is in stark contrast to the direct connectivity from MBONs to modulatory neurons, which is very sparse and connects very few compartments (Extended data fig. 9a-b). Thus, the newly discovered FBNs greatly increase the connectivity between MBONs and modulatory neurons, enabling the output from multiple functionally distinct regions of the MB to influence the activity of a single modulatory neuron during memory formation. contrast, many cross-compartment connections are potentially excitatory or disinhibitory.

c All compartments except CA and IP receive one-step feedback from multiple compartments and from each of the three functionally distinct regions of the MB. Matrix shows the relative one-step connection strength index from a row compartment to a column compartment via FBNs. Strength is calculated as in $\mathbf{b}$, but pooled (summed and normalized) for all MBONs from a compartment and all modulatory neurons from a compartment, and then multiplied by 100 . Note the compartments of the VL (vertical lobe: UVL, IVL, LVL) and LA (lateral appendix) are strongly interconnected. The color shades are as in $\mathbf{b}$.

d-e Summary diagram of commonly observed convergence motifs. Feedback connections of opposite sign from functionally distinct compartments converge onto FBNs and DANs. In each diagram, the FBN (blue) receives direct input from one or more $\mathrm{MBON}(\mathrm{s})$ (purple), and connects with the postsynaptic modulatory neuron(s) (green). Each box denotes a separate compartment. The type of connection (GABAergic, glutamatergic, cholinergic, or unknown) is represented by different arrowheads as described in the legend in a. d More than a quarter of FBNs (at least 12, and potentially more) receive direct GABAergic (inhibitory) or glutamatergic (also potentially inhibitory) input from MBONs from one compartment and direct cholinergic (excitatory) input from MBONs from a functionally distinct compartments enabling them to compare the odor drive to these MBONs. e Many DANs (DAN-f1, d1, i1, j1, and k1) receive potentially inhibitory (excitatory $\mathrm{FBN}$ downstream of an inhibitory MBON) one-step feedback from MBONs from one compartment and potentially disinhibitory (inhibitory FBN downstream of an inhibitory MBON) or excitatory (excitatory FBN downstream of an excitatory MBON) one-step feedback from MBONs from a functionally distinct compartment. A common pattern for the lobe DANs implicated in memory formation may be a likely inhibitory connection from an MBON from their own compartment and a likely disinhibitory connection from an MBON from a compartment of opposite valence (observed for both DAN-g1 and i1), that could enable these DANs to compare the odor drive to MBONs from compartments of opposite valence.

\section{A modulatory neuron receives inhibitory and ex- citatory feedback from compartments of opposite valence}

To gain a better understanding of how feedback motifs could influence modulatory neuron activity we wanted to determine which feedback neurons were excitatory and which inhibitory. We were able to identify GAL4 lines ${ }^{134}$ that drive expression in eight different FBNs and three FB2Ns. We could therefore label these feedback neurons with $\mathrm{GFP}^{135}$ and test whether they express GABA $^{136}$, vesicular glutamate transporter (vGlut) ${ }^{137}$, or choline acetyl transferase (ChAT) ${ }^{138}$ using immunohistochemistry. Unfortunately, while these GAL4 lines were selective enough to allow visualization of the relevant neurons, most lines were not selective enough to enable targeted manipulation for functional connectivity and behavioral analysis. We found that four of the tested FBNs were cholinergic (i.e. excitatory), three were GABAergic (i.e. inhibitory), and one was glutamatergic (possibly also inhibitory ${ }^{131,132}$, Fig. 3a and Extended Data Fig. 10). Two FB2Ns were glutamatergic and one was cholinergic (Extended Data Fig. 10).

For a few cases where we could identify the neurotransmitter profiles of both the $\mathrm{MBON}^{47}$ and the $\mathrm{FBN}$ in a one-step feedback connection, we attempted to predict the signs of these connections (Fig. 3b). All of the true withincompartment feedback connections with known neurotransmitters were potentially inhibitory (4/4), comprising a GABAergic or glutamatergic MBON and an excitatory 
Figure 4 || Functional inhibitory and excitatory feedback connections from compartments of opposite valence converge onto a DAN.

a Schematic diagram showing indirect inhibitory withincompartment feedback and disinhibitory feedback from a compartment of opposite valence converging onto the same DAN, as predicted based on connectivity and neurotransmitter profiles. These two types of predicted connections are tested separately using optogenetic activation of the MBONs and patch-clamp recording of a DAN (b-d and e-g). Boxes denote compartments. Blue and red outlines, appetitive and aversive memory compartments, respectively. Purple, light blue, and green, MBONs, FBNs, and DANs, respectively.

b The cholinergic FBN-7 downstream of the glutamatergic medial lobe MBON-i1 could mediate inhibitory one-step withincompartment feedback onto DAN-i1.

c Whole-cell patch-clamp recording of DAN-i1 (medial lobe) during optogenetic activation of the medial lobe MBON-i1 of the same compartment reveals inhibitory feedback at the onset or offset of MBON-i1 activation. The action potentials of all 180 electrophysiologically recorded traces from 9 animals in response to MBON-i1 activation (purple bar) are shown as raster plots. Dashes on the left separate rasters belonging to distinct animals. We observed two types of inhibitory responses in DAN-i1 to MBON-i1 activation: a long-latency inhibitory response to the onset of MBON-i1 activation in $3 / 9$ animals (shown at the top, $55.3 \pm 17.3 \mathrm{~ms}, \mathrm{n}=60$ traces from 3 animals), and an even longer latency inhibitory response to the offset of MBON-i1 activation in 4/9 animals (shown in the middle, $95.3 \pm 43.5 \mathrm{~ms}, \mathrm{n}=80$ trials from 4 animals).

In two animals (shown at the bottom) DAN-i1 showed no response to MBON-i1 activation ( $\mathrm{n}=40$ trials). Inhibition at the onset in some animals but not others might result from distinct baseline states of FBN, as shown in the model in Extended Data Fig. 11b. Inhibition at the offset might be result of post-inhibitory rebound within a two-step feedback pathway as shown in Extended Data Fig. 11c. d An example individual trace from c. More examples are shown in Extended Data Fig. 11a.

e The GABAergic FBN-23 downstream of the GABAergic vertical lobe MBON-m1 could mediate a disinhibitory one-step connection

FBN (Fig. 3b and 3e, MBON-g1 to DAN-g1, MBON-g2 to DAN-g1, MBON-i1 to DAN-i1 and MBON-j1 to DAN-j1). In contrast, most of the $(8 / 11)$ cross-compartment connections with known neurotransmitters were potentially functionally excitatory, either disinhibitory (comprising an inhibitory MBON and an inhibitory FBN), or excitatory (comprising an excitatory MBON and an excitatory FBN, Fig. 3b and 3e). Out of those, all of the connections between compartments of opposite valence (4/4) were potentially disinhibitory (Fig. 3b and 3e). Furthermore, we observed that some modulatory neurons (e.g. DAN-g1 and DAN-i1) received both potentially inhibitory feedback from their own compartment and potentially excitatory feedback from compartments of opposite valence (Fig. 3b and $3 \mathrm{e}$ ).

We designed experiments to functionally confirm the two types of predicted feedback connections onto the same DAN (Fig. 3e, 4a-g). We were able to identify a strong LexA line for DAN-i1 ${ }^{78}$. DAN-i1 receives potentially inhibitory one-step feedback from the glutamatergic MBONi1 in its own compartment (via the excitatory FBN-7, Fig. 4a-b), and potentially disinhibitory one-step feedback from the GABAergic MBON-m1 from compartments of opposite valence (via the GABAergic FBN-23, Fig. 4a and 4e). Neither of these MBONs synapses directly onto DAN-i1. We also generated Split-GAL4 lines to selectively express Chrimson in MBON-i1 or in MBON-m1. We activated these MBONs optogenetically while recording intracellularly from DAN-i1 (labelled with GFP using the a

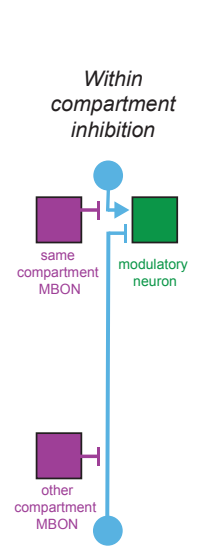

b Possible pathway for inhibition at the onset:

C MBON-i1 > CsChrimson MBON-i1

d

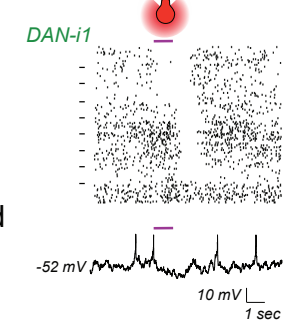

e Possible pathway for disinhibition:

f

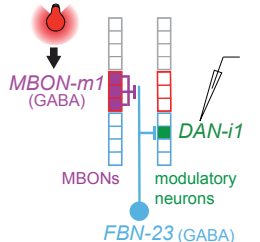

onto the medial lobe DAN-i1. The vertical lobe MBON-m1 receives input from DAN-g1, whose activation can induce aversive memory. In contrast, the medial lobe DAN-i1 activation induces appetitive memory.

f Whole-cell patch-clamp recording of DAN-i1 during optogenetic activation of the medial lobe MBON-m1 from a compartment of opposite valence reveals an excitatory cross-compartment feedback connection. The action potentials of all the 45 electrophysiologically recorded traces from the 3 animals in response to $\mathrm{MBON}-\mathrm{m} 1$ activation (purple bar) are shown as raster plots. In response to optogenetic activation of MBON-m1, we observed a long-latency excitatory response in DAN-i1 in $3 / 3$ animals tested $(51.3 \pm 7.7 \mathrm{~ms}$, $\mathrm{n}=45$ trials from 3 animals).

g An example individual trace from $\mathbf{f}$ (more examples in Extended Data Fig. 11d).

\section{LexA line).}

Activating the glutamatergic MBON-i1 evoked longlatency (55ms +/-17) inhibitory responses in DAN-i1 on 9/27 trials (on all 3 trials in 3/9 animals, Fig. 4c-d, Extended Data Fig. 11a). The inter-animal variability could potentially be due to different baseline activity levels of FBNs mediating this connection: activating an inhibitory MBON can only lead to detectable inhibition of the DAN if the excitatory FBN targeted by the MBON has baseline activity, as illustrated by the simple rate-model in Extended data Fig. 11b. On 12/27 trials (on all 3 trials in 4/9 animals, Fig. 4c and Extended Data Fig. 11a) we observed very long latency inhibitory responses to the offset of MBON-i1 activation only. These inhibitory responses to the offset had a longer latency $(95 \mathrm{~ms}+/-44)$ than the inhibitory responses to the onset $(55 \mathrm{~ms}+/-17)$ of MBON-i1 activation and could therefore be mediated by a longer two-step feedback pathway (as proposed in the Extended Data Fig. 11c).

In contrast, we found that activating the GABAergic MBON-m1 evoked excitatory responses in DAN-i1 on $9 / 9$ trials (on all 3 trials in $3 / 3$ animals) with a similar latency $(47 \mathrm{~ms}+/-9)$ to the inhibitory responses evoked by MBON-i1 activation (Fig. 4e-g and Extended Data Fig. 11d). In summary, we confirmed with physiological recording an inference we had made from structural connectivity and neurotransmitter information: that functionally inhibitory and excitatory MBON connections from compartments of opposite valence converge onto the 
a

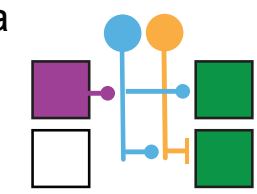

example: $F B 2 N-18$

$\rightarrow$ excitatory (ChAT) $\rightarrow$ inhibitory (GABA)

$\rightarrow$ probably inhibitory (GluT)

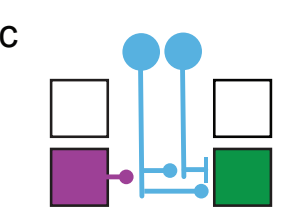

example: $F B N-23$

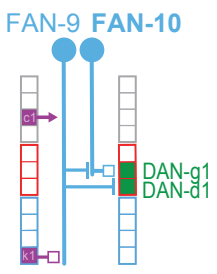

b
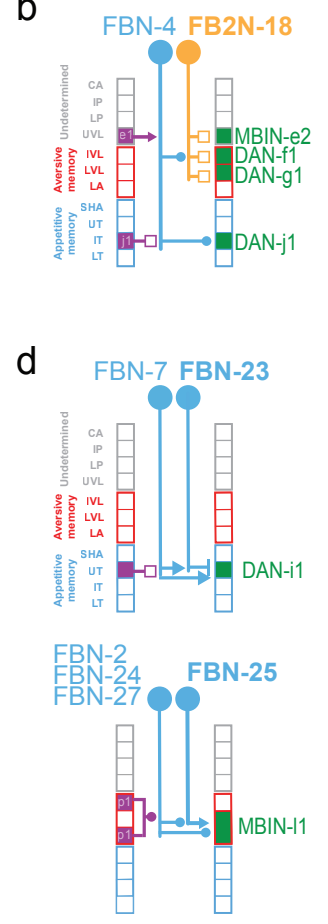
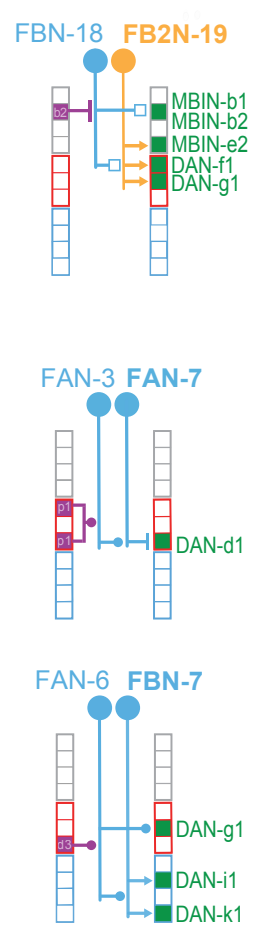

e
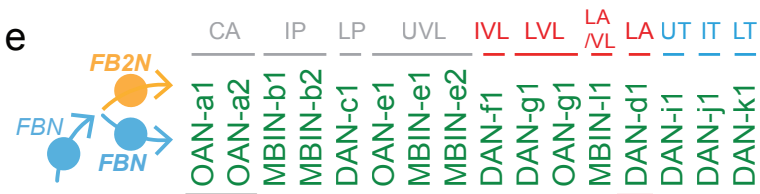

ऽ)

MBON-a1

MBON-a2

MBON-b1

MBON-b2

MBON-b3

I MBON-c1

MBON-e1

MBON-e2

$\sum \mathrm{MBON}-\mathrm{f1}$

\pm MBON-g1

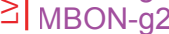

MBON-m1

MBON-n1

MBON-01

MBON-p1

MBON-q1

MBON-d1

$\unlhd$ MBON-d2

MBON-d3

II MBON-h1

का $\mathrm{MBON}-\mathrm{h} 2$

5I MBON-i1

니 MBON-j1

IMBON-k1
Figure 5 || Two-step feedback from most MBONs to most modulatory neurons further increases inter-compartment connectivity.

a Schematic diagram of a two-step feedback motif involving an FBN (blue) and an inhibitory FB2N (yellow). The FBN provides one-step feedback to some compartments and two-step feedback to others via the FB2N.

b Two example two-step within-compartment feedback motifs involving FB2Ns with identified neurotransmitters. In the first example, FBN-4 integrates opposite drives from MBON-e1 and MBON-j1 and transmits its signal directly to ML DAN-j1 and indirectly to VL modulatory neurons via the glutamatergic FB2N-18. In the second example, FBN-18 distributes a disinhibitory signal from MBON-b2 to many modulatory neurons both via one-step feedback and twostep feedback involving the cholinergic FB2N-19. Arrowheads denote the type of synaptic connection as in $\mathbf{a}$.

c Schematic diagram of a two-step feedback motif involving two FBNs (blue) rather than an FBN and an FB2N. The FBN provides one-step feedback to some compartments and two-step feedback to others via another FBN.

same DAN (Fig. 4a-g). DANs that receive this pattern of feedback could compare the odor-evoked excitation of MBONs in compartments of opposite valence and thereby compute the integrated predicted value of an odor across aversive and appetitive memory systems.

\section{Two-step feedback from most MBONs to most modulatory neurons further increases inter- compartment connectivity}

Next, we investigated in more detail the two-step feedback motifs (Fig. 2a-c, 5a-e, Extended Data Extended Data Fig. 12, 13a-d, and 14, Supplementary Adjacency Matrix and Supplementary Atlas). We found 21 FB2N pairs, which do not themselves receive direct MBON input, but do receive input from FBNs, thus providing twostep feedback from MBONs to modulatory neurons (Fig. 2a-c, 5a-b, Extended Data Fig. 4b-c, Extended Data Fig 12 and 13b-c, Supplementary Adjacency Matrix and Sup- d Five example two-step within-compartment feedback motifs involving FBNs with identified neurotransmitters. In these examples, FBN-23, FAN-7 or FBN-25 provide within-compartment two-step feedbacks onto DAN-i1, DAN-d1 and MBIN-l1 respectively. FAN10 and FBN-7 distribute the signal of their presynaptic FBN to modulatory neurons in other compartments. The type of synaptic connection is symbolized by different arrowheads as in a.

e Most modulatory neurons receive two-step feedback from most MBONs via the FBNs and FB2Ns. Connectivity matrix shows connections between MBONs and modulatory neurons via twostep feedback pathways, obtained by multiplying the $\mathrm{MBON} \rightarrow \mathrm{FBN}$, $\mathrm{FBN} \rightarrow \mathrm{FB} 2 \mathrm{~N} / \mathrm{FBN}$ and $\mathrm{FB} 2 \mathrm{~N} / \mathrm{FBN} \rightarrow$ modulatory neuron connectivity matrices (normalized as in Fig. $\mathbf{2 b}$ and including all connections where the presynaptic neuron accounts for at least $1 \%$ of input onto the postsynaptic neuron). The connectivity index was computed by taking the cubic root of the numbers in the resulting matrix product. A connectivity index of 1, 10, and 100 means that for the three connections comprising that indirect feedback pathway the presynaptic neuron accounts for 1\%,10\%, and $100 \%$ of input onto that postsynaptic neuron, respectively.

plementary Atlas). Many FBNs also receive input from other FBNs (Fig. 5c-d, Extended Data Fig. 4a, 4c, 13a and 14) and provide both two-step and one-step feedback. We therefore also analyzed the connections between all MBONs to all modulatory neurons via the two-step pathways (by multiplying the MBON-FBN, FBN-FB2N/FBN and FB2N/FBN-modulatory neuron normalized connectivity matrices from Supplementary Adjacency Matrix). We found two-step feedback from most MBONs to most modulatory neurons further increases inter-compartment connectivity (Fig. 5e and Extended Data Fig. 9b). We were able to determine neurotransmitter profiles for seven neurons that provide two-step feedback: three (FBN-25, FBN-7, and FB2N-19) were cholinergic, two (FBN-23 and FAN-7) were GABAergic, and two (FAN-10 and FB2N18) were glutamatergic (Fig. 5b and d, Extended Data Fig. 10). In summary we found a diverse set of twostep feedback motifs that could support within- and crosscompartment computations. 
EsobibBxily prepxintldioi: thttps:/2do1. @rg/10.1101/649731; this version posted May 27, 2019. The copyright holder for this preprint (which was not certified by peer review) is the author/funder. All rights reserved. No reuse allowed without permission.

a

FB2N-19

Cholinergic
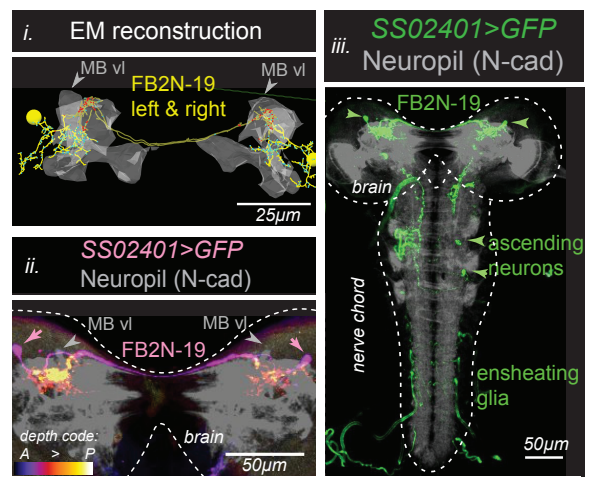

b

FAN-7

GABAergic
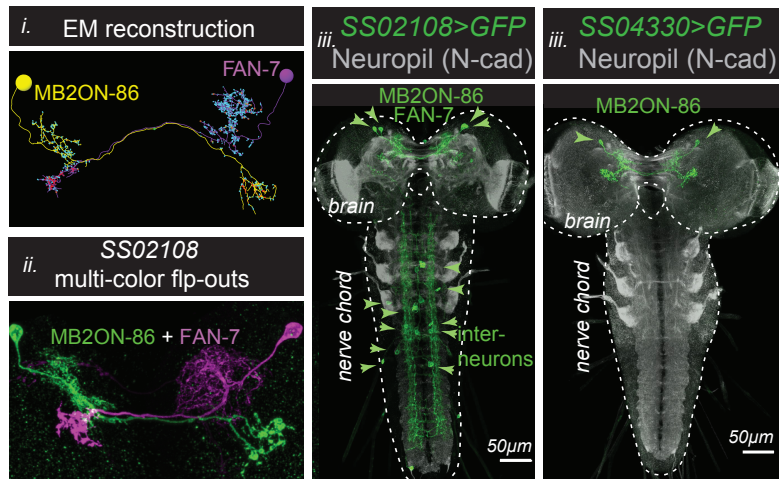

C

FB2N-18 / FB2N-11

Glutamatergic
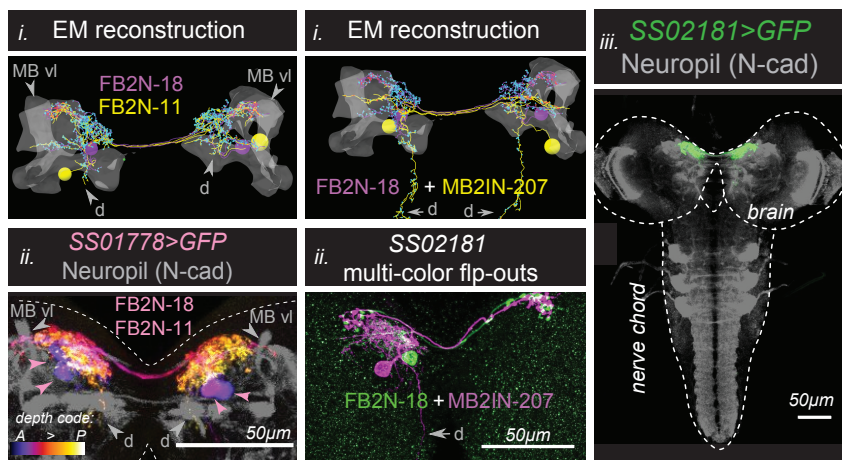

Figure 6 || Feedback neurons can drive associative memory formation.

We were able to generate Split-GAL4 lines that drive expression in a single pair of neurons, or in very few cell types, for three different pairs of FBNs or FB2Ns that target VL DANs (a-c). We used these lines to optogenetically activate these neurons instead of a US during an associative learning paradigm (d-f).

a-c Identification of driver lines for EM-reconstructed neurons. i) Skeletons of specific feedback neurons reconstructed in the EM. Red dots, presynaptic sites. Blue dots, postsynaptic sites. Grey, mushroom body vertical lobe $(M B v l)$ for reference. $d$, dendritic arbor. ii) Maximum intensity projections of confocal stacks of larval brains showing the same neurons visualized with reporters targeted using specific Split-GAL4 lines. For some lines multicolor FLP-outs were used to visualize each neuron in a different color to facilitate identification and comparison with EM. Grey, neuropil visualized with Ncad. Dashed line, brain outline. iii) Maximum intensity projections of confocal stacks of the entire nervous system showing the complete expression pattern of each line revealed by driving UAS-myr-GFP. Grey, neuropil visualized with N-cad. Dashed line, nervous system outline.

a, The SS02401-Split-GAL4 line drives expression in FB2N-19 $(i)$ in the brain $(i i)$, and very weakly and stochastically (not reproducibly in all samples) in a few ascending neurons and ensheathing glia in the d
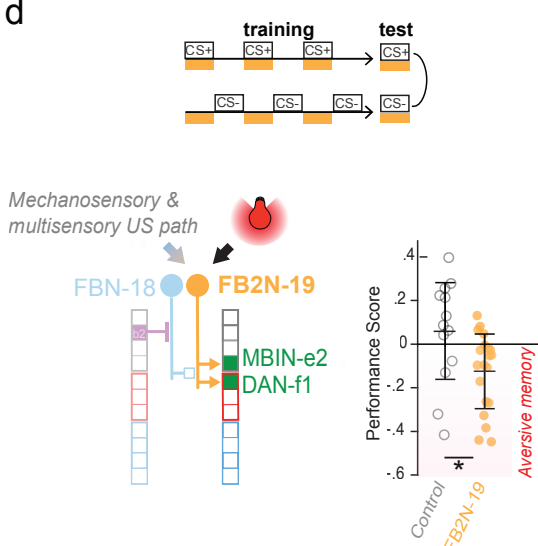

e
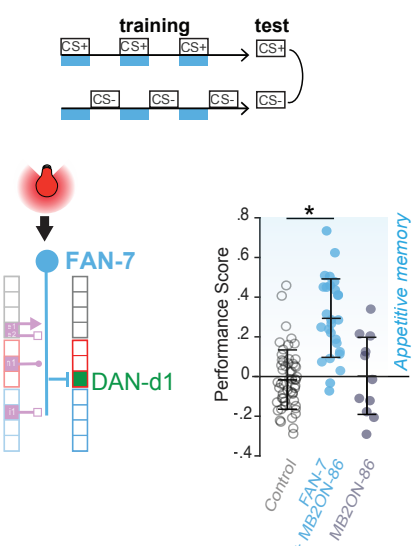

$\mathrm{f}$
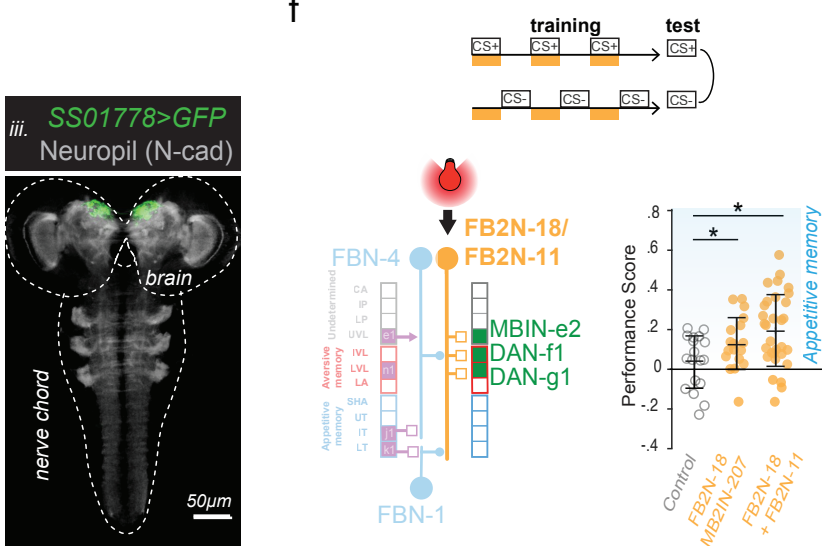

nerve chord that are unlikely to have an ability to evoke associative memory, due to weak and stochastic expression $(i i i)$.

b, The SS02108-Split-GAL4 line drives expression in FAN-7 and MB2ON-86 $(i)$ in the brain visualized with multicolor flp-outs in $(i i)$. Complete expression pattern of SS02108-Split-GAL4 visualized with $U A S$-myr-GFP shows additional expression in a few somatosensory interneurons in the nerve cord, called ladders, that mediate avoidance behavior and are hence unlikely to have a positive valence and evoke an appetitive memory. We identified the SS04330-Split-GAL4 line as driving expression specifically in the MB2ON-86 neuron and used it as an additional control in e.

c, The SS01778-Split-GAL4 line drives expression in both FB2N-18 and FB2N-11, which have very similar morphology and very similar connectivity (Extended Data Fig. 12 and 13b-d). They both connect strongly to DAN-f1 and weakly (but reliably) to MBIN-e2; FB2N18 also connects weakly (but reliably) to DAN-g1 (Extended Data Fig. 13c). The SS02181-Split-GAL4 line ( $i i$ shows multi-color flpouts) drives expression in FB2N-18 and in MB2IN-207, one of the weakly connected pre-modulatory neurons from lineage DAMv12. Notice the ventrally projecting dendrite $(d)$, a distinctive feature of MB2IN-207 neuron $(i)$. UAS-myr-GFP expression patterns of the two lines show that they do not drive expression in any other neurons in the nerve cord $($ iii).

(Continues into the next page.) 
(Continues from prior page, Figure 6.)

d-f Pairing the optogenetic activation of the neurons in these lines with the odor ethyl acetate as a CS (as in Fig. 1b-c) induces associative memory. Plotted are the learning performance scores (computed as described in Fig. 1b) obtained after paired or unpaired optogenetic activation of these neurons with odor, compared to a corresponding empty line w;attp40;attP2>UAS-CsChrimson (open circle) as a control. Horizontal lines indicate means and standard deviations of the individual data points; ${ }^{*} \mathrm{P}<0.05$ Mann-Whitney U-test comparison between groups.

d, Optogenetic activation of the excitatory cholinergic FB2N-19 that is presynaptic to DAN-f1 and MBIN-e2 (with SS02401-Split-GAL4 line) induces aversive memory (yellow), same as the activation of its presynaptic DAN-f1 (Fig. 1c). Note that FB2N-19 has to be activated during the test for memory expression as is the case for many punishing stimuli for Drosophila larva (Gerber et al., 2009).

\section{Feedback neurons can drive memory formation}

So far, we have shown that at least some of the indirect feedback connections from MBONs to DANs are functional (Fig. 4b-g). However, we also wanted to test whether the feedback neurons can sufficiently influence DAN activity to actually induce learning. We succeeded in generating Split-GAL4 lines ${ }^{102}$ that drive expression in one or very few neuron types for: a cholinergic FB2N, a glutamatergic FB2N, and a GABAergic FBN that project onto DANs whose activation can induce aversive memory (Fig. 6a-c, Extended Data Fig. 1). We asked whether optogenetic activation of these feedback neurons (without directly activating any modulatory neurons) was sufficient to induce memory in our olfactory training paradigm (Fig. $6 \mathrm{~d}-\mathrm{f})$. We found that pairing of an odor with activation of the excitatory cholinergic FB2N-19 induces aversive memory (Fig. 6d and Extended Data Fig. 2a-b), similar to direct activation of its postsynaptic DAN-f1 (Fig. 1c). In contrast, pairing of an odor with the activation of the GABAergic FAN-7 induces appetitive memory (Fig. 6e and Extended Data Fig. 2a-b), opposite to direct activation of its post-synaptic DAN-d1 (Fig. 1c). The line that drives expression in FAN-7 also drives expression in another neuron pair in the brain (MB2ON-86, Fig. 6c), but pairing an odor with the activation of that neuron alone did not induce memory (Fig. 6e and Extended Data Fig. $2 \mathrm{a}-\mathrm{b})$. The line also drives expression in a few somatosensory interneurons in the nerve cord, but somatosensory pathways are expected to induce aversive memory (Fig. 1e). Similarly, pairing of an odor with the activation of the glutamatergic FB2N-18 and FB2N-11 (likely also inhibitory) induces appetitive memory (Fig. 6f, and Extended Data Fig. 2a-b), opposite to direct activation of their post-synaptic DAN-f1 and DAN-g1 (Fig. 1c). Thus, at least some feedback neurons can induce memory formation. Interestingly, activation of inhibitory feedback neurons induces memories of opposite valence to the activation of the DANs that they inhibit.

Connectivity-constrained model of the circuit reveals feedback neurons improve performance on complex learning tasks

To explore the computational consequences of the feedback neurons, we developed a model of the circuit constrained by i) the connectome, ii) the known neurotransmitter identities of $\mathrm{MBONs}^{47}$ and pre-modulatory neurons (Extended Data Fig. 10), and iii) the valences of compartments whose modulatory neuron activation evokes aver- e, Optogenetic activation of the inhibitory GABAergic FAN-7 ( SS02108-Split-GAL4 line) induces appetitive memory (dark blue), opposite to the aversive memory induced by the activation of its presynaptic DAN-d1(Fig. 1c). SS02108-Split-GAL4 line drives expression both in FAN-7, as well as in MB2ON-86, but pairing the activation of MB2ON-86 alone with odor (to disambiguate) (using the SS04330-Split-GAL4 line) did not evoke any memory (dark grey). f, Optogenetic activation of the glutamatergic FB2N-18 and FB2N11 together (with the SS01778-Split-GAL4 line) induces appetitive memory (yellow), opposite to activation of their presynaptic DANs-f1 and -g1 (Fig. 1c). Even activation of the glutamatergic FB2N-18 without FB2N-11, but with another weakly connected pre-modulatory neuron which is unlikely to be able to significantly influence modulatory neuron activity (with the SS02181-Split-GAL4 line) induces appetitive memory.

sive or appetitive memory when paired with odor (Fig. 1c). We modeled modifications of $\mathrm{KC}$ to MBON connections using a synaptic plasticity rule that depends on the timing of $\mathrm{KC}$ and modulatory neuron activity, consistent with findings in larval ${ }^{58}$ and adult Drosophila ${ }^{84,133,139}$. We optimized the model using gradient descent to perform various associative learning tasks ${ }^{140}$ (see Materials and Methods) and assessed the contributions of different feedback types by repeating the optimization procedure for networks lacking such feedback and comparing their performance. Tasks included first-order conditioning, secondorder conditioning, extinction, and context-dependent conditioning (Fig. 7a-b). First-order conditioning and extinction have been demonstrated in adult ${ }^{5,13,14,53}$ and larval Drosophila ${ }^{141}$. While second-order ${ }^{7,20}$ and contextdependent $^{22,142,143}$ conditioning have so far been investigated only in adult insects, we used these as example tasks to probe the ability of the circuit to support conditioning paradigms requiring additional computations. In the case of second-order conditioning, a reinforcement predicting conditioned stimulus is used to reinforce a second stimulus, while in context-dependent conditioning, the US valence depends on a previous contextual input.

We found that the performance on all tasks was significantly degraded in the absence of all feedback, including direct MBON feedback, one-step feedback via FBNs, and two-step feedback via FB2Ns and FBNs (Fig. 7a-b). The removal of the indirect feedback alone (with intact direct MBON feedback) also significantly degraded the performance on all tasks, with especially strong effects on the more complex tasks (Fig. 7a-b). Even the removal of twostep feedback alone significantly diminished performance on two of the more complex tasks (second-order conditioning and context-dependent conditioning), with a drastic effect on context-dependent conditioning (Fig. 7a-b). Thus, each additional feedback layer improves the performance of the network when it is tested on challenging associative learning tasks.

We also constructed networks lacking one- and twostep feedback only within or only across compartments. Removal of within-compartment feedback diminished performance on all tasks, while removal of cross-compartment communication substantially reduced performance for second-order conditioning (Fig. 7a-b). In total, each of the feedback pathways identified by the reconstruction may be important for associative learning paradigms that require computations such as prediction, prediction error, or context dependence. 
a

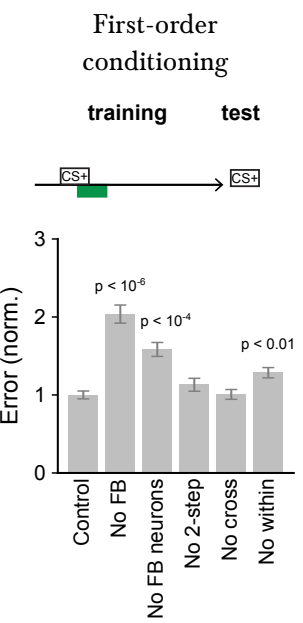

C

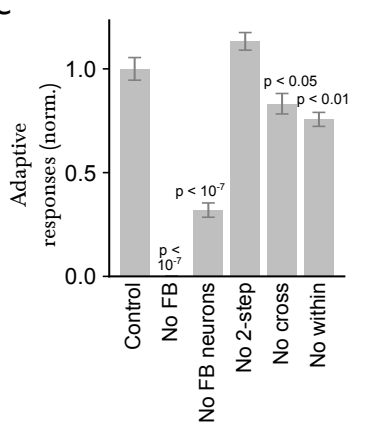

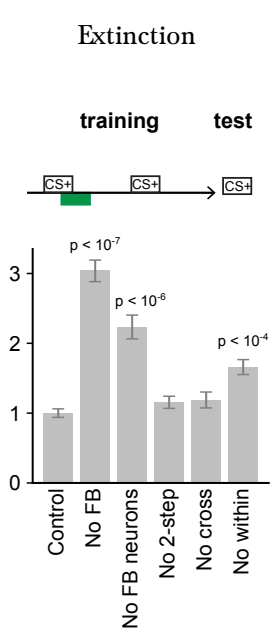

d

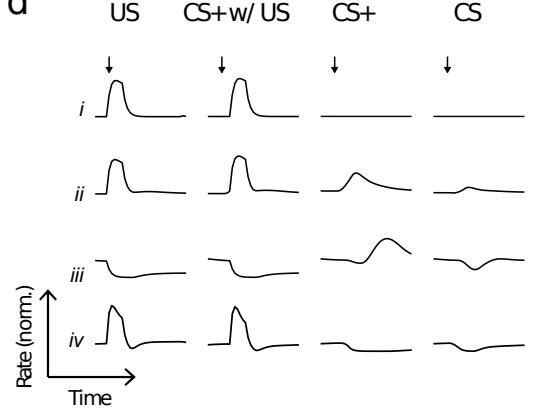

b conditioning
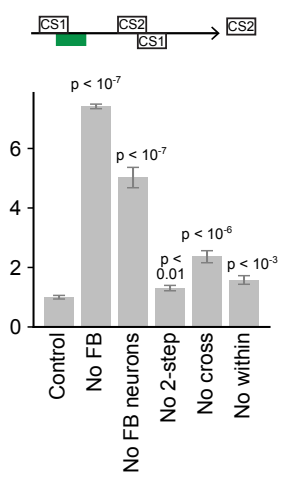

Context-dependent conditioning
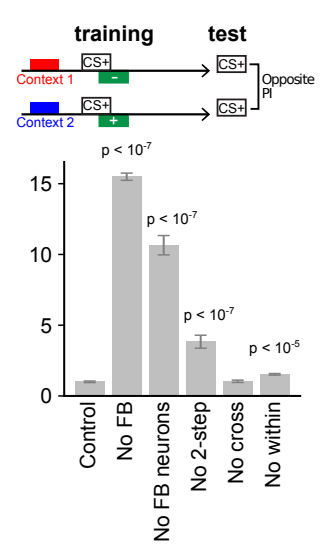

Figure 7 || Model of the circuit reveals how each type of feedback connection improves performance on distinct learning tasks.

a Normalized error (meansquared difference between actual and and target PI, normalized to the error for control networks) after optimizing models to perform first-order conditioning, extinction, and second-order conditioning. Error is shown for six cases: Control: full network, No FB: networks in which all feedback onto modulatory neurons, including direct MBON connections, is removed, No FB neurons: networks in which indirect FBN/FB2N feedback is removed but direct MBON connections are intact, No 2-step: networks in which only FB2Ns and all FBN-to-FBN connections are removed, No cross: networks in which indirect cross-compartment connections are removed, No within: networks in which indirect within-compartment connections are removed.

b Similar to a, but for networks optimized only to perform a context-dependent conditioning task.

c Adaptive response index for the networks in a, defined as the magnitude of the change in firing rate responses to CS+ presentation before and after conditioning, averaged over modulatory neurons. The results are normalized by the value for control networks. d Selected example responses of DANs from networks in a to US alone (US), CS+ paired with US following training (CS+ w/ US), to CS+ alone after training (CS+), and to CS prior to training (CS). Row $i$ : A DAN selective only to US that does not show adaptive responses to $\mathrm{CS}+$.

Row ii: A DAN selective to US that acquires a CS+ response after conditioning.

Row iii and iv: DANs with "prediction-error" like responses. CS+

Feedback neurons enable adaptive responses of modulatory neurons in the model

The high fraction of feedback input originating from MBONs onto modulatory neurons suggests that their activity could be adaptively regulated by prior learning. To test this idea, we computed an index that quantifies the mean change in modulatory neuron firing rates in response to CS+ (i.e. the CS that was paired with the US) presentations before and after conditioning in the model and found that it is indeed substantially enhanced by the presence of feedback neurons (Fig. 7c). The optimized networks exhibit a diversity of adaptive modulatory neuron responses (some examples are shown in Fig. 7d).

After a CS/US pairing, many modulatory neurons acquired responses to $\mathrm{CS}+$ that resemble their responses to the US that had been paired with that CS+ (Fig. $7 \mathrm{e}$ and $7 \mathrm{~d}-\mathrm{ii})$. These responses were significantly attenuated in networks that lacked feedback, including those that lacked just indirect feedback, and just crosscompartment feedback (Fig. 7e). Such responses have been observed in modulatory neurons of species across the animal kingdom ${ }^{12,23,32}$, including adult and larval Drosophila ${ }^{43,44,144}$. They are consistent with a computa-

responses are opposite in sign to US responses.

Row iii: a neuron that is inhibited by the US is activated when that US is omitted (e.g. when the CS+ is presented alone after training). Row iv: a neuron that is excited by the US is inhibited when that US is omitted (e.g. when the CS+ is presented alone after training). Note that the negative $\mathrm{CS}+$ response is prolonged compared to the response to $\mathrm{CS}$ prior to training.

e CS+/US response overlap before and after conditioning. The overlap is computed using the dot product of the vectors of firing rate changes across the modulatory neuron population during $\mathrm{CS}+$ and US presentations. $p$-values represent a comparison to control networks, after conditioning. tion of the valence of the US that is predicted by the CS+ (i.e. a predicted value associated with the $\mathrm{CS}+$ ) and could drive the formation of an association between a novel CS and a CS+ during higher-order conditioning.

Additionally, some modulatory neurons acquired CS+ responses that were opposite in sign to their responses to that US (Fig. 7d-iii and 7d-iv). Some of those appear to be activated by the omission of a predicted US whose valence is opposite that of neuron's preferred US (Fig. 7d-iii). Such responses have been proposed to support extinction by inducing a parallel memory of opposite valence following US omission ${ }^{9,13,14,53}$. Consistent with this idea, in adult flies, DANs of opposite valence and direct cross-compartment MBON-to-DAN connections have been implicated in extinction ${ }^{13,14,53}$, but the role of indirect feedback pathways has not been investigated. In our model we find that removing indirect feedback significantly reduces performance of networks optimized to extinguish a previous association (Fig. 7a). Some modulatory neurons also showed prolonged inhibition in response to the omission of a predicted US whose valence is the same as the neuron's preferred US (Fig. 7d-iv). Such responses have been proposed to support extinction 
in mammals by erasing the memory formed by the activation of that modulatory neuron ${ }^{9,28,145}$. Thus, our model raises the possibility that extinction could be implemented via multiple mechanisms in this circuit ${ }^{9}$.

\section{Discussion}

Modulatory neurons (e.g. dopaminergic, DAN) are key components of higher-order circuits for adaptive behavioral control, such as the vertebrate basal ganglia or the insect mushroom body (MB), and they provide teaching signals that drive memory formation and updating ${ }^{12,14,21,24,25,49,58,66}$. Here, we provide the first synaptic-resolution connectivity map of a recurrent neural network that regulates the activity of all modulatory neurons in a higher-order learning center, the Drosophila larval MB (Fig. 2a-f). We discovered an unexpected component of the insect MB: a large population of 61 feedback neuron pairs that provide one- and/or two-step feedback from the MB output neurons (MBONs) to modulatory neurons (Fig. 2a-d, 3a-b and 5a-e). The majority of these one- and two-step feedback pathways link distinct memory systems, suggesting that the entire MB functions as an interconnected ensemble during learning (Fig. 3b and 5e). We also systematically determined which modulatory neurons evoke aversive and appetitive memories and functionally tested some of the newly identified structural pathways (Fig. 1c-f, 4b-g, and 6a-f). We developed a model of the circuit constrained by the connectome and by our functional data and explored the roles of the newly discovered architectural motifs in different learning tasks (Fig. 7a-e). Our study provides a basis for understanding the circuit implementation of learning algorithms in the tractable insect nervous system.

\section{Feedback pathways enable adaptive regulation of learning by prior learning}

Adaptive regulation of modulatory neuron activity has been proposed to underlie aspects of learning and memory in both vertebrates ${ }^{31,32,146,147}$ and insects ${ }^{23,42-44}$, including long-term memory consolidation $46,49,100,148$, memory reconsolidation ${ }^{11,13,14}$, extinction ${ }^{13,14,53,149}$, and higherorder conditioning 21,150 . However, the architecture and functional principles of the circuits that support this regulation are not well understood. Furthermore, the extent to which modulatory neuron activity is regulated by previous memories in insects is still unclear. Strikingly, we found that many modulatory neurons, including the ones that provide teaching signals for appetitive and aversive olfactory memory formation (Fig. 1c), receive more than $50 \%$ of their total dendritic input from feedback pathways that relay MBON signals (Fig. 2d). We confirmed that some of the identified indirect feedback pathways are functional and that feedback neurons can induce memory formation (Fig. 4b-g and 6a-f). These results suggest that prior memories as represented by the pattern of MBON activity can strongly influence modulatory neuron activity in Drosophila larva. Indeed, in our connectivity-constrained model, modulatory neuron responses to CS are modified after pairing with US, and this modulation is reduced in the absence of feedback neurons (Fig. 7c-e). While further model constraints are likely required to directly compare model neurons to recordings, the model demonstrates that feedback supports such adaptive responses.

Learning and memory systems in vertebrates ${ }^{151,152}$ and insects ${ }^{45,47,48,55,84,94,153}$ are often organized into distinct compartments implicated in forming distinct types of memories (e.g. aversive and appetitive or short- and long-term). However, the extent and nature of interactions between distinct memory systems during memory formation is still an open question. Here, we provide the first comprehensive view of an extensive set of anatomical feedback pathways that can mediate interactions between distinct memory systems (Fig. 3b-c, 5e, and Extended Data Fig. 9b). The cross-compartment feedback pathways identified here suggest that prior memories formed about an odor in one compartment can influence the formation and updating of future memories about that odor in many other functionally distinct compartments.

Interestingly, we also found that the feedback neurons receive input from brain areas other than MB (Extended Data Fig. 4a-c). They could therefore play a role in encoding variables determined by these areas, such as context or internal state. Consistent with this, our model revealed that the performance on a context-dependent conditioning task was significantly reduced in the absence of these neurons (Fig. 7b). The feedback neurons could therefore provide a substrate for flexible and adaptive regulation of learning, based on both previous experience and context or internal state.

\section{Circuit motifs for computing integrated predicted value signals across aversive and appetitive mem- ory systems}

The use of internal predictions to inform future learning can dramatically increase the flexibility of a learning system ${ }^{28,29}$. Indeed, modulatory neurons in vertebrates ${ }^{12,21,32}$ and insects ${ }^{23}$, including adult and larval Drosophila ${ }^{43,44,144}$, show adaptive responses consistent with the idea that they encode predictions. However, the circuit properties that permit the computation of predictions are not well understood. In particular, the way in which integrated common-currency predicted value signals across appetitive and aversive memory systems are computed is unclear. Our study reveals candidate circuit motifs that could implement this computation and mediate more complex tasks that require it, such as second-order conditioning. While second-order conditioning has not yet been investigated in the larva, adult Drosophila has been shown to be capable of $i^{20}$.

A prominent motif revealed by our analysis of connectivity and neurotransmitter expression is convergence of potentially excitatory and inhibitory connections from MBONs from compartments of opposite valence onto some DANs (Fig. 3b-e). We confirmed that DAN-i1 (whose activation can induce appetitive memory for paired odor) receives functionally inhibitory input from its own compartment and functionally excitatory (likely disinhibitory) input from compartments of opposite valence profile (Fig. 4a-g). By integrating these inputs, such DANs may compute a comparison between the odor-evoked excitation of MBONs in compartments of opposite valence. In naïve animals, odor-evoked MBON excitation in all compartments 
is thought to be similar. However, associative learning selectively depresses conditioned odor MBON excitation in compartments whose modulatory neuron activation has been paired with the odor ${ }^{14,66,85,90,133}$. The valence predicted by the conditioned odor is therefore thought to be encoded as a skew in the relative excitation of MBONs in compartments of opposite valence. We propose that by comparing the conditioned odor-evoked MBON excitation in compartments of opposite valence via the crosscompartment feedback connections, modulatory neurons could compute an integrated common-currency predicted value signal across appetitive and aversive domains. Our model results are consistent with this idea. Some modulatory neurons in the model acquire responses to conditioned odors that resemble their responses to US (Fig. 7dii and 7e), consistent with a value prediction, and these responses, as well as performance on second-order conditioning, were degraded in the absence of cross-compartment feedback (Fig. 7a and 7e).

\section{Convergence of feedback and US pathways could allow the computation of prediction errors}

An important aspect of reinforcement learning theories is the idea that modulatory neurons compare predicted and actual US (compute the so-called prediction errors) and drive memory formation or extinction depending on the sign of the prediction error. Computing prediction errors requires structural convergence between feedback pathways, which carry information about predicted US valence, and afferent US pathways. However, the site of this convergence is still an open question. Feedback and afferent US pathways could converge at the modulatory neurons themselves, upstream of modulatory neurons, or both.

While Drosophila modulatory neurons have not yet been directly shown to represent prediction errors, adult and larval Drosophila are capable of extinction $^{5,13,53,141,154}$, and our study reveals candidate motifs that could support the comparison of expected and actual US. We found that modulatory neurons receive convergent input from feedback pathways from MBONs and from US pathways (Fig. 2d-f). Modulatory neurons could therefore potentially compute prediction errors by comparing inhibitory drive from the feedback pathways to the excitatory drive from the US pathways, or vice versa, excitatory drive from the feedback pathways and inhibitory drive from the US pathways. Consistent with this idea, we observed in our model some DANs that are inhibited by US alone and activated by CS+ alone, or vice versa (Fig. 7d-iii and 7d-iv).

Our study also revealed that US pathways and feedback pathways converge at two levels: not only at the modulatory neurons themselves, but also at the two-step feedback neurons (FB2Ns, Fig. 2f). Actual and expected outcomes could therefore also be compared by FB2Ns. A recent study in the mouse VTA used retrograde labelling and electrophysiology to characterize the response properties of some of the neurons presynaptic to the DANs ${ }^{12,41}$ and found that a fraction of the analyzed pre-DAN neurons encoded only actual, or only expected reward, while the remainder encoded both variables ${ }^{41}$. Thus, both in vertebrates and in insects, comparing predicted and actual outcomes may be a complex computation involving multiple levels of integration that eventually converges onto an ensemble of modulatory neurons ${ }^{41}$. The connectome of such a network presented here provides a basis for understanding the circuit implementation of this computation.

\section{The multilevel and cross-compartment feedback increase performance and flexibility}

Our connectivity and modeling studies revealed two architectural features of the circuit that provides input to the modulatory neurons that increase its computational performance and flexibility on learning tasks (Fig. 7a-b). The first is the multilevel feedback architecture that includes not only the previously known direct MBON feedback, but also multiple levels of indirect feedback. The second is the extensive set of cross-compartment connections. Our results also reveal modulatory neurons receive a diverse set of feedback inputs (Fig. 2e) that could enable each modulatory neuron to compute a unique set of features. Consistent with this, we observed a diversity of adaptive response types in the modulatory neurons in our model. This suggests that instead of computing a single global reward prediction error that is distributed to all modulatory neurons ${ }^{21}$, the network uses a range of distinct compartmentalized and distributed teaching signals.

In adult Drosophila, functional connections between some MBONs and DANs ${ }^{13,46,49,53-55,153}$, as well as between KCs and $\mathrm{DANs}^{48,155}$ have been reported, and some of these have been shown to play a role in short-term memory formation ${ }^{49,153}$, long-term memory consolidation $^{46,54}$, re-consolidation ${ }^{13}$, extinction ${ }^{53}$, or in synchronizing DAN ensemble activity in a contextdependent manner ${ }^{55}$. For some of these cases, direct within- or cross-compartment MBON-to-DAN connections have been demonstrated ${ }^{13,46,49}$. While direct axodendritic connections from several MBONs onto DANs exist in the larva ${ }^{47}$ (Extended Data Fig. 9a), we find that indirect connections via the feedback layer account for a much larger fraction of a modulatory neuron's dendritic input than direct MBON synapses (Fig. 2d), suggesting that adaptive DAN responses may be largely driven by such indirect feedback. Additionally, direct axo-axonic connections from KCs could modulate modulatory neuron output in both larva ${ }^{47}$ and adult ${ }^{48,155}$, but they cannot convey learning-related changes in the strengths of KC-to-MBON connections, so they likely play a very different role to the feedback from MBONs. Since many aspects of connectivity between the core components of the MB (modulatory neurons, KCs and MBONs) are shared between larval and adult Drosophila stages and other insects ${ }^{47,48,67}$, we expect that the indirect feedback motifs discovered here are also shared across insects.

Some of the within-compartment feedback motifs we found are reminiscent of the feedback motifs so far described for the DANs in the vertebrate midbrain. Rabies tracing studies have shown that DANs receive input from their direct targets in the striatum (analogous to direct MBON to DAN connections), as well as from the direct targets of striatal neurons (analogous to the one-step feedback described here $)^{12,39,41,50}$. While the diversity and the inputs of striatal feedback neurons have not yet been fully explored, in the future it will be interesting to determine 
whether many of the striatal feedback neurons also link distinct memory systems.

In summary, we present the first complete circuit diagram of a recurrent network that computes teaching signals in a biological system, providing insights into the architectural motifs that increase the computational power and flexibility of the learning center. Our connectomeconstrained model provides numerous predictions that can be tested in the future in a tractable model organism, for which genetic tools can be generated to monitor and manipulate individual neurons ${ }^{102,134,156,157}$. The connectome, together with the functional and modelling studies therefore provides exciting opportunities for elucidating the biological implementation of reinforcement learning algorithms.

\section{Acknowledgements}

We thank Fly Light at HHMI Janelia Research Campus (JRC) for generating confocal images of the GAL4 lines, J. Simpson for sharing a 2-photon microscope, Y. Aso and G. Rubin, for sharing unpublished driver lines, V. Jayaraman for sharing unpublished versions of CsChrimson and GCamp6f, L. Feng and I. Andrade for help with circuit mapping from EM, K. Hibbard and JRC FLY Core, for generating some of the fly stocks, Fly EM at JRC for generating the EM volume, T. Saumweber for discussions, D. Bonnery for help with analyses, L. Abbott for helpful comments on the manuscript, Z. Zavala-Ruiz and the JRC Visiting Scientist Program and HHMI JRC for funding. A.L.-K. was supported by the Burroughs Wellcome Foundation, the Gatsby Charitable Foundation, the Simons Collaboration on the Global Brain, and NSF award DBI1707398. B.G. received grant support from the Deutsche Forschungsgemeinschaft Ge1091/4-1 and FOR 2705-TP2.

\section{Materials and Methods}

\section{Fly lines}

In the main text and figures, short names are used to describe genotypes for clarity. We used GAL4, Split-GAL4 lines to direct the expression of the red-shifted channelrhodopsin 20XUAS-CsChrimson-mVenus ${ }^{103}$ (Bloomington Drosophila Stock Center BDSC 55134, gift of $\mathrm{V}$. Jayaraman) or the Calcium indicator 20xUAS-IVSGCaMP $6 f^{127}$ in pairs of neurons or subsets of neurons. Split-GAL4 lines restrict expression of the effector to a few cells, under the double control of two enhancers (inserted in the attP2 and attP40 docking sites), selected by us or others in Janelia Research Campus (HHMI, VA, USA) based on their GAL4 expression pattern ${ }^{101,102,158}$.

\section{Modulatory MBINs}

We used SS24765-Split-GAL4 to optogenetically activate OAN-a1 in the calyx. We generated SS02160-Split-GAL4 to activate DAN-c1 in the lower peduncle. For the vertical lobe, we generated SS01702-Split-GAL4 to activate or image calcium transients in MBIN-e2 (DAN-c1 was also covered by this line) and SS01958-Split-GAL4 to activate or image calcium transients in OAN-e1 in the UVL.
We used SS02180-Split-GAL4, MB145B-Split-GAL4 (used for activation and calcium imaging, gift of G. Rubin and Y. Aso) and $M B 065 B-S p l i t-G A L 4^{45}$ (which also covered DAN-c1) to target DAN-f1 in the IVL. We used $S S 01716$ Split-GAL $4^{58}$ to induce or image DAN-g1 activity in the LVL, and we generated SS04268-Split-GAL4 to activate OAN-g1, also in the LVL. MB054B-Split-GAL4 (gift of G. Rubin and Y. Aso) was also used to co-activate DANg1 and DAN-f1. We used two lines to target DAN-d1 in the lateral appendix: MB143B-Split-GAL4 (used for activation and calcium imaging) and MB328B-Split-GAL4 (both gifts of G. Rubin and Y. Aso). In the medial lobe, we generated a broad line SS01948-GAL4 which allows coactivation of DAN-h1, DAN-i1, DAN-k1, and sometimes DAN-j1. We also imaged calcium transients in DAN-i17 using the more specific GAL4 SS00864-Split-GAL4.

\section{Neurons presynaptic to the modulatory MBINs}

We optogenetically activated multidendritic Class IV neurons (MD IV) with the driver line $p p k-1.9-G A L 48$ (gift of D. Tracey); Basin interneurons with GMR72F11-GAL4 $4^{62}$; the ascending neuron A00c with GMR71A10-GAL $4^{62,123}$ crossed to $p p k-G A L 80^{160}$, repo-GAL80 $0^{161}$ (to prevent expression in MD IV and glial cells, respectively). We also activated A00c with the more specific GAL4 line SS00883-Split-GAL4. We generated SS01778-Split-GAL4 and SS02181-Split-GAL4, which target FB2N-11 and/or FB2N-18. SS02108-Split-GAL4 targets FAN-7; SS02401Split-GAL4 targets FB2N-19.

\section{Control lines}

As a control for the GAL4 lines inserted at the attP2 site, we used the empty control stock y $w ; ; a t t P 2^{102,158}$ crossed to the effector line. As a control for Split-GAL4 lines with $\mathrm{AD}$ at $a t t P 40$ and DBD at attP2, we used the empty stock y w;attP40;attP2 $2^{102,158}$ crossed to the effector line.

\section{Lines for recording neuronal activity}

Calcium transients in modulatory neurons were imaged using the following constructs to verify functional input of mechano-ch neurons: $w$; iav-LexA ${ }^{62}$ in attP40; 20xUAS-IVS-GCaMP6f $15.693^{127}$ in attP2, 13XLexAop2CsChrimson-tdTomato ${ }^{103}$ in VK00005. For Basins multisensory interneurons: $w$; GMR72F11-LexA $A^{158}$ in JK22C; 20xUAS-IVS-GCaMP6f $15.693^{127}$ at attP2, 13XLexAop2CsChrimson-tdTomato ${ }^{103}$ at VK00005. And for MD class IV nociceptive neurons: $w$; 13XLexAop2-CsChrimson$m$ Venus $^{103}$ at attP40 (BDSC 55138); ppk-1kb-hs43-lexAGAD10 at attP2, 20xUAS-IVS-GCaMP6f $f^{127}$ at VK00005. All the effectors used in these stocks are a gift from V. Jayaraman. Transvection was tested by bathing some samples in $100 \mathrm{mM}$ mecamylamine and observing the disappearance of responses to optogenetic stimulation (data not shown). If a response remained during mecamylamine application, the experiments were repeated using a spatially defined photo-stimulation using spatial light modulator (SLM) technology (see functional connectivity section for details of the procedure and the lines concerned).

For patch-clamp recording we crossed the genetic driver lines for MBON-m1 (SS02163-Split-GAL4) or for MBON-i1 (SS01726-Split-GAL4) to 58E02-LexAp65 at 
attP40 $48 ; \quad$ 13xLexAop2-IVS-GCaMP6f-p10 $15.693^{127}$ at VK00005 (BDSC 44276), 20xUAS-CsChrimson$m$ Cherry $^{103}$ at $s u(H w) a t t P 1$ in order to activate MBONs and visualize the medial lobe DANs (ML-DANs) for patch-clamping. Only data for DAN-i1, which was the most frequently hit by the recording pipette, as revealed by post hoc identification, are shown.

The reporter pJFRC29-10xUAS-IVS-myr::GFP$p 10^{135}$ at attP2 was used for immunostaining.

\section{Learning experiments}

Learning experiments were performed as previously described ${ }^{58,47,56}$. The larvae were reared in the dark at $25^{\circ} \mathrm{C}$ in food vials supplemented with 1:200 retinal. The experimenter selected two groups of 30 third-instar larvae and was blind to their specific genotype. The two groups underwent a training procedure involving odor and light exposures, either fully overlapping in time (paired group), or fully non-overlapping (unpaired group). The paired group was placed for 3 minutes on $4 \%$ agarose plates and exposed to constant red-light illumination (wavelength: $629 \mathrm{~nm}$, power: $350 \mu \mathrm{W} / \mathrm{cm} 2$; except for $p p k-1.9-G A L 4$, which targets neurons at the surface of the body and for which a light power of $35 \mu \mathrm{W} / \mathrm{cm} 2$ was used) paired with the presentation of $12 \mu \mathrm{l}$ of odor ethyl-acetate $\left(10^{-4}\right.$ dilution in distilled water) absorbed on two filter papers located on the plate lid. These larvae were then transferred to a new plate with no odor and in the dark for 3 minutes. This paired training cycle was repeated three times in total. The unpaired group of larvae underwent odor presentation in the dark and red light without odor following the same protocol. After a 3-minute test with odor presentation on one side of the plate lid, larvae were counted on the side of the odor, on the opposite side, and in the $1 \mathrm{~cm}$-wide midline of the plate. Preference and performance indices were calculated as in a previous study ${ }^{57}$. Briefly, a preference index (PI) was first computed, for each group as: $\mathrm{PI}=[\mathrm{N}$ (larvae on the odor side) - N (larvae on the no-odor side)]/N(total), N(total) includes larvae in the middle of the plate. The Learning Performance Score (LPS) was then computed as LPS $=[\mathrm{PI}$ (paired) PI (unpaired)] $/ 2$.

\section{Circuit mapping and electron microscopy}

We reconstructed neurons and annotated synapses in a single, complete central nervous system from a $6 \mathrm{hr}$ old female [iso] Canton S G1 x w1118 [iso] 5905 larva, acquired with serial section transmission EM at a resolution of $3.8 \times 3.8 \times 50 \mathrm{~nm}$, that was first published along with the detailed sample preparation protocol ${ }^{62}$. Briefly, the CNS was dissected and placed in $2 \%$ gluteraldehyde 0.1 $\mathrm{M}$ sodium cacodylate buffer ( $\mathrm{pH}$ 7.4). An equal volume of $2 \% \mathrm{OsO}_{4}$ was added and the larva was fixed with a Pelco BioWave microwave oven with $350-\mathrm{W}, 375-\mathrm{W}$ and $400-\mathrm{W}$ pulses for $30 \mathrm{sec}$ each, separated by 60 -sec pauses, and followed by another round of microwaving but with $1 \% \mathrm{OsO}_{4}$ solution in the same buffer. Next, samples were stained en bloc with $1 \%$ uranyl acetate in water and microwaved at $350 \mathrm{~W}$ for $3 \times 330 \mathrm{sec}$ with $60-\mathrm{sec}$ pauses. Samples were dehydrated in an ethanol series, transferred to propylene oxide, and infiltrated and embedded with Epon resin. After sectioning the volume with a Leica UC6 ultramicrotome, sections were imaged semiautomatically with Leginon ${ }^{162}$ driving an FEI Spirit TEM (Hillsboro, OR), and then assembled with TrakEM2 $2^{163}$ using the elastic method ${ }^{164}$. The volume is available at https://1em.catmaid.virtualflybrain.org/?pid=1.

To map the wiring diagram we used the web-based software CATMAID ${ }^{165}$, updated with a novel suite of neuron skeletonization and analysis tools ${ }^{128}$, and applied the iterative reconstruction method ${ }^{128}$. All annotated synapses in this wiring diagram fulfill the four following criteria of mature synapses ${ }^{62,128}$ : (1) There is a clearly visible T-bar or ribbon on at least two adjacent z-sections. (2) There are multiple vesicles immediately adjacent to the T-bar or ribbon. (3) There is a cleft between the presynaptic and the postsynaptic neurites, visible as a dark-light-dark parallel line. (4) There are postsynaptic densities, visible as dark staining at the cytoplasmic side of the postsynaptic membrane.

We validated the reconstructions as previously described $^{62,128}$, a method successfully employed in multiple studies ${ }^{62,124,128,132,166,167}$. Briefly, in Drosophila, as in other insects, the gross morphology of many neurons is stereotyped and individual neurons are uniquely identifiable based on morphology ${ }^{167-169}$. Furthermore, the nervous system in insects is largely bilaterally symmetric and homologous, with mirror-symmetric neurons reproducibly found on the left and the right side of the animal. We therefore validated neuron reconstructions by independently reconstructing synaptic partners of homologous neurons on the left and right side of the nervous system. With this approach, we have previously estimated the false positive rate of synaptic contact detection to be 0.0167 ( 1 error per 60 synaptic contacts $)^{56}$. Assuming the false positives are uncorrelated, for an n-synapse connection the probability that all $\mathrm{n}$ are wrong (and thus that the entire connection is a false positive) occurs at a rate of $0.0167^{n}$. Thus, the probability that a connection is a false positive reduces dramatically with the number of synaptic contacts contributing to that connection. Even for $n=2$ synaptic contacts, the probability that a connection is not true is 0.00028 (once in every 3,586 two-synapse connections). We therefore consider 'reliable' connections those for which the connections between the left and right homologous neurons have at least 3 synapses each and their sum is at least 10 . See $\mathrm{e}^{62,128}$ for more details.

\section{Immunostaining}

Dissected brains were fixed in phosphate buffered saline (PBS, NaCl $137 \mathrm{mM}, \mathrm{KCl} 2.7 \mathrm{mM}, \mathrm{Na}_{2} \mathrm{HPO}_{4} 8.1 \mathrm{mM}$, KH2PO4 $1.5 \mathrm{mM}$, pH7.3) containing $4 \%$ paraformaldehyde (Merck) for 30-min at room temperature. After two 15 -minute washes with PBT (PBS with $1 \%$ or $3 \%$ Triton X-100; Sigma-Aldrich), the brains were blocked with $5 \%$ normal goat serum (Vector Laboratories) in PBT and incubated for at least 24 hours with primary antibodies at $4^{\circ} \mathrm{C}$. Before application of the secondary antibodies for at least 24 hours at $4^{\circ} \mathrm{C}$ or for 2 hours at room temperature, brains were washed several times with PBT. After that, brains were again washed with PBT, mounted in Vectashield (Vector Laboratories) and stored at $4^{\circ} \mathrm{C}$ in darkness. Images were taken with a Zeiss LSM 710M confocal microscope. The resulting image stacks were 
projected and analyzed with the image processing software Fiji ${ }^{170}$. Primary antibodies were used at the following dilutions: rabbit anti-GFP (cat\# Af2020, Frontier Institute; 1:1000), chick anti-GFP (ab13970, abcam, 1:1000), rabbit anti-GABA (A2052, Sigma; 1:100), mouse anti-ChAT (ChAT4B1, DSHB Hybridoma Product deposited by P.M. Salvaterra; 1:50). Rabbit anti-DVGlut ${ }^{137}$ was diluted 1:1000. Secondary antibodies were used at the following dilutions: Alexa Fluor 568-conjugated goat anti-rabbit IgG (A-11036, Invitrogen Molecular Probes; 1:300), Alexa Fluor 633-conjugated goat anti-mouse IgG (A-21050, Invitrogen Molecular Probes; 1:300) and Alexa Fluor 488-conjugated goat anti-chicken IgG (A-11039, Invitrogen Molecular Probes; 1:300).

\section{Identifying GAL4 lines that drive expression in modulatory neurons and their presynaptic part- ners}

To identify GAL4 lines (listed in Supplementary Table 1) that drive expression in specific neurons, we performed single-cell FlpOut experiments (for FlpOut methodology see ${ }^{62,171}$ ) of many candidate GAL4 lines ${ }^{134}$. We generated high-resolution confocal image stacks of individual neuron morphology (multiple examples per cell type). Most MBONs and MBINs were uniquely identifiable based on the dendritic and axonal projection patterns (which MB compartment they project to and the shape of input or output arbor outside the MB). These were also compared to previously reported singlecell FlpOuts of dopaminergic and octopaminergic neurons in the larva $57,95,123,172,173$. For the neurons upstream of MBINs (FBNs/FANs/FB2Ns), we used morphology and cell body position to identify the lineage of the neuron. The precise shape and 3D location of dendritic and axonal projections were then examined and compared to all potential candidates in the lineage which have been fully reconstructed from the electron microscopy volume. In some cases, two neurons had very similar morphology at both light and EM level, and in such cases they also had nearly identical connectivity (e.g. FB2N-11 and FB2N18).

\section{Functional connectivity assays}

Central nervous systems were dissected in a cold buffer containing $103 \mathrm{mM} \mathrm{NaCl}, 3 \mathrm{mM} \mathrm{KCl}, 5 \mathrm{mM}$ TES, 26 $\mathrm{mM} \mathrm{NaHCO} 3,1 \mathrm{mM} \mathrm{NaH} \mathrm{PO}_{4}, 8 \mathrm{mM}$ trehalose, $10 \mathrm{mM}$ glucose, $2 \mathrm{mM} \mathrm{CaCl}_{2}, 4 \mathrm{mM} \mathrm{MgCl}_{2}$ and adhered to poly-Llysine (SIGMA, P1524) coated cover glass in small Sylgard (Dow Corning) plates.

For optogenetic activation, red illumination $(617 \mathrm{~nm}$ High-Power Lightguide Coupled LED Source, Mightex) was positioned above the sample to depolarize the axon terminal parts of the sensory neurons (MD IV or chordotonal) or the second order interneurons (Basins). Light stimulations were performed with 1 or 15 sec duration and in 40 and 600 cycles of laser on/off pulses of $20 \mathrm{msec} / 5$ msec. Each preparation underwent three types of light stimulation of increasing power: ca. $390 \mu \mathrm{W} / \mathrm{mm}^{2}, 920$ $\mu \mathrm{W} / \mathrm{mm}^{2}$ and $4.6 \mathrm{~mW} / \mathrm{mm}^{2}$. Only the data for the highest light power during $1 \mathrm{sec}$ is displayed (Fig.1f). The same stimulus was spaced with $30 \mathrm{sec}$ for a total of three presentations in each scan. Each scan consisted in imaging dopaminergic neurons on a two-photon scanning microscope (Bruker) using a 60x 31.1 NA objective (Olympus). A mode-locked Ti:Sapphire laser (Chameleon Ultra II, Coherent) tuned to $925 \mathrm{~nm}$ was used for photo-activation of the GCaMP6f. Fluorescence was collected with photomultiplier tubes (Hamamatsu) after band-pass filtering. Images were acquired in line scanning mode ( $5.15 \mathrm{fps}$ ) for a single plane of the CNS.

To overcome transvection observed between the transgenes at the attP40 landing site of the MB143B-SplitGAL4 line (targeting DAN-d1) crossed to w; 13XLexAop2CsChrimson-mVenus ${ }^{103}$ in attP40; ppk-1kb-hs43-lexAGAD10 in attP2, 20xUAS-IVS-GCaMP6f2 in VK00005, we used 3-dimension spatially defined photo-stimulation. MD IV neurons expressing CsChrimson were photoactivated by a holographic pattern generated by a twophoton $1040 \mathrm{~nm}$ laser (femtoTrain, Spectra-Physics) coupled to a phase-only SLM (Intelligent Imaging Innovations). GCaMP6f signal was imaged by a laser tuned to $925 \mathrm{~nm}$ (Insight DS+ Dual, Spectra-Physics). The optogenetic stimulations were 50 cycles of laser on/off pulses of 2 $\mathrm{msec} / 18 \mathrm{msec}$, ranging from 1 to $1.5 \mathrm{~mW} / \mathrm{mm}^{2}$. Off-target (equidistant from the Chrimson-expressing DAN-d1 neuron, but not targeting Chrimson-expressing MD IV neurons) and on-target stimulations were alternatively performed and the difference between transvection-only generated calcium signals and transvection + MD IV neuron activation-generated signal was computed and used as the fluorescence signal. DAN-d1 neurons were imaged at a frame rate of ca. $5 \mathrm{fps}$ on a two-photon scanning microscope (Vivo, Intelligent Imaging Innovations) using a 25x 2 1.1 NA objective (Nikon).

For image analysis, image data were processed by Fiji software $^{170}$ and analyzed using custom code in Matlab (The Mathworks, Inc). Specifically, we manually determine the regions of interest (ROIs) from maximum intensity projection of entire time series images, and measure the mean intensity. In all cases, changes in fluorescence were calculated relative to baseline fluorescence levels (F0) as determined by averaging over a period of at least 2 sec. just before the optogenetic stimulation. The $\delta \mathrm{F} / \mathrm{F}_{0}$ values were calculated as $\delta \mathrm{F} / \mathrm{F}_{0}=\left(\mathrm{F}_{t}-\mathrm{F}_{0}\right) / \mathrm{F}_{0}$, where $\mathrm{F}_{t}$ is the fluorescent mean value of a ROI in a given frame. Analyses were performed on the mean $\delta \mathrm{F} / \mathrm{F}_{0}$ of the consecutive 3 stimulations.

\section{Whole-cell patch-clamp recordings from DANs on optogenetic activation of MBONs}

For recording, the isolated brain attached with VNC were dissected from third instar larvae in Baines external solution ${ }^{174}$, which contained $(\mathrm{mM}): 135 \mathrm{NaCl}, 5$ $\mathrm{KCl},, 2 \mathrm{CaCl}_{2} .2 \mathrm{H}_{2} \mathrm{O}, 4 \mathrm{MgCl}_{2} \cdot 6 \mathrm{H}_{2} \mathrm{O}, 5$ 2-[(2-Hydroxy1,1-bis(hydroxymethyl)ethyl)amino] ethanesulfonic acid, $5 \mathrm{~N}$-[Tris(hydroxymethyl) methyl]-2-aminoethanesulfonic acid, and 36 sucrose. The pH was adjusted to 7.15 with $\mathrm{NaOH}$, and osmolarity was $310-320 \mathrm{mOsm}$. The preparation was viewed with a $60 \mathrm{x} 1 \mathrm{NA}$ water-immersion objective equipped with an Olympus microscopy (BX51WI; Olympus). GCaMP6f-labeled DANs were visualized with a 470-nm wavelength LED. The glial sheath above the targeted DANs was ruptured using $0.1 \%$ protease (Protease XIV; Sigma-Aldrich). Recording elec- 
trodes were pulled from thick-wall glass pipet (O.D. $1.5 \mathrm{~mm}$, I.D. $0.86 \mathrm{~mm}$ ) using P-97 puller (Sutter Instruments) and fire-polished to resistances of 10-15 $\mathrm{M} \omega$. The Baines intracellular solution ${ }^{174}$ contained $(\mathrm{mM}): 140$ potassium gluconate, $5 \mathrm{KCl}, 2 \mathrm{MgCl}_{2} \cdot 6 \mathrm{H}_{2} \mathrm{O}, 2 \mathrm{EGTA}, 20$ HEPES. The $\mathrm{pH}$ was adjusted to 7.4 with $\mathrm{KOH}$, and the osmolarity was $280 \mathrm{mOsm}$. Biocytin was dissolved in intracellular solution at $0.5 \%$ for further post hoc morphological identification of recorded DANs. The data were acquired and processed using Digidata 1550, Multiclamp 700B, and Clampex 10.4 software (Molecular Devices). The recording was sampled at $20 \mathrm{kHz}$ and filtered at $6 \mathrm{kHz}$ under current-clamp mode. CsChrimson was activated by 617-nm wavelength LED.

DAN identification: After the electrophysiology recording, the preparation containing the $\mathrm{VNC}$ and brain was fixed in $4 \%$ paraformaldehyde in $0.1 \mathrm{M}$ phosphate buffer saline (PBS) overnight at $4^{\circ} \mathrm{C}$, and then transferred to PBS until staining. After rinsing in PBS, the CNS preparations were placed in Streptavidin Alexa Fluor 647 (1:200) in PBS with 10\% Triton X (overnight, room temperature). After rinsing, the preparations were dehydrated and mounted with DPX. The confocal images were captured with Zeiss 800 confocal laser microscope. Alexa Fluor 647 was excited with $633 \mathrm{~nm}$-wavelength light, and mCherry-tagged CsChrimson neurons were excited with 567 nm-wavelength light.

\section{Statistical analysis}

As most fluorescence and behavioral data were nonnormally distributed (according to a Shapiro-Wilk test), we opted for non-parametric tests for paired comparisons.

For behavioral experiments, the performance scores obtained for each line tested in optogenetic reinforcement were compared to the ones of its corresponding empty line (i.e. w;;attP2 or w;attP40;attP2 for GAL4 or Split GAL4, respectively) using a non-parametric Mann-Whitney U test for independent sets of data. For multiple comparisons, the probability values were compared to a threshold of 0.05 adjusted with a HolmBonferroni correction to balance for Type I and Type II statistical errors, unless otherwise stated. Across GAL4 lines, comparisons of performance scores were done using the same methodology. Data were plotted using the Matlab script errorbarjitter, available at http://www.mathworks.com/matlabcentral/fileexchange/ 33658-errorbarjitter.

Fluorescence analyses were done using a nonparametric Wilcoxon test for paired comparisons between the maximum $\delta \mathrm{F} / \mathrm{F}_{0}$ plus one standard deviation during $1 \mathrm{sec}$ before photostimulation onset and the maximum $\delta \mathrm{F} / \mathrm{F}_{0}$ at two time windows: during the 1 sec of the stimulation, and from 1 to 3 seconds after its onset.

For the clustering analysis, we looked for clusters among FBNs/FANs based on the similarity of their synaptic partners separately for input and output. To find clusters based on synaptic inputs, we defined the similarity between a pair of FBN/FANs as the cosine similarity of the vector of inputs they receive from MBONs where the weight of a given connection is measured as the fraction of total input synapses on the postsynaptic neuron. Specifically, for $v_{i}$ and $v_{j}$ being the input vectors for FBN/FANs $i$ and $j$, the similarity between them is defined $S_{i j}=\frac{v_{i} \cdot v_{j}}{\left\|v_{i}\right\|\left\|v_{j}\right\|}$. Hierarchical clustering on the similarity matrix was done with Scipy using average linkage. We chose the top five clusters to highlight, which included all clearly differentiated groups of FBN/FANs. Clustering on the output patterns was done identically using the vectors of connectivity from FBN/FANs onto MBINs.

For the input-clustered groups, we assessed the similarity of the patterns of synaptic outputs and vice versa for the synaptic input patterns for output-clustered groups. We measured the overall group similarity as the median of all unique pairwise cosine similarities between neurons within the group. We used a permutation test to assess the significance of the observed similarities by randomizing the relationship between input pattern and output pattern for each FAN/FBN. For example, for each input-clustered group of size $n$, we randomly chose $n$ output patterns and computed their median output similarity in the same way. A one-sided p-value was computed from the distribution of 10,000 random permutations with a Holm-Sidak correction for multiple comparisons across the groups.

\section{Rate model of the MBON-i1-FBN-7-DAN-i1 one- step feedback motif for Extended Data Fig. 11b}

To illustrate the potential effects of different FBNbaselines we modeled the isolated MBON-i1-FBN-7-DANi1 feedback motif shown in Fig. 4b with rate equations where the output of neuron type (MBON, FBN, DAN), , changed over time according to the equation $\tau_{i} \frac{d r_{i}}{d t}=$ $-r_{i}+f\left(\sum_{j} w_{i j} r_{j}+I_{i}^{\text {tonic }}\right)+I_{i}^{\text {stim }}$ where $f(x)=\frac{s}{1+e^{-k\left(x-x_{h}\right)}}$, $W$ was a matrix with positive and negative values corresponding to the direct interactions between neurons as shown in the circuit schematic of Fig. $4 \mathrm{~b}, I_{i}^{\text {tonic }}$ was a nonnegative tonic input into neuron $i, I_{i}^{\text {stim }}$ is a stimulus input provided only to MBON, $\tau_{i}$ is a time constant, and parameters $s, k$ and $x_{h}$ set the shape of the sigmoidal response. Equations were solved using ode 45 in Matlab (The Mathworks, Inc).

\section{Connectivity-constrained model of the entire} mushroom body with the feedback neurons

\section{Model dynamics}

We constructed a recurrent network model of the larval MB containing MBONs, DANs and other feedback neurons. The network receives input from $70 \mathrm{KCs}$, and external cues, such as US. The normalized firing rate $\mathbf{r}_{i}$ of neurons $i$ is modeled as a threshold-linear function of its input:

$$
\frac{d \mathbf{r}_{i}}{d t}=-\mathbf{r}_{i}(t)+f\left(\sum_{j} \mathbf{W}_{i j} \mathbf{r}_{j}(t)+\mathbf{b}^{i}+\mathbf{I}_{i}(t)\right),
$$

where $f$ represents positive rectification. Time is modeled in units of effective time constant (representing combined synaptic and membrane timescales). The connectivity matrix $\mathbf{W}_{i j}$ is constrained using the EM reconstruction. The vector $\mathbf{b}^{i}$ represents the static bias input to each neuron which determines its excitability, while $\mathbf{I}_{i}(t)$ represents time-varying external input. For MBONs, this includes external input from $\mathrm{KCs}, \mathbf{I}_{i}(t)=\sum_{k} \mathbf{W}_{i k}^{\mathrm{KC}} \mathbf{r}_{k}^{\mathrm{KC}}$. 
KCs are initially silent, but during the presentation of an odor CS, the activity of a random fraction $f$ of KCs is set to 1, leading to MBON activation. We assume all-toall KC-to-MBON connectivity. Weights $\mathbf{W}^{\mathrm{KC}}$ are initially set equal to their maximum value of $1 /\left(N_{\mathrm{KC}} f\right)$, but are modified according to a DAN-dependent synaptic plasticity rule. A weight $W(t)$ from $\mathrm{KC} k$ to an MBON in compartment $i$ evolves according to:

$$
\begin{aligned}
\frac{d w}{d t} & =-\bar{r}_{k} d_{i}+r_{k} \bar{d}_{i}, \\
\tau_{W} \frac{d W}{d t} & =w(t)-W(t),
\end{aligned}
$$

where $d_{i}$ represents the level of dopamine in the compartment (a weighted sum of DAN inputs according to the DAN-to-MBON connectivity matrix), and $r_{k}$ represents the firing rate of the $\mathrm{KC}$ (note that modifications of weights onto MBONs depend only on KC and DAN activity). The terms $\bar{r}_{k}$ and $\bar{d}_{i}$ represent the firing rate $r_{k}$ and dopamine level $d_{i}$, respectively, low-pass filtered with time constant $\tau$, which leads to an anti-Hebbian timingdependent synaptic weight update in Equation 2. The second equation results in $W(t)$ following these updates with a time constant of $\tau_{W}$ (Equation 3). For simplicity, we assume that all modulatory neurons induce plasticity according to this rule.

Weights among DANs, MBONs, and feedback neurons are constrained by the EM reconstruction. Weight matrices are initialized using synapse counts from the EM data, scaled so that the $\ell_{2}$ norm of the inputs received by each neuron $\sum_{j} \mathbf{W}_{i j}^{2}=1.5$. Only reliable connections, as defined previously, are included. Weights from neurons known to communicate using an inhibitory neurotransmitter, are then multiplied by -1 . As optimization progresses, weights from neurons of known neurotransmitter identities are constrained to maintain a consistent sign by clipping at 0 . At the beginning of a trial, MBON rates are initialized to 0 while DAN and feedback neuron rates are initialized to 0.1 . This promotes networks in which MBONs are primarily odor-driven, but some DANs and feedback neurons exhibit baseline levels of activity.

\section{Tasks}

Neuron $i$ 's external input $\mathbf{I}_{i}(t)$ represents either KC input in the case of MBONs (as described above), or US or contextual signals (depending on the task) in the case of DANs and FB neurons. We assume that $\mathbf{I}_{i}(t)=\mathbf{W}_{j}^{E} \mathbf{e}_{j}(t)$, where $\mathbf{W}^{E}$ is initialized as a random standard Gaussian variable and $\mathbf{e}_{j}(t)=0$ or 1 depending on whether signal $j$ is active. For most tasks, there are two signals (positive or negative US).

A linear readout of the MBONs determines the preference index via $P I(t)=\sum_{i \in \mathrm{MBON}} \mathbf{W}_{i}^{M} \mathbf{r}_{i}$, where $\mathbf{W}^{M}$ is initialized as a random Gaussian variable with variance $1 / N_{\mathrm{MBON}}$. Entries of $\mathbf{W}^{M}$ corresponding to MBONs whose activation is known to produce positive or negative PIs are constrained to be consistent with this sign.

Trials consist of 80 time units. In a first-order conditioning trial, a CS+ is presented for 3 time units starting randomly between $t=5$ and $t=15$, followed by a positive or negative US with a delay of 2 time units. A test $\mathrm{CS}+$ presentation occurs between $t=65$ and $t=75$, and the system must output the appropriate PI of +1 or -1 depending on the US valence during this second presentation. For extinction, an additional $\mathrm{CS}+$ presentation occurs randomly between $t=35$ and $t=45$, and the magnitude of the PI is halved for the final test CS+ presentation. For second-order conditioning, a new CS2 is presented at this time, followed by the original CS+, and the test occurs for CS2. Finally, for context-dependent conditioning, a contextual signal that determines the US valence is presented 3 time units prior to the first CS. At $t=30$ and $t=60$ firing rates are reset to their initial conditions to model an arbitrary time delay between CS presentations and preventing networks from using persistent activity, rather than synaptic plasticity, to maintain associations.

For networks trained on first-order conditioning, second-order conditioning, and extinction, training consists of random second-order conditioning and extinction trials (for which first-order conditioning is a subcomponent). On each trial, there is a $50 \%$ probability that one of the signals (e.g. the US) will be omitted, or a CS- odor will replace a CS+ odor, and the network report a PI of 0 in these cases, ensuring that only valid CS-US contingencies are learned.

\section{Optimization}

The network parameters, including all weights except for KC-to-MBON weights, as well as the biases $\mathbf{b}$, are optimized using PyTorch using the RMSprop optimizer (www.pytorch.org). Optimization consists of 1500 epochs of 30 trials each. The cost to be minimized is equal to the squared distance between the actual and target PI summed over timesteps, plus a regularization term for DAN activity. The regularization term equals $\sum_{t, i \in D A N}\left[\mathbf{r}_{i}(t)-0.1\right]_{+}^{2}$, which penalizes DAN activity that exceeds a baseline level of 0.1 . We used a timestep of $\Delta t=0.5$, although we verified that our qualitative results hold for smaller timesteps.

\section{Parameter}

$\mathrm{KC}$ coding level

Max. KC-to-MBON synaptic weight

Timing-dependent plasticity window Timescale of weight modifications

Initial MBON rate

Initial DAN rate

Initial FB neuron rate

CS/US presentation length

CS-US delay

Trial length

Timestep

RMSprop learning rate

Batch size

Number of epochs

\begin{tabular}{ll} 
Notation & Value \\
\hline$f$ & 0.1 \\
$w_{\max }$ & $1 /\left(N_{\mathrm{KC}} f\right)$ \\
$\tau$ & 5 \\
$\tau_{W}$ & 5 \\
$m_{0}$ & 0 \\
$d_{0}$ & 0.1 \\
$x_{0}$ & 0.1 \\
$T_{\text {stim }}$ & 3 \\
$\Delta T_{\mathrm{US}}$ & 2 \\
$T$ & 80 \\
$\Delta t$ & 0.5 \\
$\eta$ & 0.002 \\
$B$ & 30 \\
$N_{\text {epochs }}$ & 1500
\end{tabular}




\section{References}

1 Pavlov, I. P. \& Anrep, G. V. Conditioned reflexes; an investigation of the physiological activity of the cerebral cortex. (Oxford University Press: Humphrey Milford, 1927).

2 Bush, R. R. \& Mosteller, F. Stochastic models for learning. (Wiley, 1955).

3 Kamin, L. J. in Punishment and Aversive Behavior. (ed B. A. Campbell and R. M. Church) 279-296 (Appleton-Century-Crofts 1969).

4 Rescorla, R. A. Behavioral-Studies of Pavlovian Conditioning. Annual Review of Neuroscience 11, 329-352 (1988).

5 Quinn, W. G., Harris, W. A. \& Benzer, S. Conditioned behavior in Drosophila melanogaster. Proceedings of the National Academy of Sciences of the United States of America 71, 708-712 (1974).

6 Tempel, B. L., Bonini, N., Dawson, D. R. \& Quinn, W. G. Reward learning in normal and mutant Drosophila. Proceedings of the National Academy of Sciences of the United States of America 80, 14821486 (1983).

7 Bitterman, M. E., Menzel, R., Fietz, A. \& Schafer, S. Classical-Conditioning of Proboscis Extension in Honeybees (Apis-Mellifera). J Comp Psychol 97, 107119, doi:10.1037/0735-7036.97.2.107 (1983).

8 Davis, R. L. Traces of Drosophila memory. Neuron 70, 8-19, doi:10.1016/j.neuron.2011.03.012 (2011).

9 Dunsmoor, J. E., Niv, Y., Daw, N. \& Phelps, E. A. Rethinking Extinction. Neuron 88, 47-63, doi:10.1016/j.neuron.2015.09.028 (2015).

10 Nader, K. Reconsolidation and the Dynamic Nature of Memory. Cold Spring Harb Perspect Biol 7, a021782, doi:10.1101/cshperspect.a021782 (2015).

11 Exton-McGuinness, M. T., Lee, J. L. \& Reichelt, A. C. Updating memories-the role of prediction errors in memory reconsolidation. Behav Brain Res 278, 375384, doi:10.1016/j.bbr.2014.10.011 (2015).

12 Watabe-Uchida, M., Eshel, N. \& Uchida, N. Neural Circuitry of Reward Prediction Error. Annu Rev Neurosci, doi:10.1146/annurev-neuro-072116-031109 (2017).

13 Felsenberg, J., Barnstedt, O., Cognigni, P., Lin, S. \& Waddell, S. Re-evaluation of learned information in Drosophila. Nature 544, 240-244, doi:10.1038/nature21716 (2017).

14 Cognigni, P., Felsenberg, J. and Waddell, S. Do the right thing: neural network mechanisms of memory formation, expression and update in Drosophila. Current opinion in neurobiology 49, 5158, doi:10.1016/j.conb.2017.12.002 (2018).

15 Quinn, W. G. \& Dudai, Y. Memory phases in Drosophila. Nature 262, 576-577 (1976).
16 Dubnau, J. \& Chiang, A. S. Systems memory consolidation in Drosophila. Current opinion in neurobiology 23, 84-91, doi:10.1016/j.conb.2012.09.006 (2013).

17 Cervantes-Sandoval, I., Martin-Pena, A., Berry, J. A. \& Davis, R. L. System-like consolidation of olfactory memories in Drosophila. The Journal of neuroscience : the official journal of the Society for Neuroscience 33, 9846-9854, doi:10.1523/JNEUROSCI.0451-13.2013 (2013).

18 Lagasse, F., Devaud, J. M. \& Mery, F. A switch from cycloheximide-resistant consolidated memory to cycloheximide-sensitive reconsolidation and extinction in Drosophila. The Journal of neuroscience : the official journal of the Society for Neuroscience 29, 2225-2230, doi:10.1523/JNEUROSCI.3789-08.2009 (2009).

19 Rizley, R. C. \& Rescorla, R. A. Associations in Second-Order Conditioning and Sensory Preconditioning. J Comp Physiol Psych 81, 1-\&, doi:DOI 10.1037/h0033333 (1972).

20 Tabone, C. J. \& de Belle, J. S. Second-order conditioning in Drosophila. Learn Mem 18, 250-253, doi:10.1101/lm.2035411 (2011).

21 Schultz, W. Neuronal Reward and Decision Signals: From Theories to Data. Physiol Rev 95, 853-951, doi:10.1152/physrev.00023.2014 (2015).

22 Matsumoto, Y. \& Mizunami, M. Context-dependent olfactory learning in an insect. Learn Mem 11, 288293, doi:10.1101/lm.72504 (2004).

23 Menzel, R. Searching for the memory trace in a mini-brain, the honeybee. Learn Mem 8, 53-62, doi:10.1101/lm.38801 (2001).

24 Waddell, S. Reinforcement signalling in Drosophila; dopamine does it all after all. Current opinion in neurobiology 23, 324-329, doi:10.1016/j.conb.2013.01.005 (2013).

25 Bromberg-Martin, E. S., Matsumoto, M. \& Hikosaka, O. Dopamine in motivational control: rewarding, aversive, and alerting. Neuron 68, 815-834, doi:10.1016/j.neuron.2010.11.022 (2010).

26 Hebb, D. O. The organization of behavior; a neuropsychological theory. (Wiley, 1949).

27 Heisenberg, M. Mushroom body memoir: from maps to models. Nature reviews. Neuroscience 4, 266-275, doi:10.1038/nrn1074 (2003).

28 Rescorla, R. A. \& Wagner, A. R. in Classical Conditioning II: Current Research and Theory. 64-99 (1972).

29 Sutton, R. S. \& Barto, A. G. Reinforcement learning : an introduction. (MIT Press, 1998).

30 Montague, P. R., Dayan, P., Person, C. \& Sejnowski, T. J. Bee foraging in uncertain environments using predictive hebbian learning. Nature 377, 725-728, doi:10.1038/377725a0 (1995). 
31 Montague, P. R., Dayan, P. \& Sejnowski, T. J. A framework for mesencephalic dopamine systems based on predictive Hebbian learning. The Journal of neuroscience : the official journal of the Society for Neuroscience 16, 1936-1947 (1996).

32 Schultz, W., Dayan, P. \& Montague, P. R. A neural substrate of prediction and reward. Science 275, 1593-1599, doi:10.1126/science.275.5306.1593 (1997).

33 Doya, K. Complementary roles of basal ganglia and cerebellum in learning and motor control. Current opinion in neurobiology 10, 732-739 (2000).

34 Schultz, W. \& Dickinson, A. Neuronal Coding of Prediction Errors. Program 23, 473-500, doi:10.1146/annurev.neuro.23.1.473 (2000).

35 Waelti, P., Dickinson, A. \& Schultz, W. Dopamine responses comply with basic assumptions of formal learning theory. Nature 412, 43-48, doi:10.1038/35083500 (2001).

36 Coddington, L. T. \& Dudman, J. T. The timing of action determines reward prediction signals in identified midbrain dopamine neurons. Nature neuroscience 21, 1563-1573, doi:10.1038/s41593-018-0245-7 (2018).

37 Mirenowicz, J. \& Schultz, W. Importance of unpredictability for reward responses in primate dopamine neurons. J Neurophysiol 72, 1024-1027 (1994).

38 Cohen, J. Y., Haesler, S., Vong, L., Lowell, B. B. \& Uchida, N. Neuron-type-specific signals for reward and punishment in the ventral tegmental area. Nature 482, 85-88, doi:10.1038/nature10754 (2012).

39 Eshel, N. et al. Arithmetic and local circuitry underlying dopamine prediction errors. Nature 525, 243246, doi:10.1038/nature14855 (2015).

40 Eshel, N., Tian, J., Bukwich, M. \& Uchida, N. Dopamine neurons share common response function for reward prediction error. Nature neuroscience 19, 479-486, doi:10.1038/nn.4239 (2016).

41 Tian, J. et al. Distributed and Mixed Information in Monosynaptic Inputs to Dopamine Neurons. Neuron 91, 1374-1389, doi:10.1016/j.neuron.2016.08.018 (2016).

42 Hammer, M. An identified neuron mediates the unconditioned stimulus in associative olfactory learning in honeybees. Nature 366, 59-63 (1993).

43 Riemensperger, T., Vller, T., Stock, P., Buchner, E. \& Fiala, A. Punishment prediction by dopaminergic neurons in Drosophila. Current Biology 15, 19531960, doi:10.1016/j.cub.2005.09.042 (2005).

44 Dylla, K. V., Raiser, G., Galizia, C. G. \& Szyszka, P. Trace Conditioning in Drosophila Induces Associative Plasticity in Mushroom Body Kenyon Cells and Dopaminergic Neurons. Front Neural Circuits 11, 42, doi:10.3389/fncir.2017.00042 (2017).
45 Aso, Y. et al. The neuronal architecture of the mushroom body provides a logic for associative learning. eLife (Cambridge) 3, e04577, doi:10.7554/eLife.04577 (2014).

46 Ichinose, T. et al. Reward signal in a recurrent circuit drives appetitive long-term memory formation. eLife (Cambridge) 4, 1-18, doi:10.7554/eLife.10719 (2015).

47 Eichler, K. et al. The complete connectome of a learning and memory centre in an insect brain. Nature 548, 175-182, doi:10.1038/nature23455 (2017).

48 Takemura, S. Y. et al. A connectome of a learning and memory center in the adult Drosophila brain. eLife (Cambridge) 6, doi:10.7554/eLife.26975 (2017).

49 Zhao, X., Lenek, D., Dag, U., Dickson, B. J. \& Keleman, K. Persistent activity in a recurrent circuit underlies courtship memory in Drosophila. eLife (Cambridge) 7, doi:10.7554/eLife.31425 (2018).

50 Beier, K. T. et al. Circuit Architecture of VTA Dopamine Neurons Revealed by Systematic Input-Output Mapping. Cell 162, 622-634, doi:10.1016/j.cell.2015.07.015 (2015).

51 Edwards, N. J. et al. Circuit specificity in the inhibitory architecture of the VTA regulates cocaineinduced behavior. Nature neuroscience 20, 438-448, doi:10.1038/nn.4482 (2017).

52 Haber, S. N., Fudge, J. L. \& McFarland, N. R. Striatonigrostriatal pathways in primates form an ascending spiral from the shell to the dorsolateral striatum. The Journal of neuroscience : the official journal of the Society for Neuroscience 20, 2369-2382 (2000).

53 Felsenberg, J. et al. Integration of Parallel Opposing Memories Underlies Memory Extinction. Cell 175, 709-722.e715, doi:10.1016/j.cell.2018.08.021 (2018).

54 Pavlowsky, A., Schor, J., Placais, P. Y. \& Preat, T. A GABAergic Feedback Shapes Dopaminergic Input on the Drosophila Mushroom Body to Promote Appetitive Long-Term Memory. Current biology : CB 28, 1783-1793.e1784, doi:10.1016/j.cub.2018.04.040 (2018).

55 Cohn, R., Morantte, I. \& Ruta, V. Coordinated and Compartmentalized Neuromodulation Shapes Sensory Processing in Drosophila. Cell 163, 1742-1755, doi:10.1016/j.cell.2015.11.019 (2015).

56 Schroll, C. et al. Light-induced activation of distinct modulatory neurons triggers appetitive or aversive learning in Drosophila larvae. Current biology : CB 16, 1741-1747, doi:10.1016/j.cub.2006.07.023 (2006).

57 Rohwedder, A. et al. Four Individually Identified Paired Dopamine Neurons Signal Reward in Larval Drosophila. Current biology : CB 26, 661-669, doi:10.1016/j.cub.2016.01.012 (2016).

58 Saumweber, T. et al. Functional architecture of reward learning in mushroom body extrinsic neurons of larval Drosophila. Nature communications 9, 1104, doi:10.1038/s41467-018-03130-1 (2018). 
59 Menegas, W., Akiti, K., Amo, R., Uchida, N. \& Watabe-Uchida, M. Dopamine neurons projecting to the posterior striatum reinforce avoidance of threatening stimuli. Nature neuroscience 21, 1421-1430, doi:10.1038/s41593-018-0222-1 (2018).

60 Lammel, S., Lim, B. K. \& Malenka, R. C. Reward and aversion in a heterogeneous midbrain dopamine system. Neuropharmacology 76 Pt B, 351-359, doi:10.1016/j.neuropharm.2013.03.019 (2014).

61 Lerner, T. N. et al. Intact-Brain Analyses Reveal Distinct Information Carried by $\mathrm{SNc}$ Dopamine Subcircuits. Cell 162, 635-647, doi:10.1016/j.cell.2015.07.014 (2015).

62 Ohyama, T. et al. A multilevel multimodal circuit enhances action selection in Drosophila. Nature 520, 633-639, doi:10.1038/nature14297 (2015).

63 Heisenberg, M., Borst, A., Wagner, S. \& Byers, D. Drosophila mushroom body mutants are deficient in olfactory learning. J Neurogenet 2, 1-30 (1985).

64 Strausfeld, N. J., Sinakevitch, I., Brown, S. M. \& Farris, S. M. Ground plan of the insect mushroom body: functional and evolutionary implications. J Comp Neurol 513, 265-291, doi:10.1002/cne.21948 (2009).

65 Menzel, R. The honeybee as a model for understanding the basis of cognition. Nature reviews. Neuroscience 13, 758-768, doi:10.1038/nrn3357 (2012).

66 Owald, D. \& Waddell, S. Olfactory learning skews mushroom body output pathways to steer behavioral choice in Drosophila. Current opinion in neurobiology 35, 178-184, doi:10.1016/j.conb.2015.10.002 (2015).

67 Schurmann, F. W. Fine structure of synaptic sites and circuits in mushroom bodies of insect brains. Arthropod Struct Dev 45, 399-421, doi:10.1016/j.asd.2016.08.005 (2016).

68 Gerber, B., Stocker, R. F., Tanimura, T. \& Thum, A. S. Smelling, tasting, learning: Drosophila as a study case. Results Probl Cell Differ 47, 139-185, doi:10.1007/400_2008_9 (2009).

69 Diegelmann, S., Klagges, B., Michels, B., Schleyer, M. \& Gerber, B. Maggot learning and Synapsin function. J Exp Biol 216, 939-951, doi:10.1242/jeb.076208 (2013).

70 Widmann, A. et al. Genetic Dissection of Aversive Associative Olfactory Learning and Memory in Drosophila Larvae. PLoS genetics 12, doi:10.1371/journal.pgen.1006378 (2016).

71 Turner, G. C., Bazhenov, M. \& Laurent, G. Olfactory representations by Drosophila mushroom body neurons. J Neurophysiol 99, 734-746, doi:10.1152/jn.01283.2007 (2008).

72 Masuda-Nakagawa, L. M., Gendre, N., O'Kane, C. J. \& Stocker, R. F. Localized olfactory representation in mushroom bodies of Drosophila larvae. Proceedings of the National Academy of Sciences of the United States of America 106, 10314-10319, doi:10.1073/pnas.0900178106 (2009).
73 Campbell, R. A. et al. Imaging a population code for odor identity in the Drosophila mushroom body. The Journal of neuroscience : the official journal of the Society for Neuroscience 33, 10568-10581, doi:10.1523/JNEUROSCI.0682-12.2013 (2013).

74 Lin, A. C., Bygrave, A. M., de Calignon, A., Lee, T. \& Miesenbock, G. Sparse, decorrelated odor coding in the mushroom body enhances learned odor discrimination. Nature neuroscience 17, 559-568, doi:10.1038/nn.3660 (2014).

75 Mao, Z. \& Davis, R. L. Eight different types of dopaminergic neurons innervate the Drosophila mushroom body neuropil: anatomical and physiological heterogeneity. Frontiers in neural circuits 3, 5, doi:10.3389/neuro.04.005.2009 (2009).

76 Claridge-Chang, A. et al. Writing Memories with Light-Addressable Reinforcement Circuitry. Cell 139, 405-415, doi:10.1016/j.cell.2009.08.034 (2009).

77 Aso, Y. et al. Specific dopaminergic neurons for the formation of labile aversive memory. Current Biology 20, 1445-1451, doi:10.1016/j.cub.2010.06.048 (2010).

$78 \mathrm{Liu}, \mathrm{C}$. et al. A subset of dopamine neurons signals reward for odour memory in Drosophila. Nature 488, 512-516, doi:10.1038/nature11304 (2012).

79 Burke, C. J. et al. Layered reward signalling through octopamine and dopamine in Drosophila. Nature 492, 433-437, doi:10.1038/nature11614 (2012).

80 Perisse, E. et al. Different Kenyon Cell Populations Drive Learned Approach and Avoidance in Drosophila. Neuron 79, 945-956, doi:10.1016/j.neuron.2013.07.045 (2013).

81 Galili, D. S. et al. Converging circuits mediate temperature and shock aversive olfactory conditioning in Drosophila. Current Biology 24, 1712-1722, doi:10.1016/j.cub.2014.06.062 (2014).

82 Huetteroth, W. et al. Sweet taste and nutrient value subdivide rewarding dopaminergic neurons in Drosophila. Current biology : CB 25, 751-758, doi:10.1016/j.cub.2015.01.036 (2015).

83 Yamagata, N. et al. Distinct dopamine neurons mediate reward signals for short- and long-term memories. Proceedings of the National Academy of Sciences of the United States of America 112, 578-583, doi:10.1073/pnas.1421930112 (2015).

84 Aso, Y. \& Rubin, G. M. Dopaminergic neurons write and update memories with cell-type-specific rules. eLife (Cambridge) 5, doi:10.7554/eLife.16135 (2016).

85 Sejourne, J. et al. Mushroom body efferent neurons responsible for aversive olfactory memory retrieval in Drosophila. Nature neuroscience 14, 903910, doi:10.1038/nn.2846 (2011).

86 Pai, T.-P. et al. Drosophila ORB protein in two mushroom body output neurons is necessary for longterm memory formation. Proceedings of the National Academy of Sciences of the United States of America 110, 7898-7903, doi:10.1073/pnas.1216336110 (2013). 
87 Placais, P. Y., Trannoy, S., Friedrich, A. B., Tanimoto, H. \& Preat, T. Two pairs of mushroom body efferent neurons are required for appetitive long-term memory retrieval in Drosophila. Cell Rep 5, 769-780, doi:10.1016/j.celrep.2013.09.032 (2013).

88 Aso, Y. et al. Mushroom body output neurons encode valence and guide memory-based action selection in Drosophila. eLife (Cambridge) 3, e04580, doi:10.7554/eLife.04580 (2014).

89 Bouzaiane, E., Trannoy, S. v., Scheunemann, L., Pla??ais, P. Y. \& Preat, T. Two Independent Mushroom Body Output Circuits Retrieve the Six Discrete Components of Drosophila Aversive Memory. Cell reports 11, 1280-1292, doi:10.1016/j.celrep.2015.04.044 (2015).

90 Owald, D. et al. Activity of defined mushroom body output neurons underlies learned olfactory behavior in Drosophila. Neuron 86, 417-427, doi:10.1016/j.neuron.2015.03.025 (2015).

91 Perisse, E. et al. Aversive Learning and Appetitive Motivation Toggle Feed-Forward Inhibition in the Drosophila Mushroom Body. Neuron 90, 1086-1099, doi:10.1016/j.neuron.2016.04.034 (2016).

92 Shyu, W. H. et al. Neural circuits for longterm water-reward memory processing in thirsty Drosophila. Nature communications 8, 15230, doi:10.1038/ncomms15230 (2017).

$93 \mathrm{Wu}$, J. K. et al. Long-term memory requires sequential protein synthesis in three subsets of mushroom body output neurons in Drosophila. Sci Rep 7, 7112, doi:10.1038/s41598-017-07600-2 (2017).

94 Tanaka, N. K., Tanimoto, H. \& Ito, K. Neuronal assemblies of the Drosophila mushroom body. Journal of Comparative Neurology 508, 711-755, doi:10.1002/cne.21692 (2008).

95 Selcho, M., Pauls, D., Han, K. A., Stocker, R. F. \& Thum, A. S. The role of dopamine in Drosophila larval classical olfactory conditioning. PloS one 4, e5897, doi:10.1371/journal.pone.0005897 (2009).

96 Hige, T., Aso, Y., Rubin, G. M. \& Turner, G. C. Plasticity-driven individualization of olfactory coding in mushroom body output neurons. Nature 526, 258262, doi:10.1038/nature15396 (2015).

97 Barnstedt, O. et al. Memory-Relevant Mushroom Body Output Synapses Are Cholinergic Article Memory-Relevant Mushroom Body Output Synapses Are Cholinergic. Neuron 89, 1-11, doi:10.1016/j.neuron.2016.02.015 (2016).

98 Thum, A. S. \& Gerber, B. Connectomics and function of a memory network: the mushroom body of larval Drosophila. Current opinion in neurobiology 54, 146154, doi:10.1016/j.conb.2018.10.007 (2019).

99 Das, G., Lin, S. \& Waddell, S. Remembering Components of Food in Drosophila. Frontiers in integrative neuroscience 10, 4, doi:10.3389/fnint.2016.00004 (2016).
100 Musso, P. Y., Tchenio, P. \& Preat, T. Delayed Dopamine Signaling of Energy Level Builds Appetitive Long-Term Memory in Drosophila. Cell reports 10, 1023-1031, doi:10.1016/j.celrep.2015.01.036 (2015).

101 Luan, H., Peabody, N. C., Vinson, C. R. \& White, B. H. Refined spatial manipulation of neuronal function by combinatorial restriction of transgene expression. Neuron 52, 425-436, doi:10.1016/j.neuron.2006.08.028 (2006).

102 Pfeiffer, B. D. et al. Refinement of tools for targeted gene expression in Drosophila. Genetics 186, 735-755, doi:10.1534/genetics.110.119917 (2010).

103 Klapoetke, N. C. et al. Independent optical excitation of distinct neural populations. Nat Methods 11, 338346, doi:10.1038/nmeth.2836 (2014).

104 Aso, Y. et al. Three Dopamine pathways induce aversive odor memories with different stability. PLoS genetics 8, doi:10.1371/journal.pgen.1002768 (2012).

105 Vogt, K. et al. Shared mushroom body circuits underlie visual and olfactory memories in Drosophila. eLife (Cambridge) 3, e02395, doi:10.7554/eLife.02395 (2014).

106 Schleyer, M. et al. A behavior-based circuit model of how outcome expectations organize learned behavior in larval Drosophila. Learn Mem 18, 639-653, doi:10.1101/lm.2163411 (2011).

107 Schwaerzel, M. et al. Dopamine and octopamine differentiate between aversive and appetitive olfactory memories in Drosophila. The Journal of neuroscience : the official journal of the Society for Neuroscience 23, 10495-10502, doi:Copyright (C) 2012 by the Society for Neuroscience (2003).

108 Yamagata, N., Hiroi, M., Kondo, S., Abe, A. \& Tanimoto, H. Suppression of Dopamine Neurons Mediates Reward. PLoS biology 14, doi:ARTN e1002586, 10.1371/journal.pbio.1002586 (2016).

109 Schleyer, M., Miura, D., Tanimura, T. \& Gerber, B. Learning the specific quality of taste reinforcement in larval Drosophila. eLife (Cambridge) 4, doi:10.7554/eLife.04711 (2015).

110 Das, G. et al. Drosophila learn opposing components of a compound food stimulus. Current Biology 24, 1723-1730, doi:10.1016/j.cub.2014.05.078 (2014).

111 Kirkhart, C. \& Scott, K. Gustatory Learning and Processing in the Drosophila Mushroom Bodies. Journal of Neuroscience 35, 5950-5958, doi:10.1523/Jneurosci.3930-14.2015 (2015).

112 Kim, H., Kirkhart, C. \& Scott, K. Long-range projection neurons in the taste circuit of Drosophila. eLife (Cambridge) 6, doi:10.7554/eLife.23386 (2017).

113 Tomchik, S. M. Dopaminergic Neurons Encode a Distributed, Asymmetric Representation of Temperature in Drosophila. Journal of Neuroscience 33, 21662176a, doi:10.1523/Jneurosci.3933-12.2013 (2013). 
114 Caldwell, J. C., Miller, M. M., Wing, S., Soll, D. R. \& Eberl, D. F. Dynamic analysis of larval locomotion in Drosophila chordotonal organ mutants. Proceedings of the National Academy of Sciences of the United States of America 100, 16053-16058, doi:10.1073/pnas.2535546100 (2003).

115 Tracey, W. D., Jr., Wilson, R. I., Laurent, G. \& Benzer, S. painless, a Drosophila gene essential for nociception. Cell 113, 261-273, doi:S0092867403002721 [pii] (2003).

116 Xiang, Y. et al. Light-avoidance-mediating photoreceptors tile the Drosophila larval body wall. Nature 468, 921-926, doi:10.1038/nature09576 (2010).

$117 \mathrm{Wu}, \mathrm{Z}$. et al. A combinatorial semaphorin code instructs the initial steps of sensory circuit assembly in the Drosophila CNS. Neuron 70, 281-298, doi:10.1016/j.neuron.2011.02.050 (2011).

118 Tsubouchi, A., Caldwell, J. C. \& Tracey, W. D. Dendritic filopodia, Ripped Pocket, NOMPC, and NMDARs contribute to the sense of touch in Drosophila larvae. Current biology : CB 22, 2124-2134, doi:10.1016/j.cub.2012.09.019 (2012).

119 Ohyama, T. et al. High-throughput analysis of stimulus-evoked behaviors in Drosophila larva reveals multiple modality-specific escape strategies. PloS one 8, e71706, doi:10.1371/journal.pone.0071706 (2013).

120 Yan, Z. et al. Drosophila NOMPC is a mechanotransduction channel subunit for gentle-touch sensation. Nature 493, 221-225, doi:10.1038/nature11685 (2013).

121 Zhang, W., Yan, Z., Jan, L. Y. \& Jan, Y. N. Sound response mediated by the TRP channels NOMPC, NANCHUNG, and INACTIVE in chordotonal organs of Drosophila larvae. Proceedings of the National Academy of Sciences of the United States of America 110, 13612-13617, doi:10.1073/pnas.1312477110 (2013).

122 Robertson, J. L., Tsubouchi, A. \& Tracey, W. D. Larval defense against attack from parasitoid wasps requires nociceptive neurons. PloS one 8, e78704, doi:10.1371/journal.pone.0078704 (2013).

123 Vogelstein, J. T. et al. Discovery of brainwide neural-behavioral maps via multiscale unsupervised structure learning. Science 344, 386-392, doi:10.1126/science.1250298 (2014).

124 Jovanic, T. et al. Competitive Disinhibition Mediates Behavioral Choice and Sequences in Drosophila. Cell 167, 858-870 e819, doi:10.1016/j.cell.2016.09.009 (2016).

125 Hwang, R. Y. et al. Nociceptive neurons protect Drosophila larvae from parasitoid wasps. Current biology : CB 17, 2105-2116, doi:10.1016/j.cub.2007.11.029 (2007).

126 Eschbach, C. et al. Associative learning between odorants and mechanosensory punishment in larval Drosophila. J Exp Biol 214, 3897-3905, doi:10.1242/jeb.060533 (2011).
127 Chen, T. W. et al. Ultrasensitive fluorescent proteins for imaging neuronal activity. Nature 499, 295-300, doi:10.1038/nature12354 (2013).

128 Schneider-Mizell, C. M. et al. Quantitative neuroanatomy for connectomics in Drosophila. eLife (Cambridge) 5, doi:10.7554/eLife.12059 (2016).

129 Burgos, A. et al. Nociceptive interneurons control modular motor pathways to promote escape behavior in Drosophila. eLife (Cambridge) 7, doi:10.7554/eLife.26016 (2018).

130 Takagi, S. et al. Divergent Connectivity of Homologous Command-like Neurons Mediates SegmentSpecific Touch Respons in Drosophila. Neuron 96, 1373-1387.e1376, doi:10.1016/j.neuron.2017.10.030 (2017).

131 Liu, W. W. \& Wilson, R. I. Glutamate is an inhibitory neurotransmitter in the Drosophila olfactory system. Proceedings of the National Academy of Sciences of the United States of America 110, 1029410299, doi:10.1073/pnas.1220560110 (2013).

132 Fushiki, A. et al. A circuit mechanism for the propagation of waves of muscle contraction in Drosophila. eLife (Cambridge) 5, doi:10.7554/eLife.13253 (2016).

133 Hige, T., Aso, Y., Modi, M. N., Rubin, G. M. \& Turner, G. C. Heterosynaptic Plasticity Underlies Aversive Olfactory Learning in Drosophila. Neuron 88, 985-998, doi:10.1016/j.neuron.2015.11.003 (2015).

$134 \mathrm{Li}, \mathrm{H}$. H. et al. A GAL4 driver resource for developmental and behavioral studies on the larval CNS of Drosophila. Cell Rep 8, 897-908, doi:10.1016/j.celrep.2014.06.065 (2014).

135 Pfeiffer, B. D., Truman, J. W. \& Rubin, G. M. Using translational enhancers to increase transgene expression in Drosophila. Proceedings of the National Academy of Sciences of the United States of America 109, 6626-6631, doi:10.1073/pnas.1204520109 (2012).

136 Wilson, R. I. \& Laurent, G. Role of GABAergic inhibition in shaping odor-evoked spatiotemporal patterns in the Drosophila antennal lobe. The Journal of neuroscience : the official journal of the Society for Neuroscience 25, 9069-9079, doi:10.1523/jneurosci.2070-05.2005 (2005).

137 Daniels, R. W., Gelfand, M. V., Collins, C. A. \& DiAntonio, A. Visualizing glutamatergic cell bodies and synapses in Drosophila larval and adult CNS. J Comp Neurol 508, 131-152, doi:10.1002/cne.21670 (2008).

138 Takagawa, K. \& Salvaterra, P. Analysis of choline acetyltransferase protein in temperature sensitive mutant flies using newly generated monoclonal antibody. Neuroscience research 24, 237-243 (1996).

139 Gerber, B. et al. Timing-dependent valence reversal: A principle of reinforcement processing and its possible implications. Current Opinion in Behavioral Sciences, 114-120 (2018). 
140 Jiang, L. L.-K., A. Constructing models of heterogeneous dopamine activity. in preparation (2019).

141 Mancini, N. et al. Reversal learning in Drosophila larvae. under review (2019).

142 Brembs, B. \& Wiener, J. Context and occasion setting in Drosophila visual learning. Learn Mem 13, 618628, doi:10.1101/lm.318606 (2006).

143 Hussaini, S. A. \& Menzel, R. Mushroom body extrinsic neurons in the honeybee brain encode cues and contexts differently. The Journal of neuroscience : the official journal of the Society for Neuroscience 33, 7154-7164, doi:10.1523/jneurosci.133112.2013 (2013).

144 Shao, M., Zlatic, M. \& Cardona, A. Associative plasticity of dopaminergic neurons in Drosophila larval mushroom body. In preparation.

145 Steinberg, E. E. et al. A causal link between prediction errors, dopamine neurons and learning. Nature neuroscience 16, 966-973, doi:10.1038/nn.3413 (2013).

146 Glimcher, P. W. Understanding dopamine and reinforcement learning: the dopamine reward prediction error hypothesis. Proceedings of the National Academy of Sciences of the United States of America 108 Suppl 3, 15647-15654, doi:10.1073/pnas.1014269108 (2011).

147 Chang, C. Y. et al. Brief optogenetic inhibition of dopamine neurons mimics endogenous negative reward prediction errors. Nature neuroscience 19, 111116, doi:10.1038/nn.4191 (2016).

148 Placais, P. Y. et al. Slow oscillations in two pairs of dopaminergic neurons gate long-term memory formation in Drosophila. Nature neuroscience 15, 592-599, doi:10.1038/nn.3055 (2012).

149 Pan, W. X., Schmidt, R., Wis, J. R. \& Hyland, B. I. Tripartite mechanism of extinction suggested by dopamine neuron activity and temporal difference model. The Journal of neuroscience : the official journal of the Society for Neuroscience 28, 9619-9631, doi:10.1523/JNEUROSCI.0255-08.2008 (2008).

150 Mizunami, M. \& Matsumoto, Y. Roles of Octopamine and Dopamine Neurons for Mediating Appetitive and Aversive Signals in Pavlovian Conditioning in Crickets. Frontiers in physiology 8, 1027, doi:10.3389/fphys.2017.01027 (2017).

151 Marr, D. A theory of cerebellar cortex. J Physiol 202, 437-470 (1969).

152 Gerfen, C. R. The neostriatal mosaic: multiple levels of compartmental organization. Trends Neurosci 15, 133-139 (1992).

153 Ueoka, Y., Hiroi, M., Abe, T. \& Tabata, T. Suppression of a single pair of mushroom body output neurons in Drosophila triggers aversive associations. FEBS open bio 7, 562-576, doi:10.1002/22115463.12203 (2017).
154 Qin, H. \& Dubnau, J. Genetic disruptions of Drosophila Pavlovian learning leave extinction learning intact. Genes, brain, and behavior 9, 203-212, doi:10.1111/j.1601-183X.2009.00548.x (2010).

155 Cervantes-Sandoval, I., Phan, A., Chakraborty, M. \& Davis, R. L. Reciprocal synapses between mushroom body and dopamine neurons form a positive feedback loop required for learning. eLife (Cambridge) 6, doi:10.7554/eLife.23789 (2017).

156 Pfeiffer, B. D. et al. Tools for neuroanatomy and neurogenetics in Drosophila. Proceedings of the National Academy of Sciences of the United States of America 105, 9715-9720, doi:10.1073/pnas.0803697105 (2008).

157 Chhetri, R. K. et al. Whole-animal functional and developmental imaging with isotropic spatial resolution. Nat Methods 12, 1171-1178, doi:10.1038/nmeth.3632 (2015).

158 Jenett et al. A GAL4-Driver Line Resource for Drosophila Neurobiology. Cell Rep 2, 991-1001, doi:10.1016/j.celrep.2012.09.011 (2012).

159 Ainsley, J. A. et al. Enhanced locomotion caused by loss of the Drosophila DEG/ENaC protein Pickpocket1. Current biology : CB 13, 1557-1563 (2003).

160 Yang, C. H. et al. Control of the postmating behavioral switch in Drosophila females by internal sensory neurons. Neuron 61, 519-526, doi:10.1016/j.neuron.2008.12.021 (2009).

161 Awasaki, T., Huang, Y., O'Connor, M. B. \& Lee, T. Glia instruct developmental neuronal remodeling through TGF-beta signaling. Nature neuroscience 14, 821-823, doi:10.1038/nn.2833 (2011).

162 Suloway, C. et al. Automated molecular microscopy: the new Leginon system. Journal of structural biology 151, 41-60, doi:10.1016/j.jsb.2005.03.010 (2005).

163 Cardona, A. et al. TrakEM2 software for neural circuit reconstruction. PloS one 7, e38011, doi:10.1371/journal.pone.0038011 (2012).

164 Saalfeld, S., Fetter, R., Cardona, A. \& Tomancak, P. Elastic volume reconstruction from series of ultrathin microscopy sections. Nat Methods 9, 717-720, doi:10.1038/nmeth.2072 (2012).

165 Saalfeld, S., Cardona, A., Hartenstein, V. \& Tomancak, P. CATMAID: collaborative annotation toolkit for massive amounts of image data. Bioinformatics 25, 1984-1986, doi:10.1093/bioinformatics/btp266 (2009).

166 Berck, M. E. et al. The wiring diagram of a glomerular olfactory system. eLife (Cambridge) 5, doi:10.7554/eLife.14859 (2016).

167 Goodman, C. S., Bate, M. \& Spitzer, N. C. Embryonic development of identified neurons: origin and transformation of the $\mathrm{H}$ cell. The Journal of neuroscience : the official journal of the Society for Neuroscience 1, 94-102 (1981). 
168 Bate, M., Goodman, C. S. \& Spitzer, N. C. Embryonic development of identified neurons: segmentspecific differences in the $\mathrm{H}$ cell homologues. The Journal of neuroscience : the official journal of the Society for Neuroscience 1, 103-106 (1981).

169 Costa, M., Manton, J. D., Ostrovsky, A. D., Prohaska, S. \& Jefferis, G. S. NBLAST: Rapid, Sensitive Comparison of Neuronal Structure and Construction of Neuron Family Databases. Neuron 91, 293-311, doi:10.1016/j.neuron.2016.06.012 (2016).

170 Schindelin, J. et al. Fiji: an open-source platform for biological-image analysis. Nat Methods 9, 676-682, doi:10.1038/nmeth.2019 (2012).

171 Nern, A., Pfeiffer, B. D. \& Rubin, G. M. Optimized tools for multicolor stochastic labeling reveal diverse stereotyped cell arrangements in the fly visual system. Proceedings of the National Academy of Sciences of the United States of America 112, E29672976, doi:10.1073/pnas.1506763112 (2015).

172 Selcho, M., Pauls, D., Huser, A., Stocker, R. F. \& Thum, A. S. Characterization of the octopaminergic and tyraminergic neurons in the central brain of Drosophila larvae. J Comp Neurol 522, 3485-3500, doi:10.1002/cne.23616 (2014).

173 Pauls, D., Selcho, M., Gendre, N., Stocker, R. F. \& Thum, A. S. Drosophila larvae establish appetitive olfactory memories via mushroom body neurons of embryonic origin. The Journal of neuroscience : the official journal of the Society for Neuroscience 30, 1065510666, doi:10.1523/jneurosci.1281-10.2010 (2010).

174 Marley, R. \& Baines, R. A. Dissection of third-instar Drosophila larvae for electrophysiological recording from neurons. Cold Spring Harb Protoc 2011, doi:10.1101/pdb.prot065656 (2011). 
EscbibRxiv, prepkint idei: hlttp3:/doi.org/10.1101/649731; this version posted May 27, 2019. The copyright holder for this preprint (which was not certified by peer review) is the author/funder. All rights reserved. No reuse allowed without permission.

\section{Extended Data Figure 1}

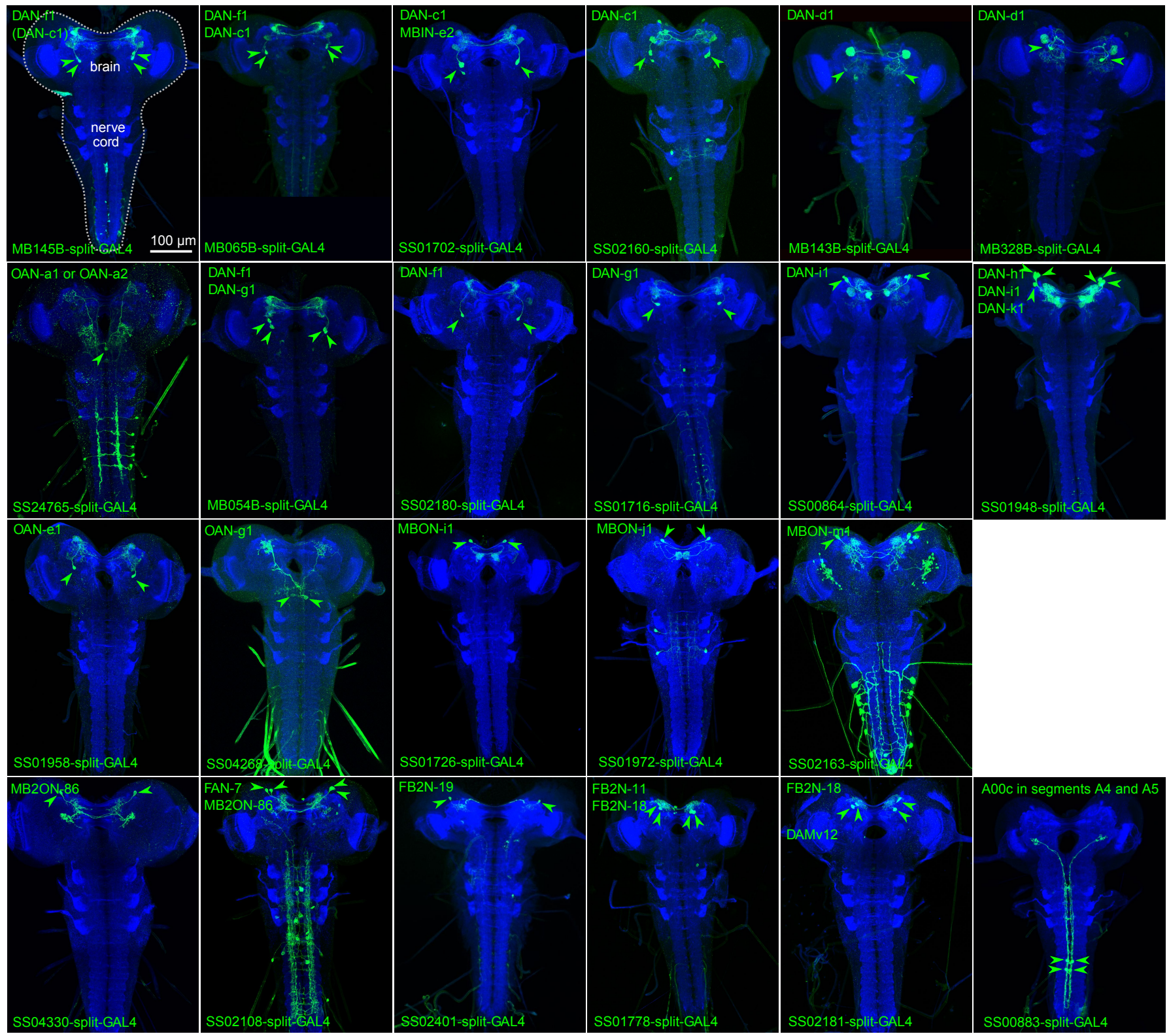

Extended Data Figure 1: Expression patterns of Split-GAL4 lines.

Each panel shows a confocal maximum intensity projection of the complete CNS of third-instar larvae (indicated by the dotted line in the first pannel), with the neuropil labeled with anti-N-Cad antibody (blue) and the Split-GAL4 line expression pattern revealed by driving UAS-myr-GFP (green). Arrowheads indicate cell bodies of identified neurons. 
EscbibRxily, prephint idei: hittpsoy/doi.org/10.1101/649731; this version posted May 27, 2019. The copyright holder for this preprint (which was not certified by peer review) is the author/funder. All rights reserved. No reuse allowed without permission.

\section{Extended Data Figure 2}

a
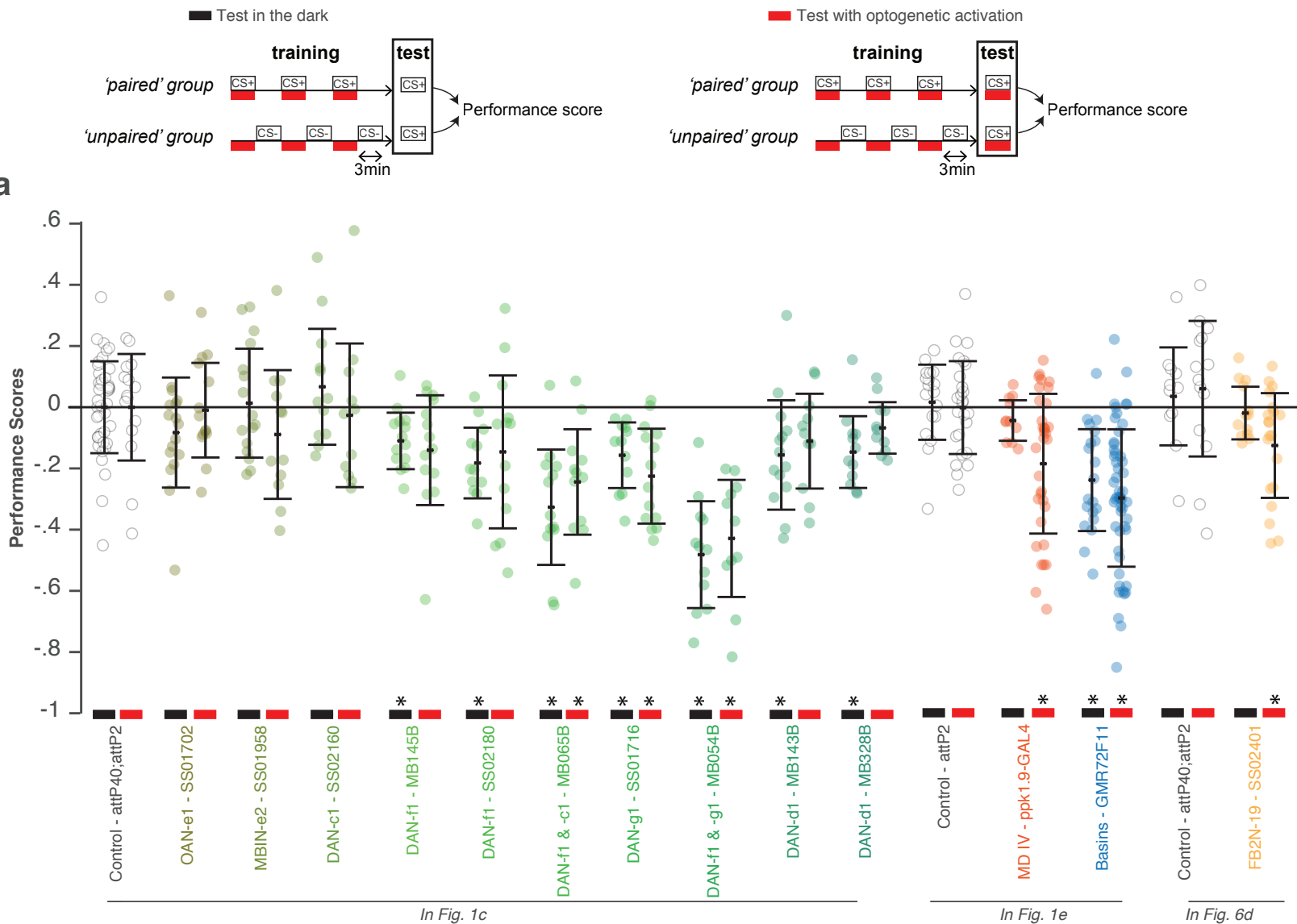

b ounpaired group

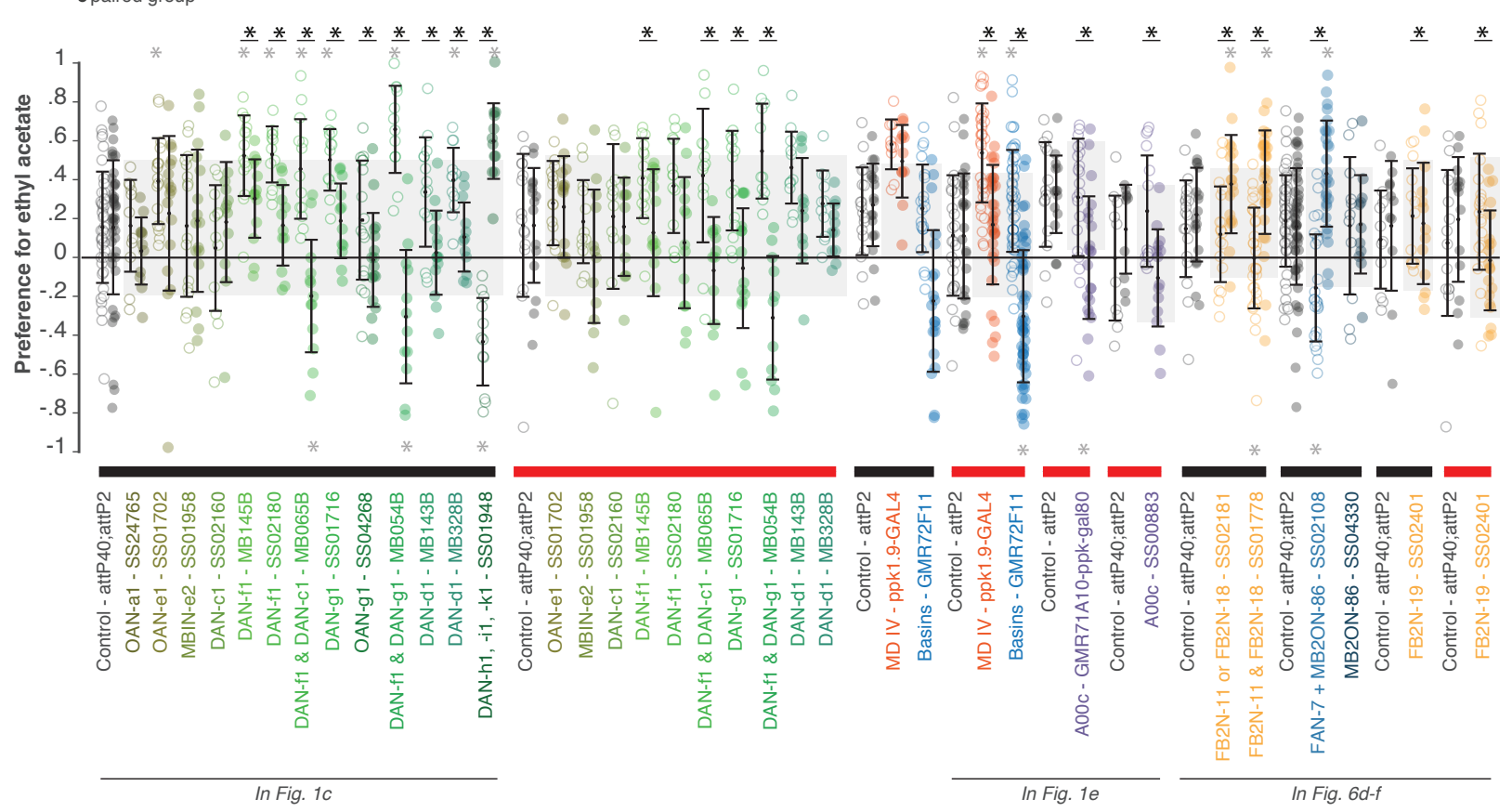

Extended Data Figure 2:

Detailed characterization of associative memories formed through different kinds of "optogenetic punishments" or "optogenetic rewards" 
Extended Data Figure 2: Continues from prior page.

a With some natural punishments, aversive memory is behaviorally expressed by trained Drosophila larvae only if the punishment is present at the moment of the test (Hendel and Gerber, 2006; Eschbach et al. 2011; Schleyer et al. 2011). Here we assayed olfactory aversive memories in two ways: both with or without optogenetic punishment (red and black bars, respectively) during the retention test. We found that, aversive memory formed by DAN activation (green) was expressed to the same extent with or without the DANs activated during the retention test. Similarly memories evoked by Basins activation (blue) can be expressed without activation of Basins during test. However memory evoked by the activation of nociceptive MD IV neurons (orange) or FB2N-19 (yellow) was fully expressed only if these neurons were active again during the retention test. Mean and standard deviations are shown, *: p-value from a Mann-Whitney test comparison to the scores of the corresponding control group (open circles) compared to the value 0.05 adjusted with a Holm-Bonferroni correction for multiple comparisons.

b Preference scores for the trained odor, ethyl acetate, when it was paired (paired group, closed circles) or not paired (unpaired group, open circles) with optogenetic punishments or rewards. Odor preference was decreased and increased, respectively, relative to genetic controls, after pairing the odor with the presence and absence of the following optogenetic punishments: coactivation of the aversive DAN-f1 and DAN-g1, co-activation of DAN-f1 and DAN-c1, or activation of Basins. On the contrary, odor preference was increased and decreased, respectively, relative to genetic controls, after pairing the odor with the presence and absence of the following optogenetic rewards: the co-activation of DAN-h1, -i1, and -k1 (dark green); the activation of FB2N-18 and FB2N-11 (yellow), or activation of FAN-7 (blue-gray). Thus, both absence of odor in the unpaired group of animals, as well as the presence of odor in the paired group of animals can be associated with the activation of some DANs or some of their afferent neurons. For other DANs or afferent neurons, only paired (e.g. A00c, purple)) or only unpaired (e.g. the modulatory DAN-f1, the nociceptive MD IV sensory neuron, or FB2N-19) contingency significantly affected odor preference with respect to the control group. Ether of these two observed types of effects can contribute to the negative or positive learning performance indexes plotted in Fig. 1c, 1e, 5f', 5g' and 5h'. Black *: p-value $<0.05$ from a Wilcoxon test comparison between paired and unpaired group. Grey *: p-value $<0.05$ from a Mann-Whitney U test comparison between the preference scores for a given group (paired or unpaired) and the preference scores (for paired or unpaired protocol, respectively) obtained by the control line shown on the left of each set of data. Sample sizes: $N=\mathbf{4 2}, 11,17,16,12,14,12,13,12,16,12,14,12,12,15,14,12,11$, $14,13,12,14,11,11,11, \mathbf{1 8}, 11,20, \mathbf{2 5}, 33,52, \mathbf{1 4}, 21, \mathbf{1 4}, 14, \mathbf{1 8}, 18,31, \mathbf{5 2}, 27,11, \mathbf{1 1}, 13,10,20$ (control groups in bold). 


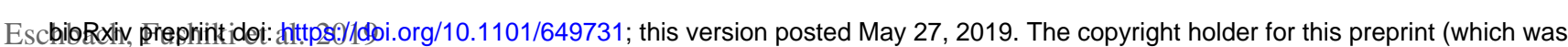
not certified by peer review) is the author/funder. All rights reserved. No reuse allowed without permission.

\section{Extended Data Figure 3}

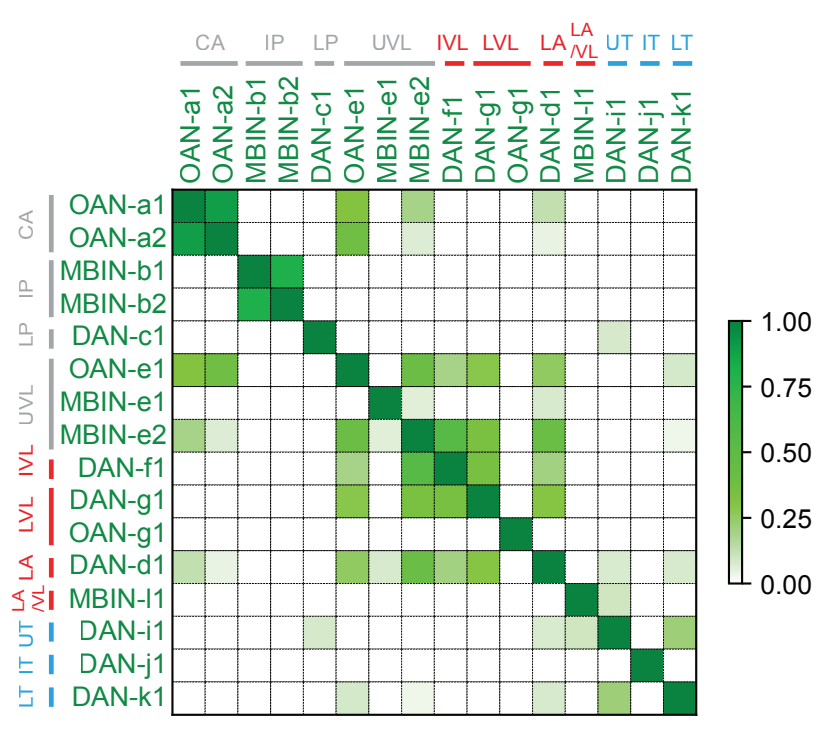

Extended Data Figure 3: Matrix of similarity between modulator neurons based on the amount of common input.

Similarity is obtained by counting the total number of inputs onto a row modulator neuron that are also inputs of the column modulator neurons, and divide by the total number of inputs onto the column modulator neurons. An input here is a connection, consisting typically of many synapses, from a specific cell type onto the modulator neuron. Inputs onto a modulator neuron type are considered if the pair of left and right neurons presynaptic to the pair of left and right modulator neurons is each above a threshold of $1 \%$ (e.g. the presynaptic neuron makes 3 synapses onto a neuron with 300 postsynaptic sites) and the sum of both is over $3.3 \%$ (e.g. the sum of both connections is above 10 synapses for receiving neurons with 300 postsynatic sites). 
EscbibRxiv, prepkint idei: hlttp3:/doi.org/10.1101/649731; this version posted May 27, 2019. The copyright holder for this preprint (which was not certified by peer review) is the author/funder. All rights reserved. No reuse allowed without permission.

\section{Extended Data Figure 4}

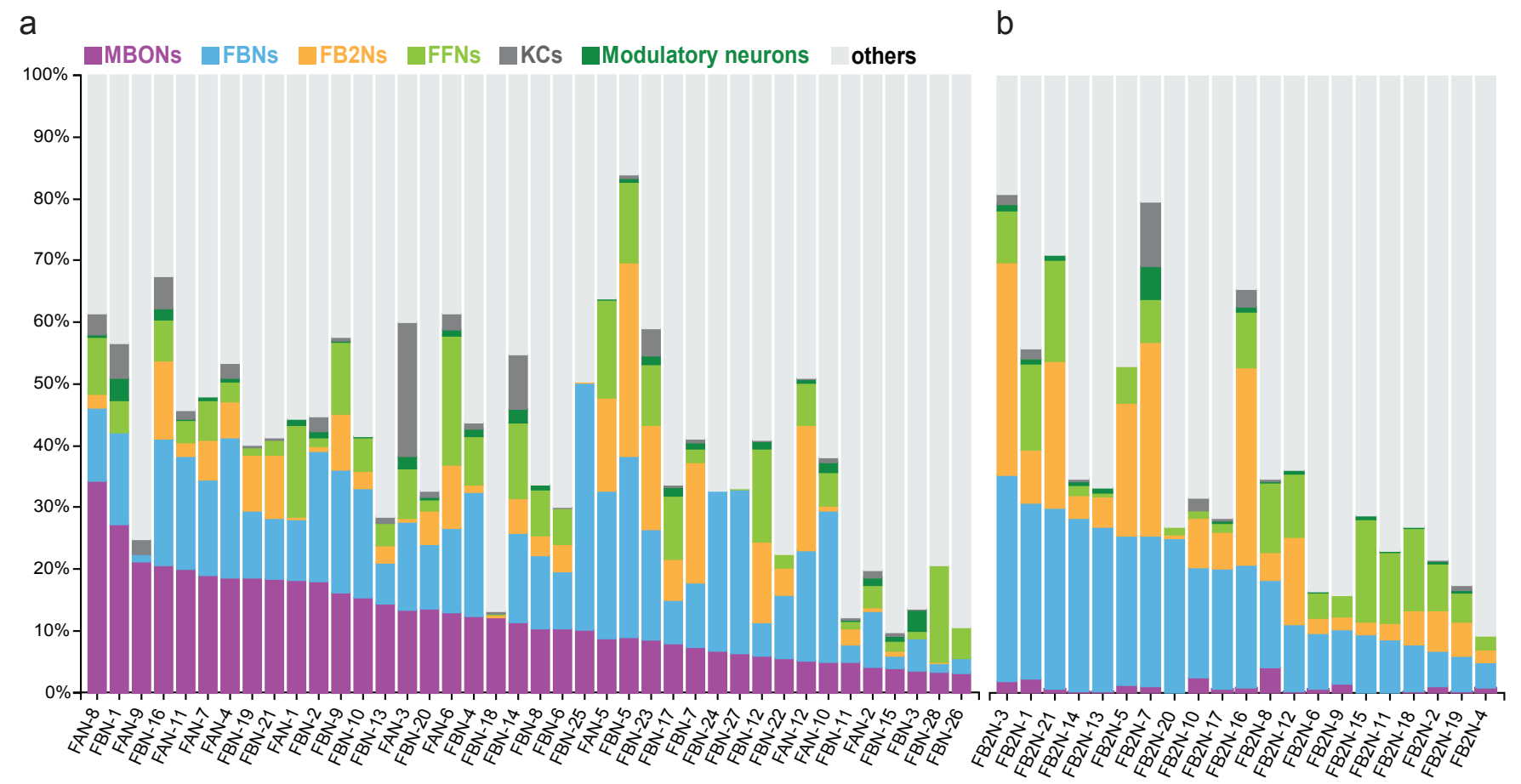

C
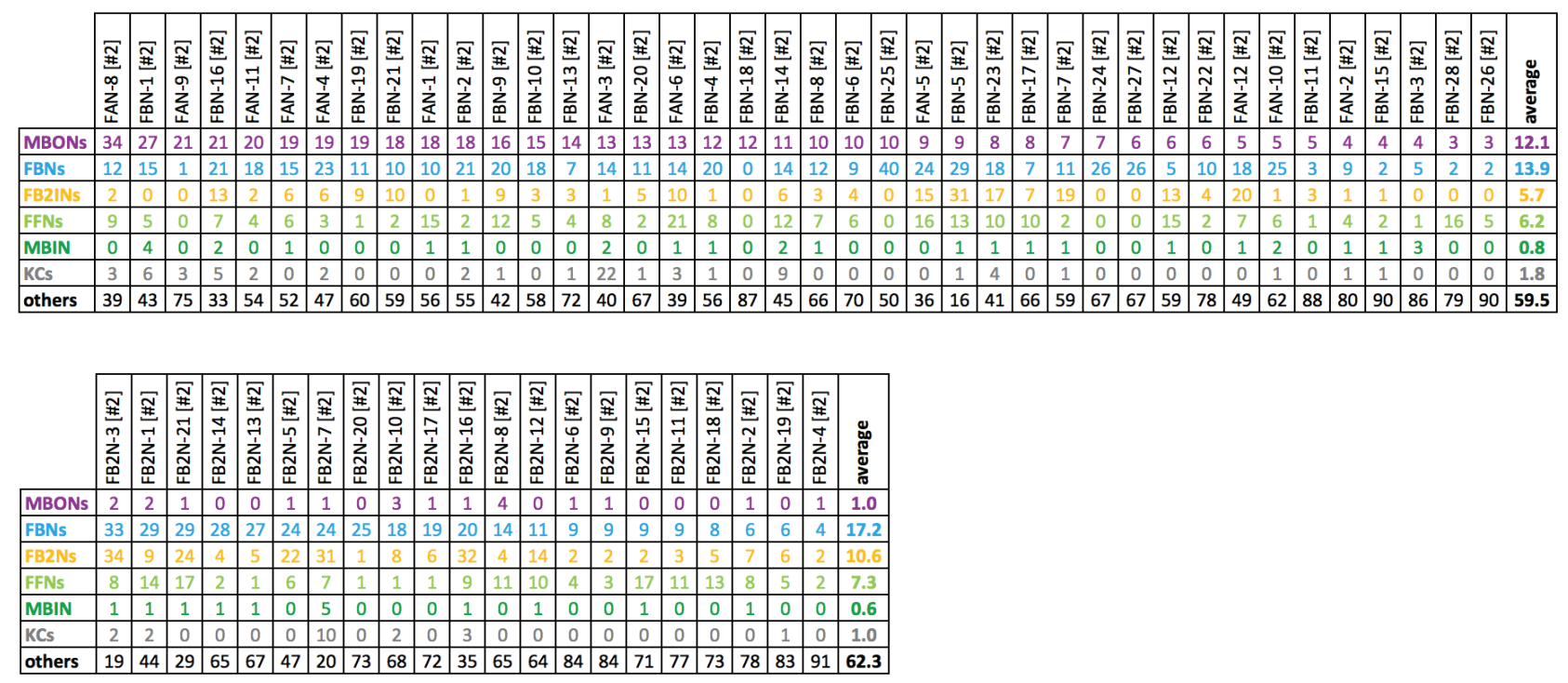

Extended Data Figure 4: Input onto feedback neurons. Figure shows the fractions of total dendritic input each pre-modulatory neuron (FBN, FB2N or FFN) receives from KCs, modulatory neurons, MBONs, FBNs, FB2Ns, FFNs, and from other non-MB neurons (others). a FBNs receive on average $12 \%$ of their inputs directly from MBONs and most of them also receive inputs from other FBNs, with an average of $26 \%$ from MBONs and other FBNs combined (see also Extended Data Fig. 13a). b FB2Ns receive inputs both from FBNs (on average 17\%) and from other FB2Ns (on average 28\% from FBNs and FB2Ns combined). Many feedback neurons also receive a significant fraction of input from other unknown neurons from other brain areas (other than MB), suggesting that the feedback about the learnt valences of stimuli is integrated with or modulated by other information. c Tables show percent of inputs onto FBNs (top) and FB2Ns (bottom) from MBONs, FBNs, FB2Ns, FFNs, modulatory neurons, and Kenyon cells. 


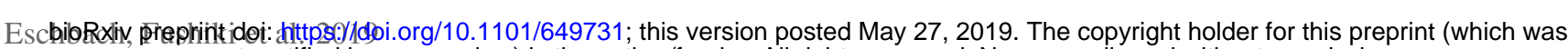
not certified by peer review) is the author/funder. All rights reserved. No reuse allowed without permission.

\section{Extended Data Figure 5}

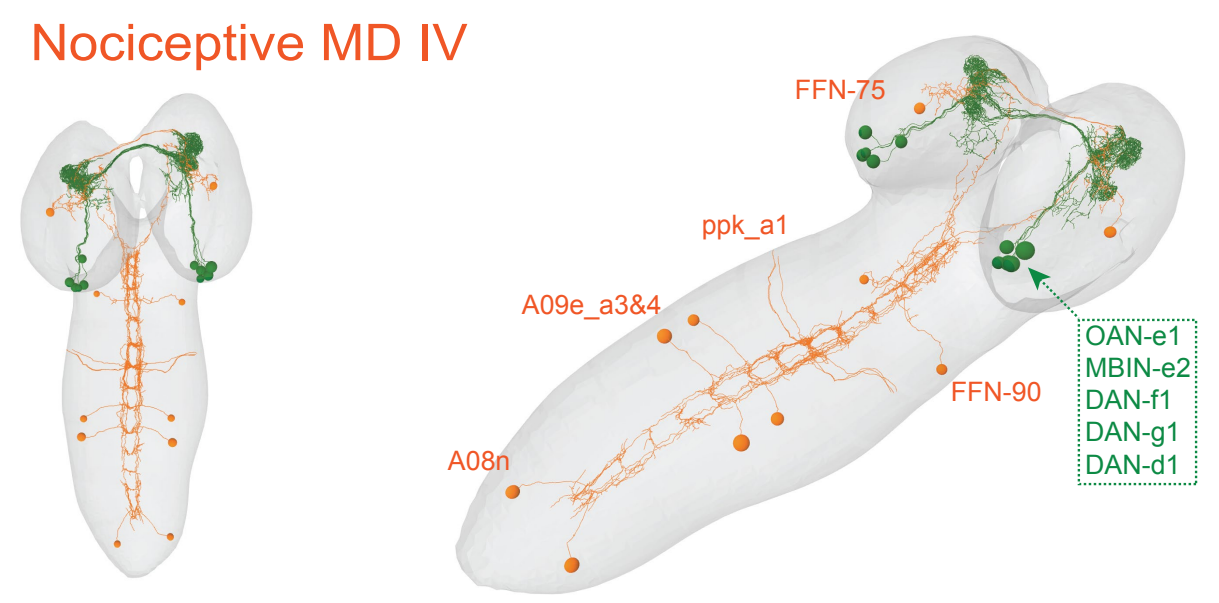

\section{Basin multisensory}
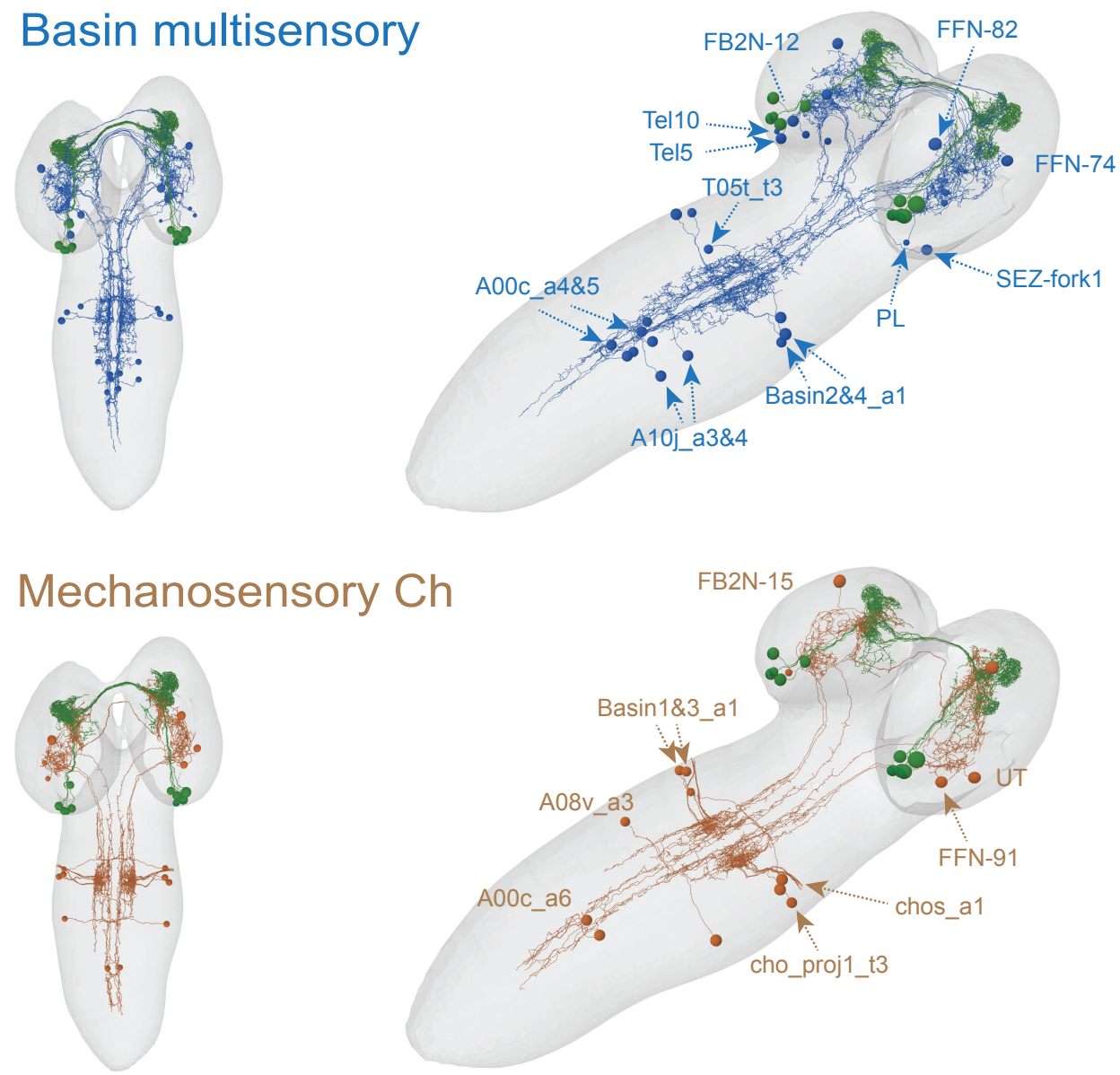

Extended Data Figure 5: (related to Fig. 2f) EM reconstruction of feedforward pathways from sensory neurons to the VL modulatory neurons.

Figure shows reconstructed neurons in the nociceptive (orange), multisensory Basin (blue) and mechanosensory (brown) pathways projecting to the VL modulatory neurons (green). 
EscbiloRXiv, prepkintidei: hittps://doi.org/10.1101/649731; this version posted May 27, 2019. The copyright holder for this preprint (which was not certified by peer review) is the author/funder. All rights reserved. No reuse allowed without permission.

\section{Extended Data Figure 6}

a

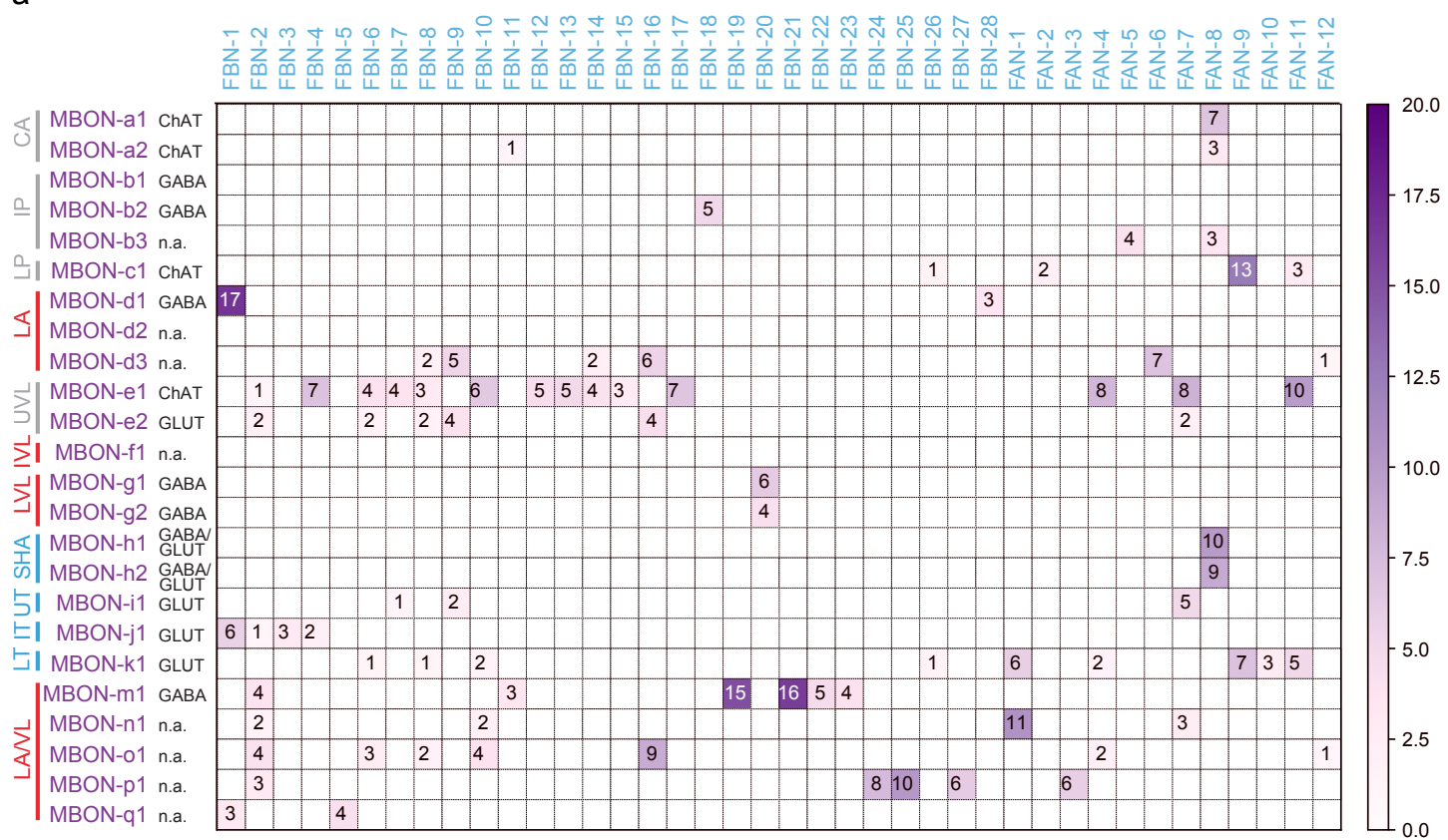

b

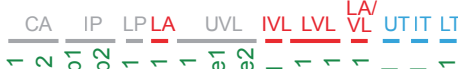

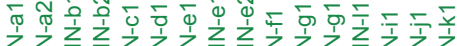

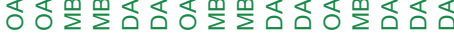

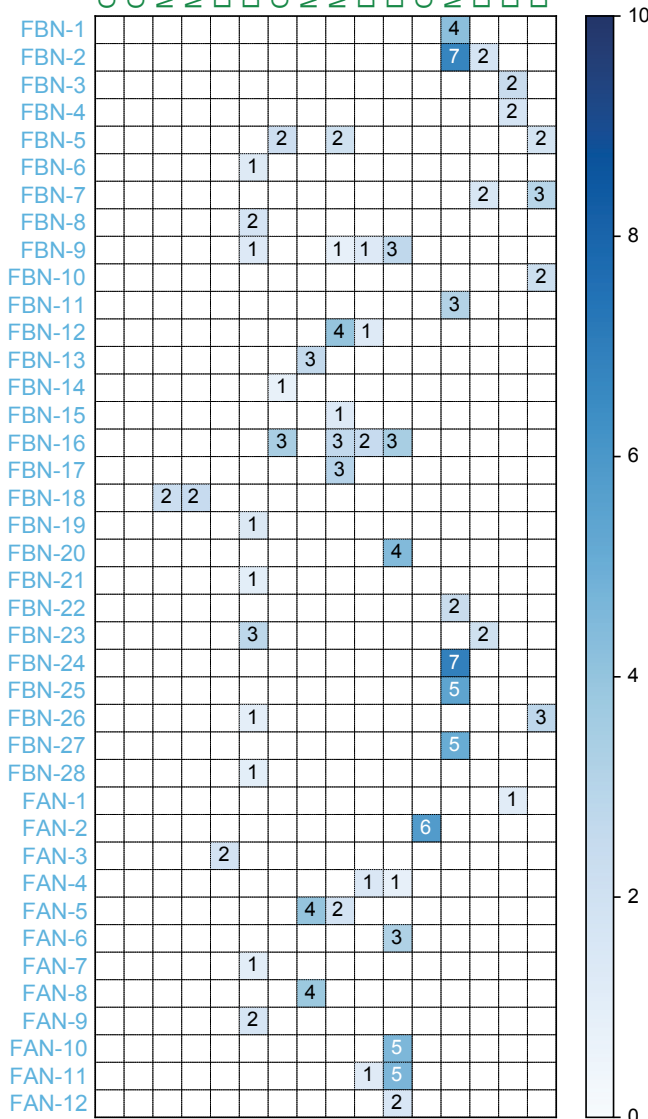

Extended Data Figure 6: (related to Fig. 3) Connectivity matrices between MBONs, FBNs, and modulatory neurons. 
EscbibRxiv prepkintidei: Alttps://doi.org/10.1101/649731; this version posted May 27, 2019. The copyright holder for this preprint (which was not certified by peer review) is the author/funder. All rights reserved. No reuse allowed without permission.

Extended Data Figure 6: Continues from prior page.

Connectivity matrix showing normalized synaptic input (expressed as \% input, computed as in Fig. 2b) each postsynaptic neuron (columns) receives from each presynaptic neuron (rows).

Only reliable connections are shown for which both the left and right homologous connections have at least 3 synapses, and their sum is at least 10 , and for which the postsynaptic neuron receives at least $1 \%$ of input from the presynaptic neuron.

MBONs and modulatory neurons are grouped by MB lobe. CA, Calyx; IP, Intermediate peduncle; LP, Lower peduncle; LA, Lateral appendix; UVL, Upper vertical lobe; IVL, Intermediate vertical lobe; LVL, Lower vertical lobe; SHA, Shaft; UT, Upper toe; IT, Intermediate toe; LT, Lower toe. a Each row and column represents MBONs (presynaptic neurons) and FBNs (postsynaptic neurons), respectively. b Each row and column represents FBNs (presynaptic neurons) and modulatory neurons (postsynaptic neurons), respectively. 
EscbiloRxiv, prephintidei: hittpsog/doi.org/10.1101/649731; this version posted May 27, 2019. The copyright holder for this preprint (which was not certified by peer review) is the author/funder. All rights reserved. No reuse allowed without permission.

\section{Extended Data Figure 7}

a

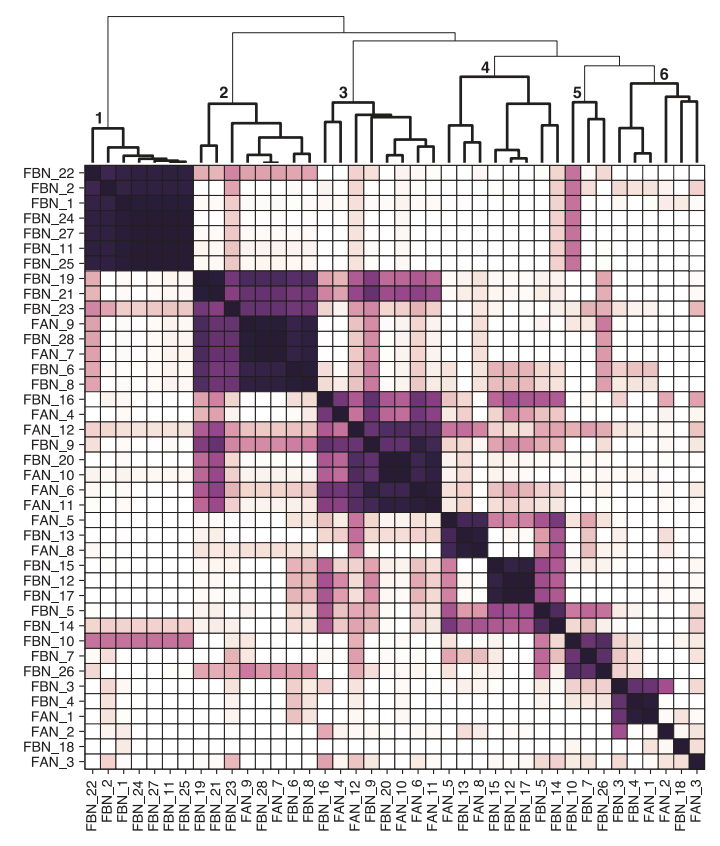

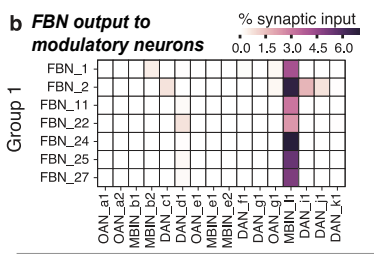
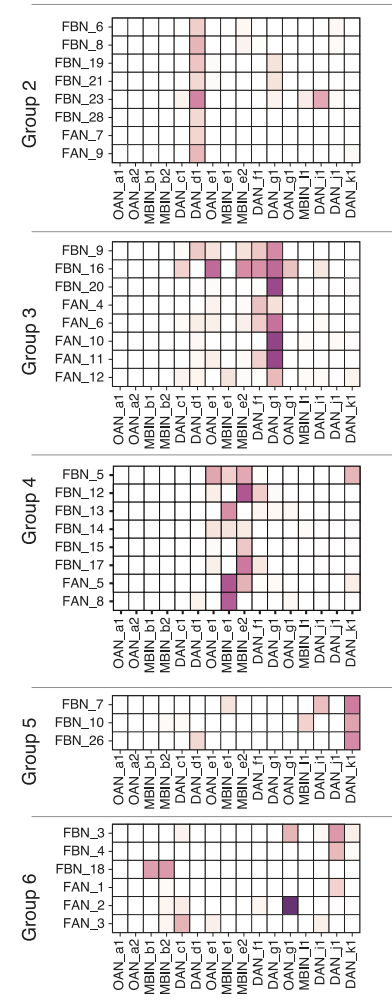

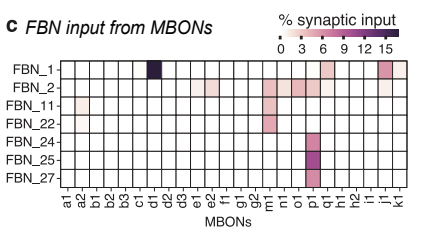

d
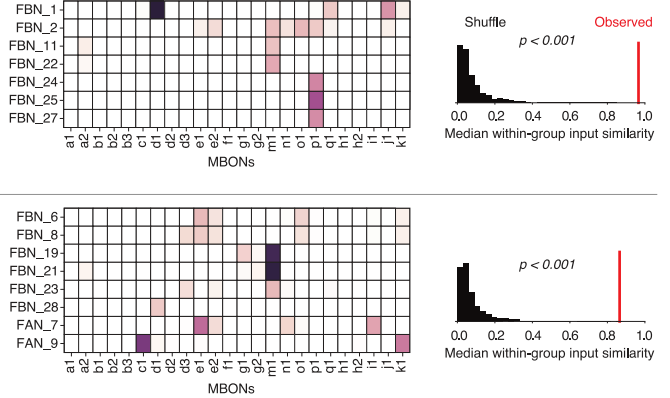

MBONs
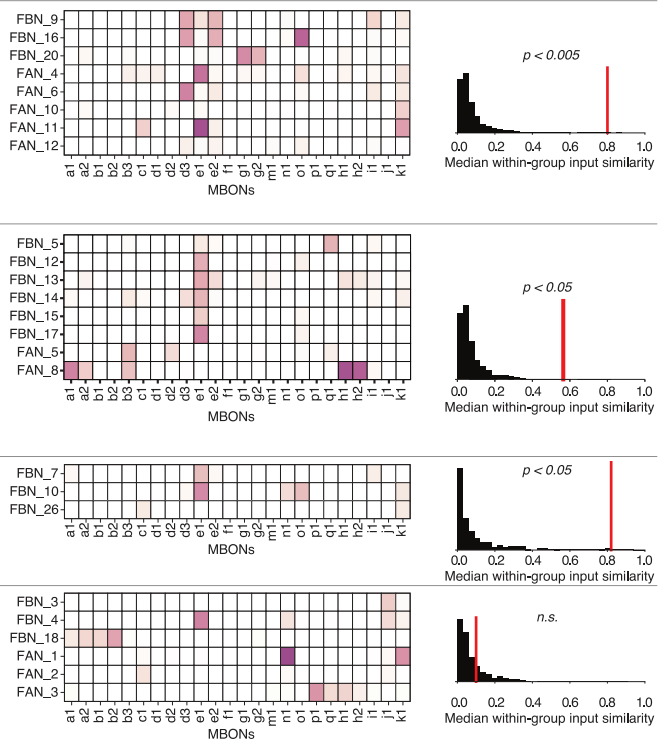

Extended Data Figure 7: Clustering FBNs based on output onto modulatory neurons.

a Heat map of FBN similarity based on the pattern of FBN synaptic output across all modulatory neurons. The similarity between a pair of FBNs was computed as the cosine similarity between the vectors of normalized synaptic output onto all modulatory neurons. Indices were ordered by agglomerative clustering with average linkage (dendrogram shown at top). We highlight six groups of FBNs defined by similarities in their output patterns (bold lines in dendrogram, numbered).

b Heat maps showing patterns of synaptic output from FBNs to modulatory neurons for output groups highlighted in a. Each group corresponds to several FBNs strongly targeting one or a small number of modulatory neurons, suggesting that some modulatory neurons are more strongly modulated than others.

c Heat maps showing patterns of input onto FBNs from MBONs for the output groups highlighted in a.

d The observed similarity in the input patterns between FBNs within each group, compared to shuffled data. For each group (as defined by output patterns), we computed the observed median of cosine similarity of the input vectors across all pairs of neurons (red line). In Groups 1-5, the neurons clustered by outputs had more input output patterns than would be expected by chance. To determine significance, we compared the observed similarity to the distribution of the median cosine similarity for randomly permuted samples from the observed population of input vectors (black histograms, $n=10000$ randomized trials). A Holm-Sidak correction was applied to $\mathrm{p}$-values to correct for multiple comparisons. 


\section{Extended Data Figure 8}

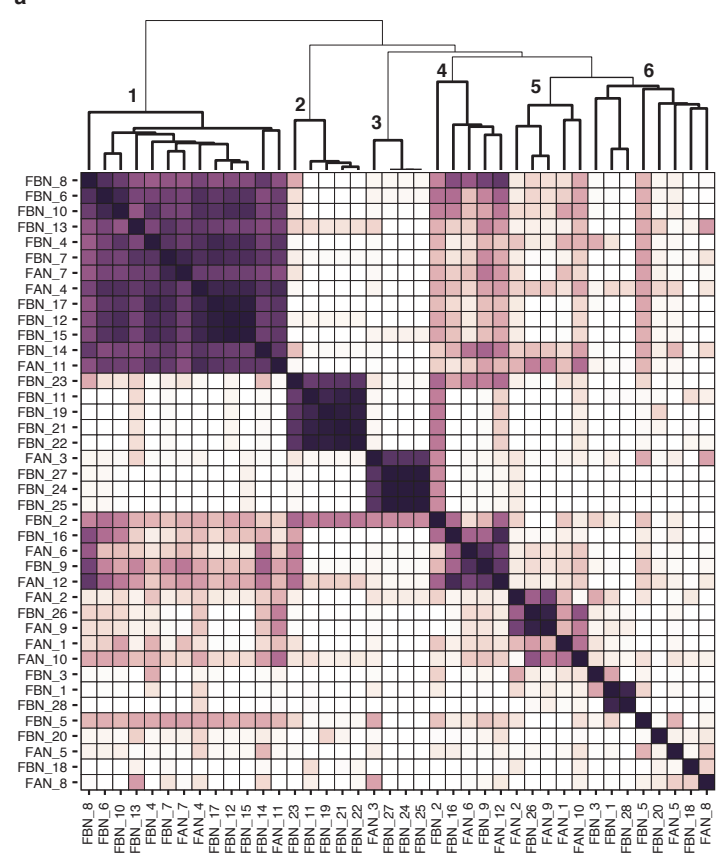

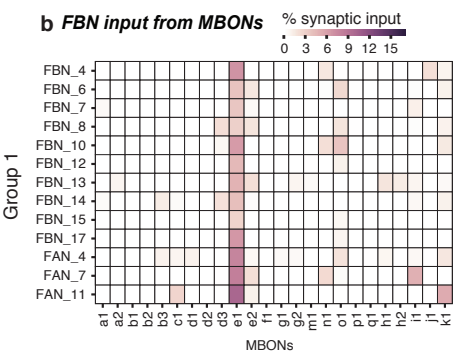

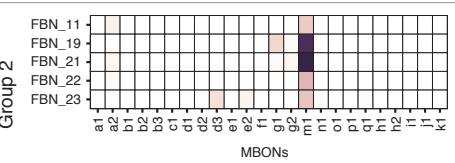

MBONs
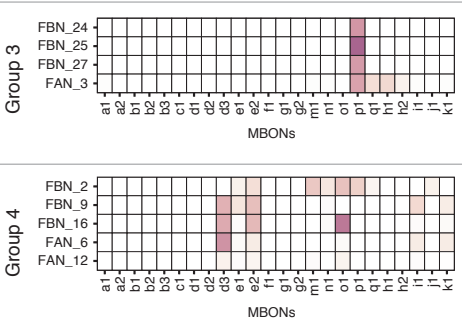

MBONs

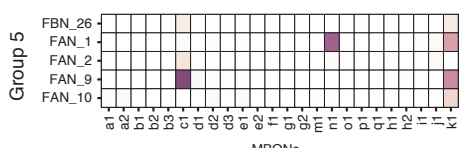

MBONs

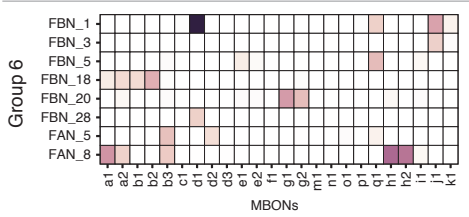

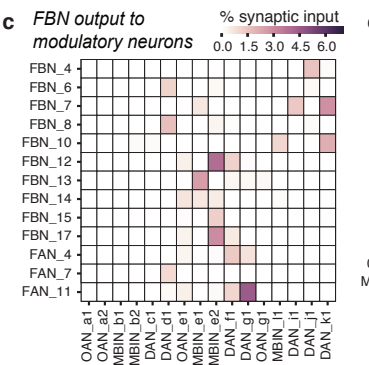

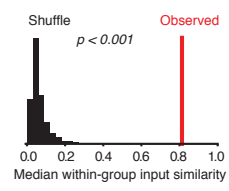

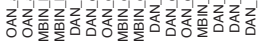

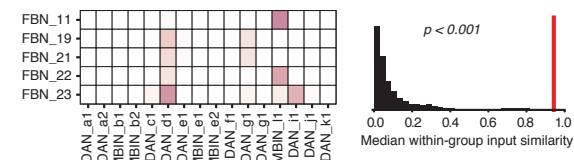

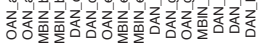
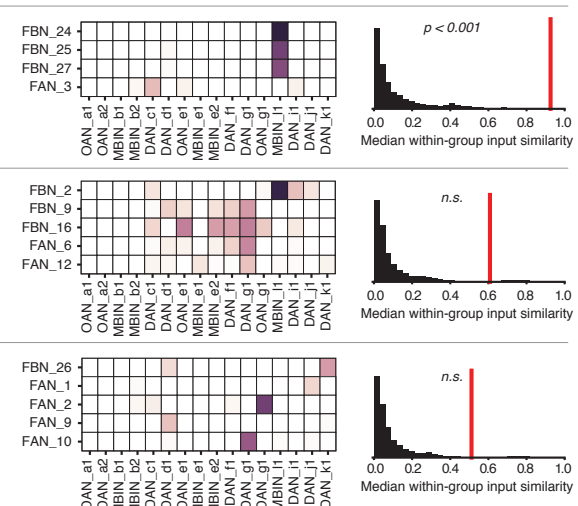

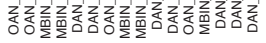
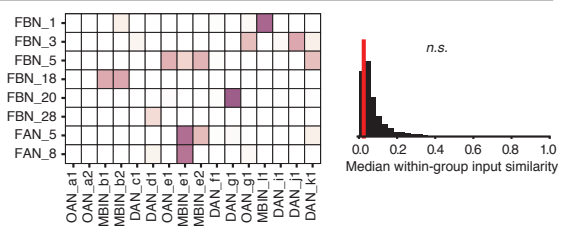

Extended Data Figure 8: Clustering FBNs based on input from MBONs.

a Heat map of FBN similarity based on the pattern of FBN synaptic inputs from MBONs. The similarity between a pair of FBNs was computed as the cosine similarity between the vectors of their normalized synaptic inputs from all MBONs. Indices were ordered by agglomerative clustering with average linkage (dendrogram shown at top). We highlight six groups of FBNs defined by similarities in their input patterns (bold lines in dendrogram, numbered).

b Heat maps showing patterns of input from MBONs onto FBNs for the input groups highlighted in a. In all cases, connectivity is measured in normalized synaptic input on the postsynaptic neuron. Most input groups receive dominant input from a single specific MBON (Groups 1,2,5) or small group of MBONs (Groups 3 and 4), while Group 6 is not well-clustered and contains a variety of dissimilar input patterns.

c Heat maps showing the patterns of synaptic output from FBNs to modulatory neurons for the input groups highlighted in a. d The observed similarity in the output patterns between FBNs within each group, compared to shuffled data. For each group clustered by input pattern, we computed the observed median of cosine similarity of the output vectors across all pairs of neurons (red line). In Groups 1,2, and 3, the neurons clustered by inputs had more similar output patterns than would be expected by chance. To determine significance, we compared the observed similarity to the distribution of the median cosine similarity for randomly permuted samples from the observed population of output vectors (black histograms, $n=10000$ randomized trials). A Holm-Sidak correction was applied to p-values to correct for multiple comparisons. 
EscbibRxiv, prepkint idei: Alttp3:/doi.org/10.1101/649731; this version posted May 27, 2019. The copyright holder for this preprint (which was not certified by peer review) is the author/funder. All rights reserved. No reuse allowed without permission.

\section{Extended Data Figure 9}

a

Direct projections of MBONs onto modulatory neurons

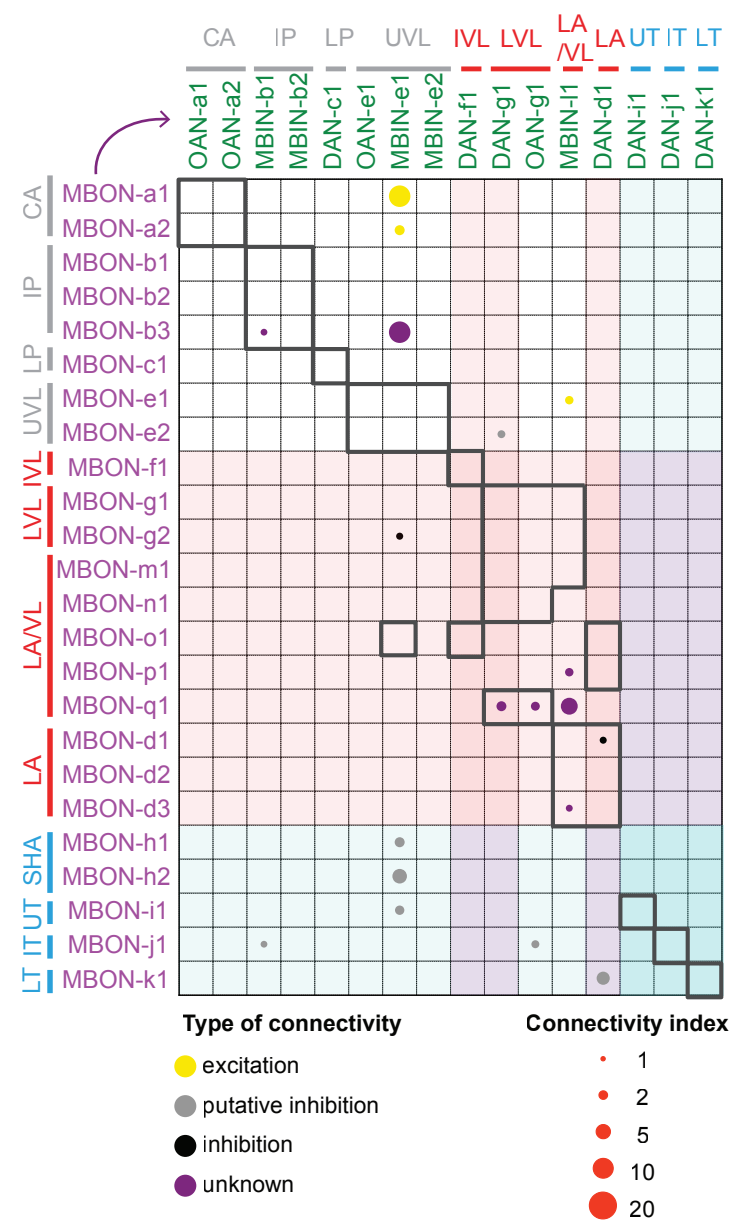

b

Indirect projections of MBONs onto modulatory neurons

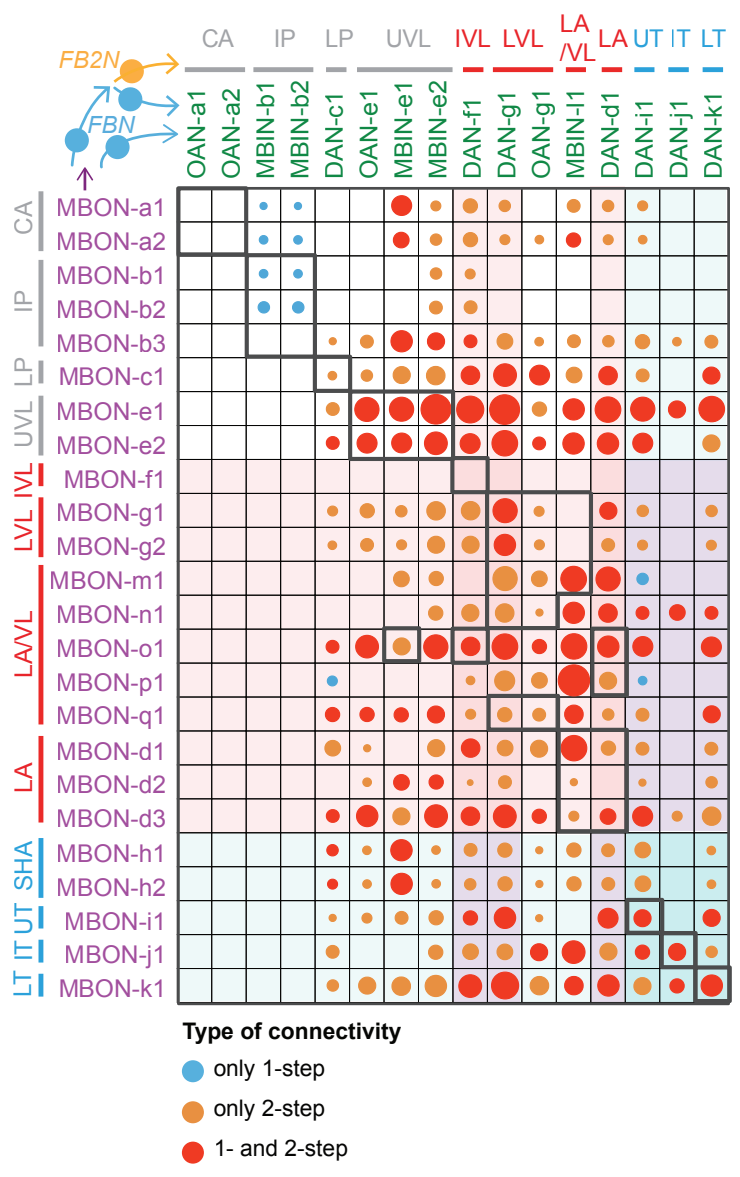

Extended Data Figure 9: Direct MBONs to modulatory neuron connectivity is very sparse, in contrast to the very dense connectivity via one-and two-step feedback pathways.

a Connectivity matrix showing normalized synaptic input (expressed as \% input, computed as in $b$ ) each modulatory neuron (columns) receives from each MBON (rows). Only reliable connections for which the postsynaptic neuron receives at least $1 \%$ of input from the presynaptic neuron are shown.

When the neurotransmitter of the MBON is known, the circle is color-coded to represent type of connection: excitatory (ChAT) or probably disinhibitory (GluT). Color shades represent the valence of the memory formed in a given compartment (red: aversive memory, blue: appetitive memory). True within-compartment feedback connections from an MBON that receives direct synaptic input from that modulatory neuron are boxed in bold.

Very few modulatory neurons receive direct input from MBONs, in contrast to the dense connectivity between MBONs and modulatory neurons via the indirect one- and two-step feedback pathways (b).

b Connectivity matrix showing indirect connections between MBONs and modulatory neurons via one-step and/or two-step feedback pathways. The matrix was obtained by summing the matrices from Fig. $3 b$ and Fig. $5 e$. The color indicates the type of indirect connection existing between a given MBON and a given DAN. Bubble size represents a connectivity index computed as in Fig. $3 b$ and Fig. 5e. A connectivity index of 1 or 10 means that for all connections comprising that indirect feedback pathway the presynaptic neuron accounts for $1 \%$ and $10 \%$ of input onto that postsynaptic neuron, respectively.

One- and two-step feedback drastically increases the connectivity between MBONs and modulatory neurons, compared to direct connections $(a)$. 


\section{Extended Data Figure 10}

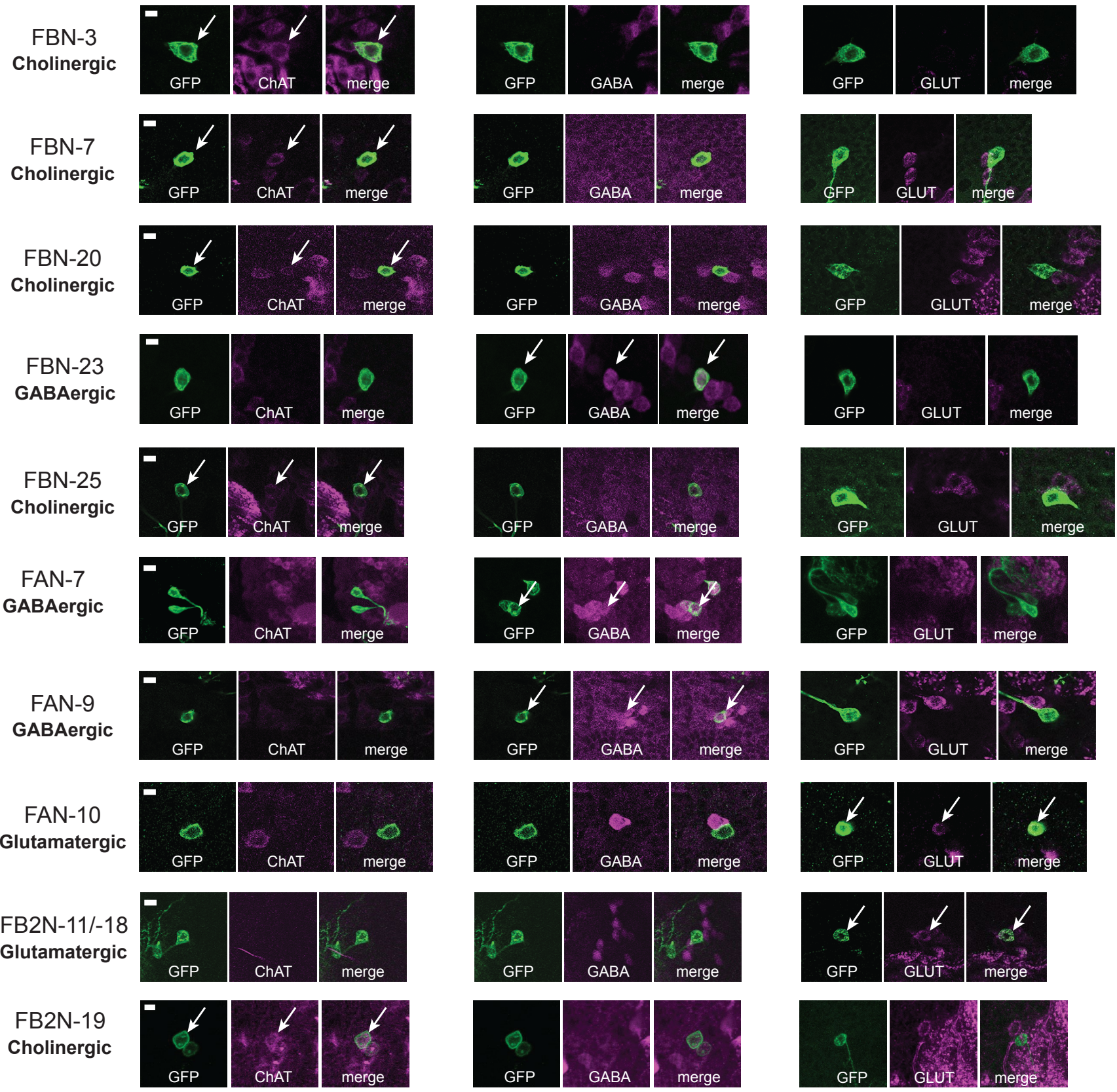

Extended Data Figure 10: Identification of neurotransmitters expressed in some FBNs/FB2Ns. Neurotransmitter expression detected in neuron somata using antibody labelling. We identified GAL4 lines that drive gene expression in some of the FBN or FB2N neurons and used them to express GFP in these neurons. We stained central nervous systems with antibodies against GFP and either ChAT (choline acetyltransferase), GABA (gamma aminobutyric acid) or GLUT (vesicular glutamate transporter). Each row shows from left to right: the name of the individual neuron, anti-GFP (green), anti-ChAT (magenta), and both antibody stainings combined; anti-GFP (green), anti-GABA (magenta), and both antibody stainings combined; anti-GFP (green) and antiGLUT (magenta), and both antibody stainings combined. Whether a cell is cholinergic, GABAergic or glutamatergic is listed at the beginning of each row under the neuron name. Images show confocal maximum intensity projections of specific neuronal cell bodies. Scale bars: $5 \mu \mathrm{m}$. 


\section{Extended Data Figure 11}

MBON-i1 > CsChrimson DAN-i1:
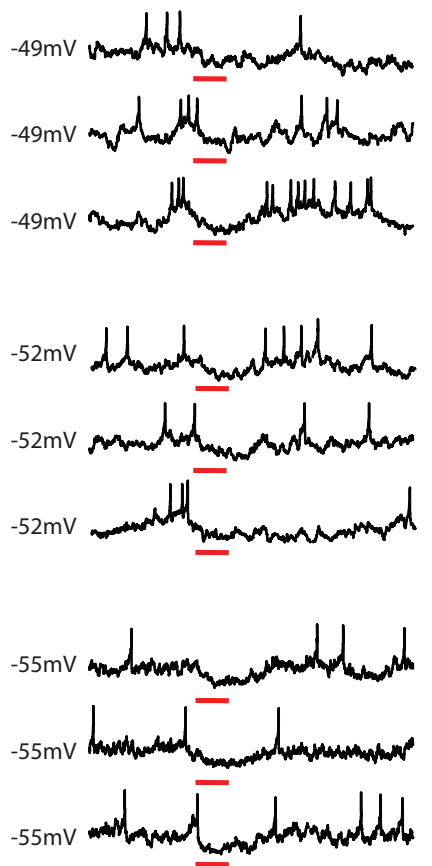

b

Putative gating mechanism for inhibition:

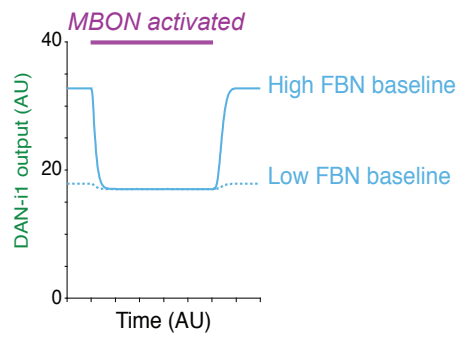

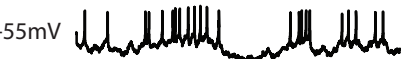
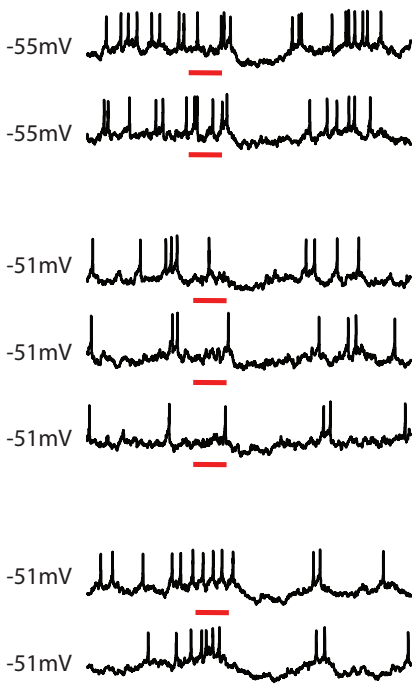

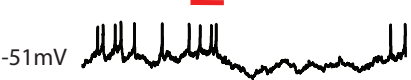

C

Putative pathway for inhibition at the offset:

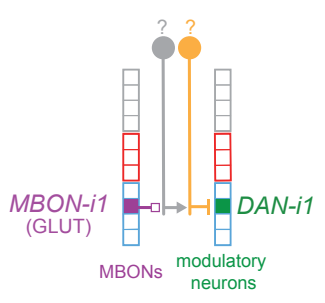

MBON-m1 > CsChrimson DAN-11:
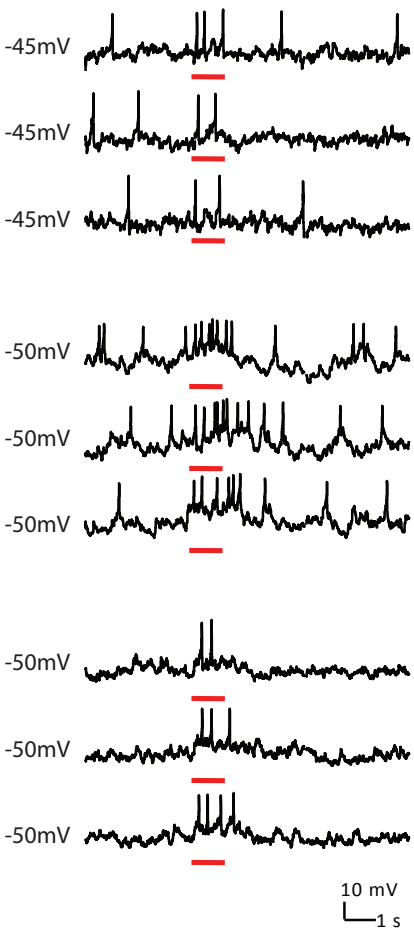

Extended Data Figure 11: (related to Fig. 4) Electrophysiological recording of DAN-i1 during optogenetic stimulation of MBON-i1 or MBON-m1.

a Raw electrophysiological traces of whole-cell patch-clamp recording of DAN-i1 (medial lobe) during optogenetic activation of the medial lobe MBON-i1 of the same compartment. Left: 3/9 animals had a long-latency inhibitory responses in DAN-i1 at the onset of MBON-i1 activation $(55.3 \pm 17.3 \mathrm{~ms}, \mathrm{n}=60$ traces from 3 animals $)$. For each of them, three individual traces are shown. Inhibition at the onset in some animals but not others might result from distinct baseline states of FBN, as modeled in $\mathbf{b}$. Right: 4/9 animals had even longer latency inhibitory responses in DAN-i1 at the offset of MBON-i1 activation (95.3 $\pm 43.5 \mathrm{ms,}$ $\mathrm{n}=80$ trials from 4 animals). Traces from 3 example animals are shown. Inhibition at the offset might be result of post-inhibitory rebound within a two-step feedback pathway like the one shown in c. b Simple rate model of the circuit comprising the MBONi1, FBN-7 and DAN-i1, as shown in Fig. $4 b$ illustrating how distinct FBN-7 baseline states could result in an inhibitory response, or no inhibitory response to the onset of MBON-1 activation in DAN-i1. Neuronal activity was modeled as a leaky integrator with logistic function response. The high and low baseline states are modeled as a stronger or weaker tonic excitatory input into the FBN-7. Purple bar indicates MBON activation. In the model, in the high-FBN-7 and low-FBN-7 baseline states, onset of MBON-1 activation evokes an inhibitory response and no response in DAN-i1, respectively. c Schematic diagram showing a putative two-step feedback pathway between MBON-i1 and the DAN-i1 that could mediate inhibition at the offset of MBON-i1 activity. This proposed mechanism would involve post-inhibitory rebound in the neuron postsynaptic to MBON-i1. An example two-step feedback pathway that could mediate this response comprises FBN-9 and FB2N-3, but the neurotransmitters of these neurons have not yet been identified due to the lack of appropriate GAL4 lines. d Raw electrophysiological traces of whole-cell patch-clamp recording of DAN-i1 (medial lobe) during optogenetic activation of the vertical lobe MBON-m1. Three out of three animals had a long-latency excitatory response in DAN-i1 to optogenetic activation of MBON-m1 $(51.3 \pm 7.7 \mathrm{~ms}, \mathrm{n}=45 \mathrm{trials}$ from 3 animals). For each of them, three individual traces are shown. Red rectangles indicate time of optogenetic stimulation. Numbers indicate absolute baseline potential. 


\section{Extended Data Figure 12}
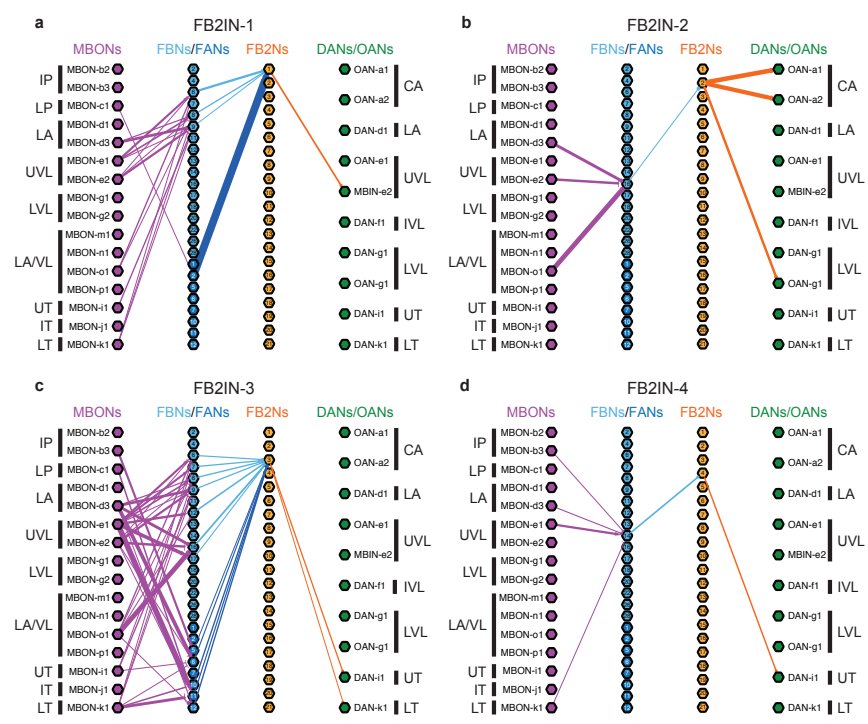

d FB2IN-4
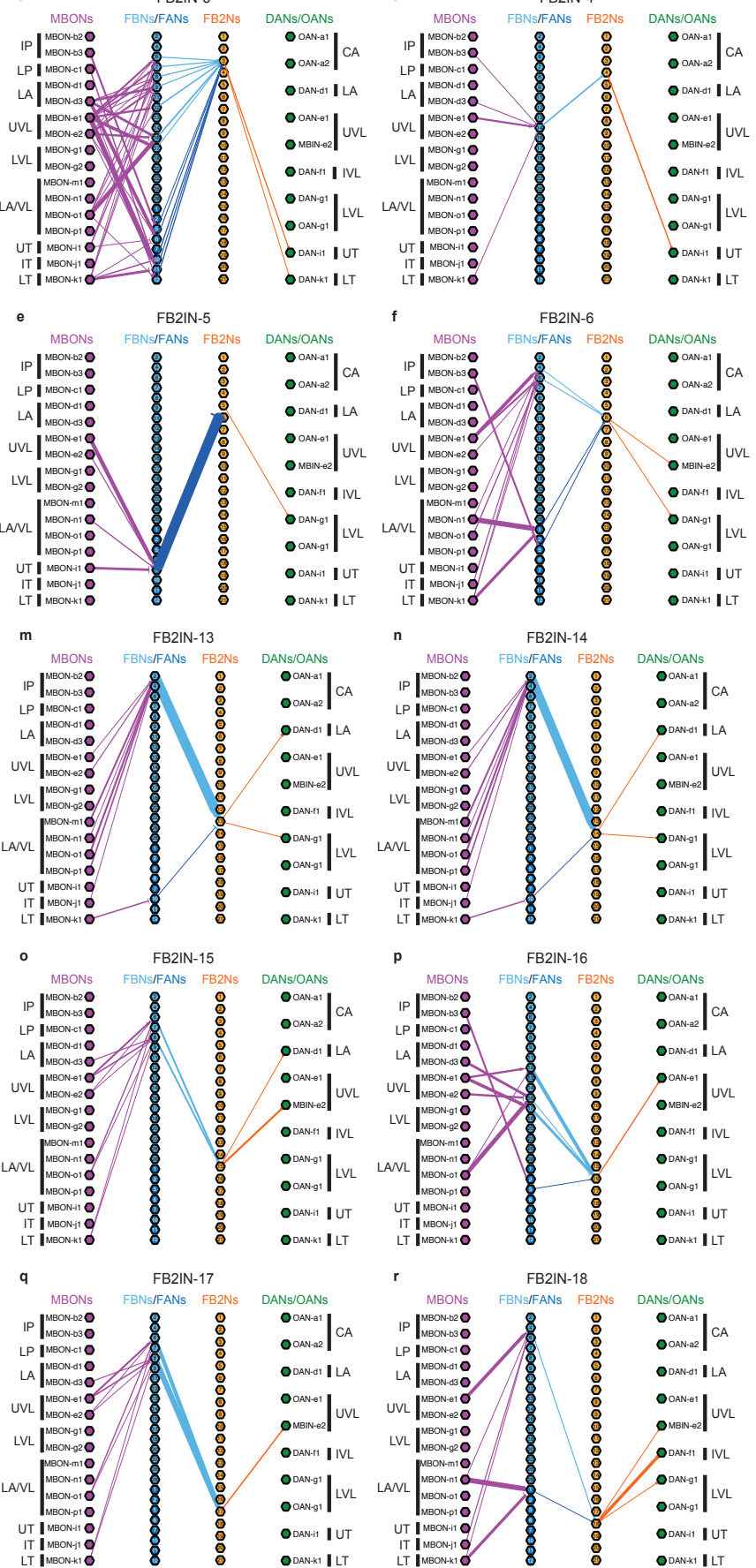
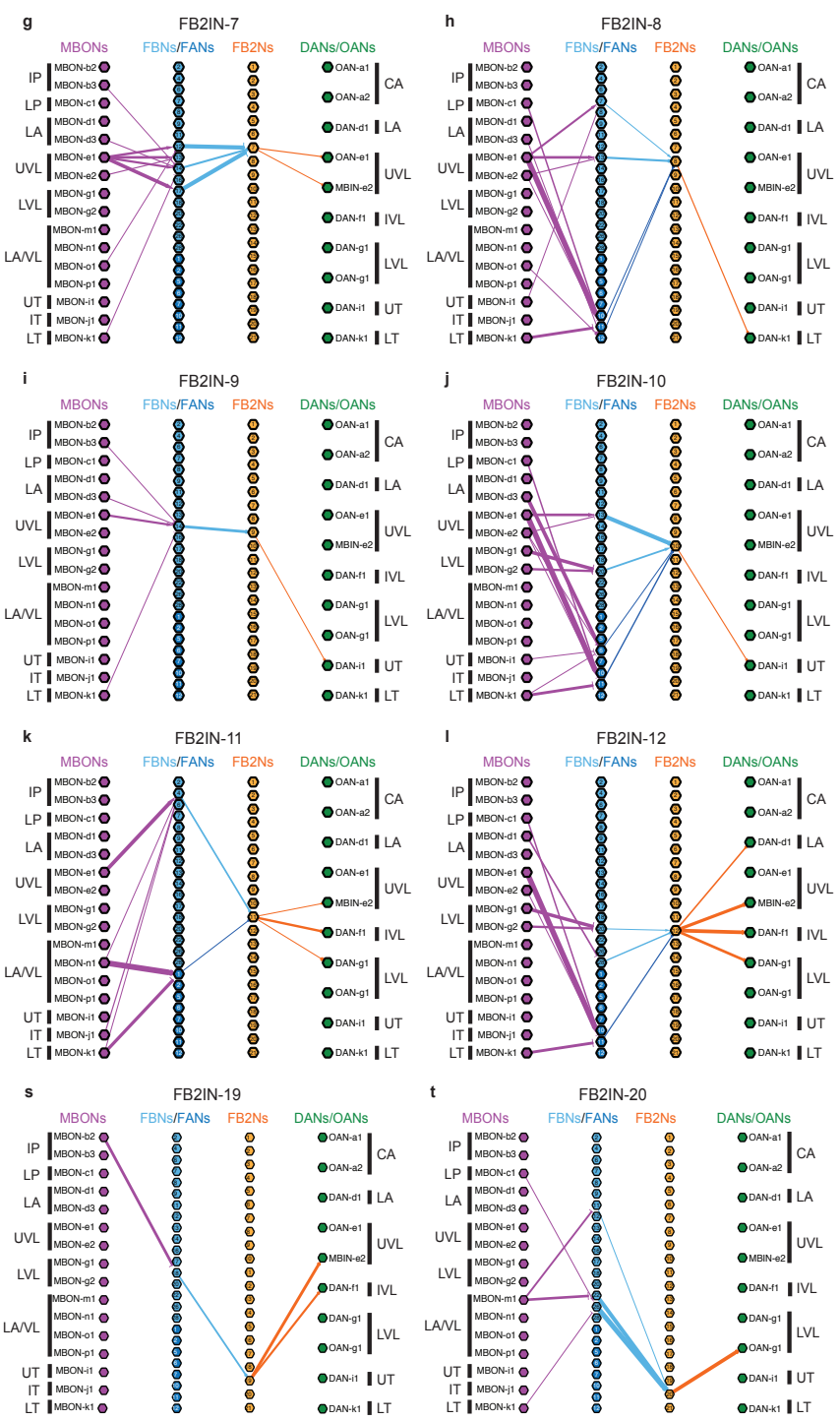

FB2IN-19
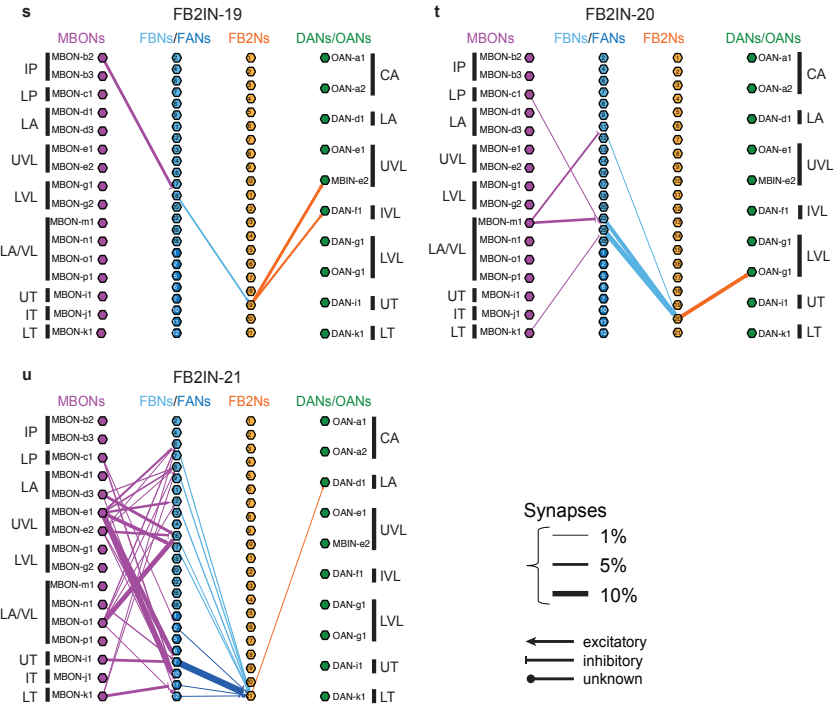

Extended Data Figure 12: (related to Fig. 5) All identified within- and cross-compartment two-step feedback pathways, via FB2Ns. $21 \mathrm{FB} 2 \mathrm{~N}$ pairs receive indirect input from MBONs and direct input from FBNs and synapsed onto modulatory neurons. Some FB2Ns synapsed onto modulatory neurons in their own compartment (true second-order feedback), as well as in other compartments, while other FB2Ns synapsed onto modulatory neurons in other compartments (pure second-order feedacross pathways). Thickness of the arrows is proportional to normalized synaptic input (as in (Fig. 2e-f). Arrowhead, line, square, and circle denote excitatory (ChAT), inhibitory (GABA), probably inhibitory (GLUT), and unknown neurotransmitter identity, respectively. 
EscbibRXiv, prephint idei: alttp8:/doi.org/10.1101/649731; this version posted May 27, 2019. The copyright holder for this preprint (which was not certified by peer review) is the author/funder. All rights reserved. No reuse allowed without permission.

\section{Extended Data Figure 13}

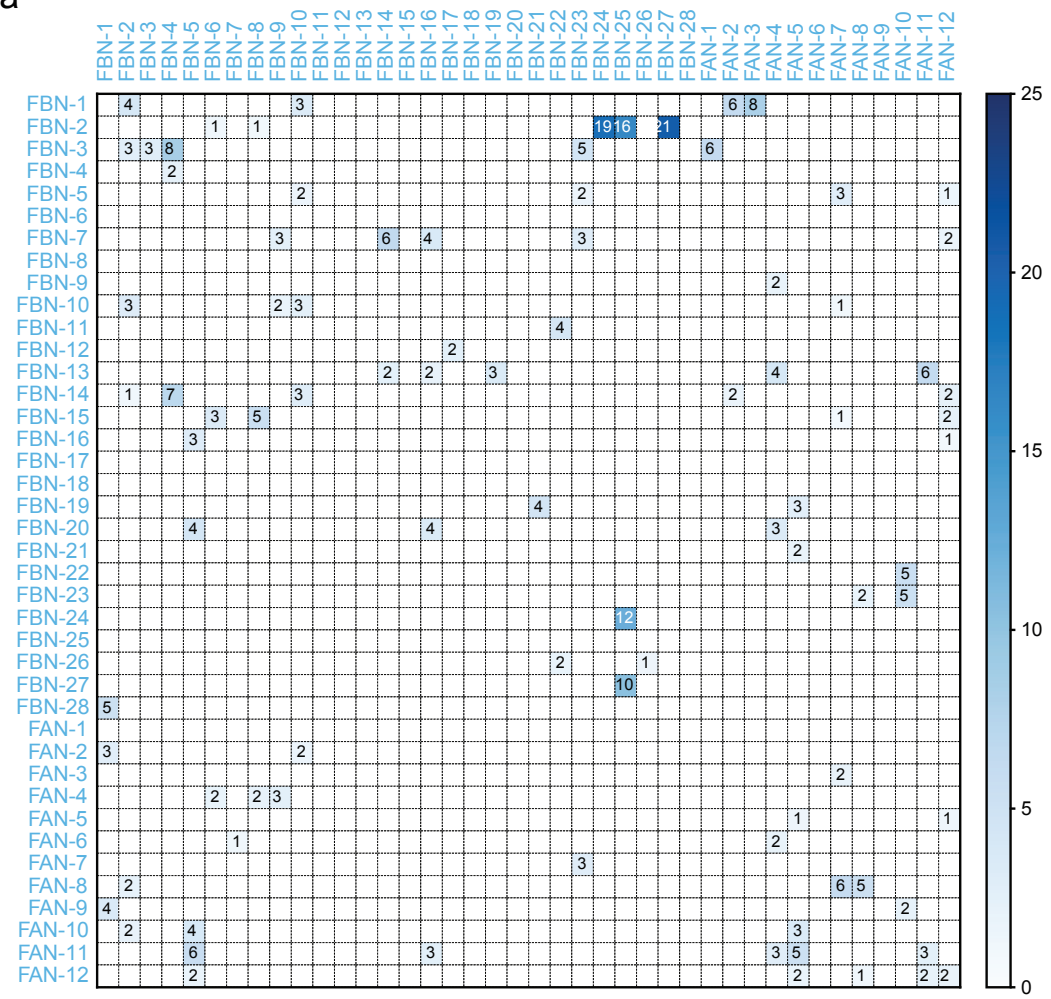

C

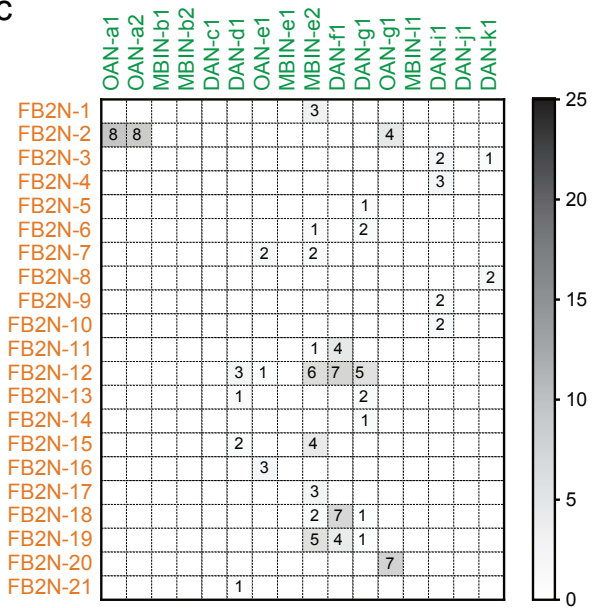

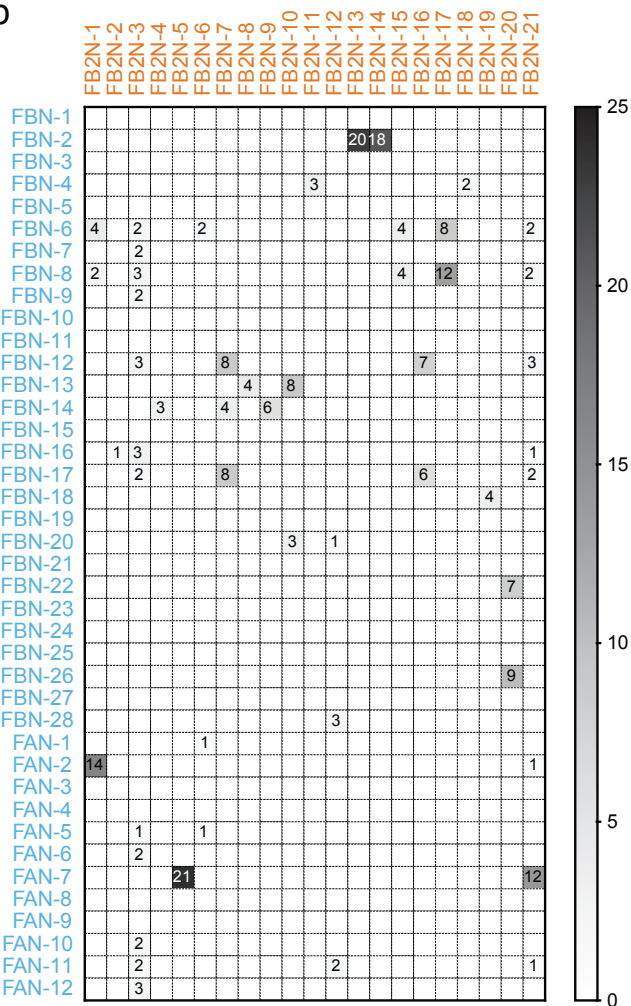

d

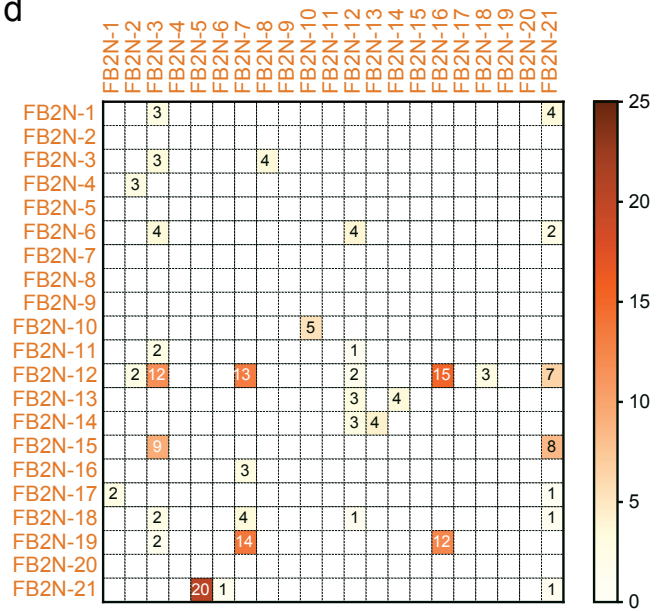

Extended Data Figure 13: (related to Fig. 5) Connectivity matrices of feedback neurons with each other and with modulatory neurons.

Connectivity matrix showing normalized synaptic input (expressed as \% input, computed as in Fig. 2b) each postsynaptic neuron (columns) receives from each presynaptic neuron (rows). Only reliable connections are shown for which both the left and right homologous connections have at least 3 synapses, and their sum is at least 10, and for which the postsynaptic neuron receives at least $1 \%$ of input from the presynaptic neuron.

a FBNs (rows) synapsing onto FBNs (columns). b FBNs (rows) synapsing onto FB2Ns (columns). c FB2Ns (rowss) synapsing onto FB2Ns (columns). d FB2Ns (rows) synapse onto modulatory neurons (columns). Modulatory neurons are grouped by their compartments and lobes. CA, Calyx; IP, Intermediate peduncle; LP, Lower peduncle; LA, Lateral appendix; UVL, Upper vertical lobe; IVL, Intermediate vertical lobe; LVL, Lower vertical lobe; SHA, Shaft; UT, Upper toe; IT, Intermediate toe; LT, Lower toe. 
EscbiloRxiv, prephintidei: hittpsog/doi.org/10.1101/649731; this version posted May 27, 2019. The copyright holder for this preprint (which was not certified by peer review) is the author/funder. All rights reserved. No reuse allowed without permission.

\section{Extended Data Figure 14}

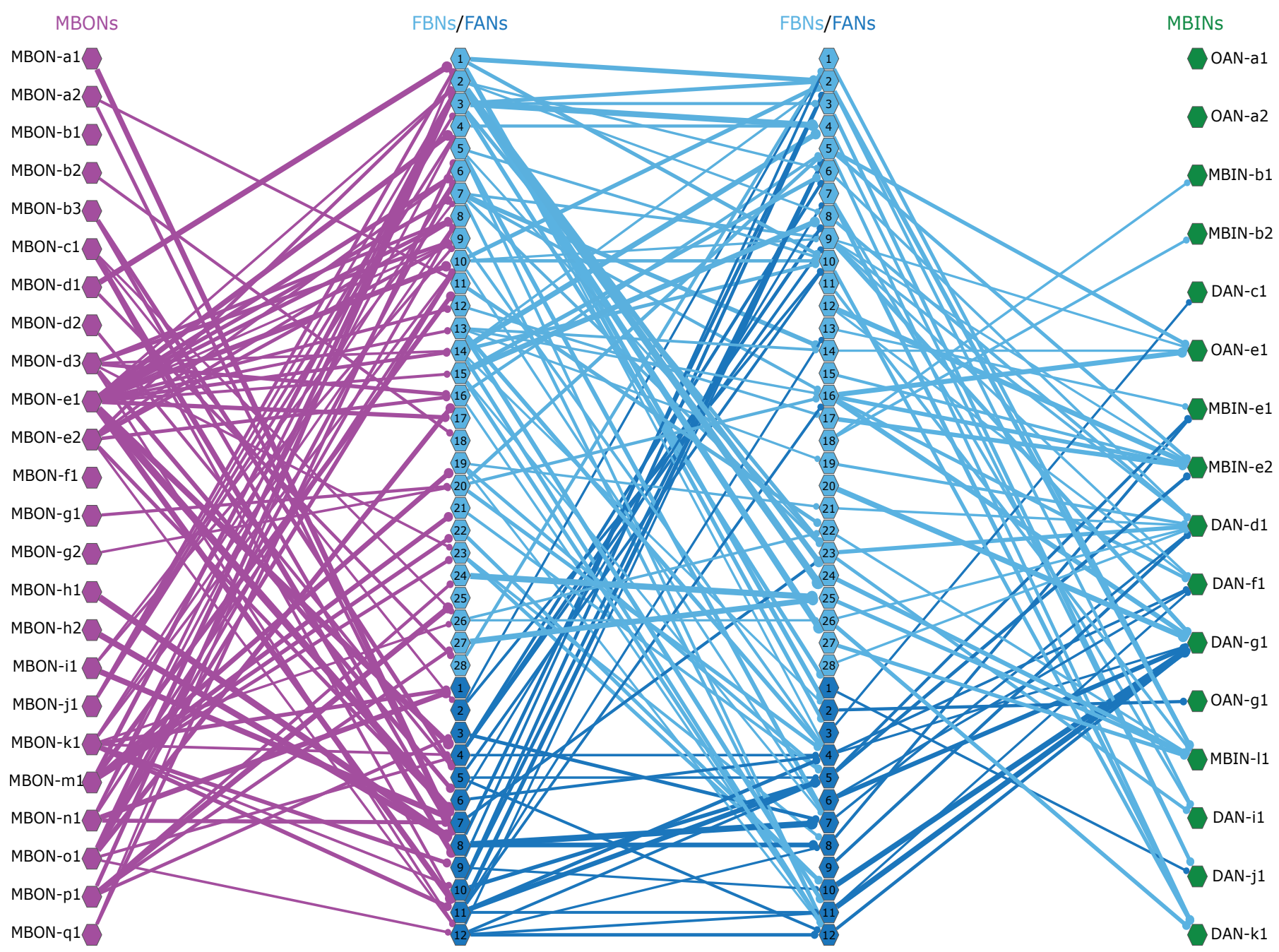

Extended Data Figure 14: (related to Fig. 5) Two-step feedback via FBNs. The interconnections among FBNs enable them to also provide two-step (in addition to one-step) feedback to modulatory neurons, similarly to FB2Ns (but the latter by definition do not receive direct inputs from MBONs). Left-right homologous neurons have been grouped as a type, and only connections with 10 or more synapses are shown. With this stringent connectivity criterium, almost all identified FBNs participate in two-step feedback motifs, except for FBN-12, FBN-13, FBN-15, FBN-18, FBN-20, FBN-27, FBN-28 and FAN-9, which do not receive inputs from other FBNs. All modulatory neurons receive two-step feedback via FBNs, except for OAN-a1 and OAN-a2. 